ANALYSIS \& PDE Volume $4 \quad$ No. $3 \quad 2011$

SEIM IBRAHIM, NADER MASMOUDI AND KENJI NAKANISHI

SCATTERING THRESHOLD FOR THE FOCUSING NONLINEAR KLEIN-GORDON EQUATION 


\title{
SCATTERING THRESHOLD FOR THE FOCUSING NONLINEAR KLEIN-GORDON EQUATION
}

\author{
Slim Ibrahim, NADER Masmoudi And Kenji NAKANishi
}

\begin{abstract}
We show scattering versus blow-up dichotomy below the ground state energy for the focusing nonlinear Klein-Gordon equation, in the spirit of Kenig and Merle for the $H^{1}$ critical wave and Schrödinger equations. Our result includes the $H^{1}$ critical case, where the threshold is given by the ground state for the massless equation, and the 2D square-exponential case, where the mass for the ground state may be modified, depending on the constant in the sharp Trudinger-Moser inequality. The main difficulty is the lack of scaling invariance in both the linear and the nonlinear terms.
\end{abstract}

1. Introduction

2. Variational characterizations

3. Blow-up

4. Global space-time norm

5. Profile decomposition

6. Extraction of a critical element

7. Extinction of the critical element

A table of notation can be found on page 458.

\section{Introduction}

The problem and overview. We study global and asymptotic behavior of solutions in the energy space for the nonlinear Klein-Gordon equation (NLKG):

$$
\ddot{u}-\Delta u+u=f^{\prime}(u), \quad u: \mathbb{R}^{1+d} \rightarrow \mathbb{R} \quad(d \in \mathbb{N}),
$$

where $f: \mathbb{R} \rightarrow \mathbb{R}$ is a given function. Typical examples that we can treat are the power nonlinearities in any dimension:

$$
f(u)=\lambda|u|^{p+2} \quad\left(2 \star<p+2 \leq 2^{\star}, \quad \lambda \geq 0\right),
$$

where $2 \star$ and $2^{\star}$ respectively denote the $L^{2}$ and $H^{1}$ critical powers

$$
2_{\star}=2+\frac{4}{d}, \quad 2^{\star}= \begin{cases}2+\frac{4}{d-2} & \text { if } d \geq 3, \\ \infty & \text { if } d \leq 2\end{cases}
$$

MSC2000: 35L70, 35B40, 35B44, 47J30.

Keywords: nonlinear Klein-Gordon equation, scattering theory, blow-up solution, ground state, Sobolev critical exponent, Trudinger-Moser inequality. 
and the square-exponential nonlinearity in two spatial dimensions:

$$
f(u)=\lambda|u|^{p} e^{\kappa|u|^{2}}, \quad(d=2, p>4, \lambda \geq 0, \kappa>0),
$$

which is related to the critical case for the Trudinger-Moser inequality. The equation conserves (at least formally) the energy

$$
E(u ; t)=E(u(t), \dot{u}(t)):=\int_{\mathbb{R}^{d}} \frac{|\dot{u}|^{2}+|\nabla u|^{2}+|u|^{2}}{2}-f(u) d x .
$$

The main goal in this paper is to give necessary and sufficient conditions for the solution $u$ to scatter, which means that $u$ is asymptotic to some free solutions as $t \rightarrow \pm \infty$, under the condition that $u$ has less energy than the least energy static solution, namely the ground state. In the defocusing case, where $f$ has the opposite sign, one has the scattering result for all finite energy solutions, see [Brenner 1984; Ginibre and Velo 1985a; Nakanishi 1999a; 1999b; 2001; Ibrahim et al. 2009]. In the focusing case, it turns out that the solutions below the ground energy split into the scattering solutions and the blow-up solutions (in both time directions in both cases). Such results have been recently established for many other equations including the nonlinear wave equation (NLW), the nonlinear Schrödinger equation (NLS), the YangMills system and the wave maps, since Kenig-Merle's [Kenig and Merle 2006] on NLS with the $H^{1}$ critical power (i.e. $p+2=2^{\star}$ in (1-2)); see [Akahori and Nawa 2010; Côte et al. 2008; Duyckaerts et al. 2008; Kenig and Merle 2008; Killip et al. 2008; 2009; Krieger and Schlag 2009; Sterbenz and Tataru 2010; Tao 2008a; 2008b; 2008c; 2009a; 2009b].

To be more precise, let us recall the result by Kenig and Merle for the critical nonlinear wave equation

$$
\ddot{u}-\Delta u=f^{\prime}(u), \quad f(u)=|u|^{2^{\star}} .
$$

Let $E^{(0)}(u)$ be the conserved energy, and $Q$ be a static solution with the least energy:

$$
E^{(0)}(u):=\int_{\mathbb{R}^{d}} \frac{|\dot{u}|^{2}+|\nabla u|^{2}}{2}+f(u) d x, \quad Q(x):=\left[1+\frac{|x|^{2}}{d(d-2)}\right]^{-(d-2) / 2} .
$$

Kenig and Merle [2008] proved that every solution with $E^{(0)}(u)<E^{(0)}(Q)$ scatters in the energy space as $t \rightarrow \pm \infty$, provided that $\|\nabla u(0)\|_{L^{2}}<\|\nabla Q\|_{L^{2}}$, and otherwise it blows up in finite time both for $t>0$ and for $t<0$. The idea of their proof is to bring the concentration compactness argument into the scattering problem by using space-time norms and the concept of a "critical element", that is, the minimal non-scattering solution.

The equations in those papers following Kenig and Merle have a common important property-the scaling invariance. It is further shared with the solution space (either the energy space or $L^{2}$, i.e. the critical case), except for the NLS with a subcritical power [Duyckaerts et al. 2008; Akahori and Nawa 2010]. The scaling invariance brings significant difficulties for the analysis, but also a lot of algebraic or geometric structures and simplifications. Hence it is a natural question what happens if the invariance is broken in the linear and the nonlinear parts of the equation. This is the main technical challenge in this paper.

The dichotomy into the global existence and the blow-up has been known long before the scattering result of Kenig and Merle, under the name of "potential well", defined by derivatives of the static energy 
functional. More precisely, Payne and Sattinger [1975] proved on bounded domains the dichotomy into blow-up and global existence for solutions below the ground energy, by the sign of the functional

$$
K_{1,0}(u):=\int|\nabla u|^{2}+|u|^{2}-u f^{\prime}(u) d x .
$$

It is easy to observe that their argument applies to the whole space $\mathbb{R}^{d}$ as soon as one has the local wellposedness in the energy space. Hence our primary task is to prove the scattering result in the region of global existence. Then our first problem due to the inhomogeneity is that the above functional $K_{1,0}$ is not suited for the scattering proof, though it is useful for the blow-up and global existence. More specifically, we want to use the functional

$$
K_{d,-2}(u):=\int 2|\nabla u|^{2}+d\left[u f^{\prime}(u)-2 f(u)\right] d x,
$$

which is related to the virial identity. There is actually a one-parameter family of functionals, corresponding to various scalings, each of which defines a splitting of the solutions below the ground energy by its sign. For example, Shatah [1985] used another functional

$$
K_{0,1}(u):=\int \frac{d-2}{2}|\nabla u|^{2}+\frac{d}{2}|u|^{2}-d f(u) d x,
$$

to prove the instability of the standing waves. Note that in his proof the instability is not given by blowup in the region $K_{0,1}(u)<0$. More recently, Ohta and Todorova [2007] proved blow-up in the region $K_{d,-2}(u)<0$, but they need radial symmetry for the powers $p$ close to $2^{\star}$.

The special feature of the critical wave Equation (1-6) is that those functionals are the same modulo constant multiples, which is exactly due to the scaling invariance. For the NLS with a subcritical power [Duyckaerts et al. 2008; Akahori and Nawa 2010], the functionals are different from each other, but the situation is much better than NLKG, because they contain only two terms (without the $L^{2}$ norm), the $L^{2}$ is another conserved quantity, and the virial identity is used both for the blow-up and for the scattering, while $K_{1,0}$ is not so useful for NLS.

It turns out, however, that those algebraically different functionals for NLKG define the same splitting below the threshold energy. This observation does not seem to be well recognized, but it is indeed crucial for the proof of the dichotomy, since we need different functionals for the blow-up and for the scattering.

One interesting feature resulting from the breakdown of the scaling is that, for some nonlinearity, the energy threshold is not given by the ground state of the original NLKG, but by that of a modified equation. More precisely, for the $H^{1}$ critical power $\left(p+2=2^{\star}\right)$ in three dimensions or higher, the threshold is given by that of the critical wave equation, or massless Klein-Gordon equation. This can be expected because the concentration by the critical scaling makes the $L^{2}$ norm vanish while preserving other components, namely the massless energy. However the transition from the Klein-Gordon to the wave requires some effort in the scattering proof.

We find another instance of mass modification, which is more surprising. That is in two dimensions and for nonlinearities which grow slightly slower than the square exponential $e^{|u|^{2}}$, where the mass for the threshold ground energy can change to any number between 0 and 1 , depending on the constant in the sharp $\left(L^{2}\right)$ Trudinger-Moser inequality. Thus we prove the existence of extremizers as well as the ground states with mass less than or equal to the sharp constant, which also seems new for general 
nonlinearity on the whole plane. For the existence of the ground state on bounded domains, we refer to [Figueiredo et al. 1995; Adimurthi 1990; Adimurthi and Struwe 2000]. One should be warned, however, that the situation on the whole plane is different from that on disks, unlike the higher dimensional Sobolev critical case, since here the concentration compactness has to be accompanied with a leak of $L^{2}$ norm to the spatial infinity. This will be discussed in [Ibrahim et al. 2011].

It is worth noting that the scattering result in the focusing exponential case is actually easier to obtain than in the defocusing case, concerning the global Strichartz estimate. This is because the (mass-modified) ground energy threshold implies that our solutions are in the subcritical regime for the Trudinger-Moser inequality. Hence concentration of energy is a priori precluded, and so we do not need the concentration radius or the localized Strichartz estimate used in [Ibrahim et al. 2009] on the Trudinger-Moser threshold in the defocusing case. This is another striking difference from the power case, where the analysis for the focusing case essentially contains that for the defocusing case.

Main result. To state the main results of this paper, we need to introduce some notation and assumptions for the variational setting and the nonlinear setting of the problem.

Variational setting. To specify our class of solutions, we need the static energy

$$
J(\varphi):=\frac{1}{2} \int_{\mathbb{R}^{d}}\left[|\nabla \varphi|^{2}+|\varphi|^{2}\right] d x-F(\varphi), \quad F(\varphi):=\int_{\mathbb{R}^{d}} f(\varphi) d x,
$$

and its derivatives with respect to different scalings. In the critical and exponential cases, we also need the energy with a modified mass $c \geq 0$,

$$
J^{(c)}(\varphi)=\frac{1}{2} \int_{\mathbb{R}^{d}}\left[|\nabla \varphi|^{2}+c|\varphi|^{2}\right] d x-F(\varphi) .
$$

For any $\alpha, \beta, \lambda \in \mathbb{R}$ and $\varphi: \mathbb{R}^{d} \rightarrow \mathbb{R}$, we define the two-parameter rescaling family

$$
\varphi_{\alpha, \beta}^{\lambda}(x)=e^{\alpha \lambda} \varphi\left(e^{-\beta \lambda} x\right),
$$

and the differential operator $\mathscr{L}_{\alpha, \beta}$ acting on any functional $S: H^{1}\left(\mathbb{R}^{d}\right) \rightarrow \mathbb{R}$ by

$$
\mathscr{L}_{\alpha, \beta} S(\varphi)=\left.\frac{d}{d \lambda}\right|_{\lambda=0} S\left(\varphi_{\alpha, \beta}^{\lambda}\right) .
$$

The scaling derivative of the static energy is denoted by

$$
\begin{aligned}
K_{\alpha, \beta}(\varphi) & :=\mathscr{L}_{\alpha, \beta} J(\varphi) \\
& =\int_{\mathbb{R}^{d}}\left[\frac{2 \alpha+(d-2) \beta}{2}|\nabla \varphi|^{2}+\frac{2 \alpha+d \beta}{2}|\varphi|^{2}-\alpha \varphi f^{\prime}(\varphi)-d \beta f(\varphi)\right] d x, \\
K_{\alpha, \beta}^{(c)}(\varphi) & :=\mathscr{L}_{\alpha, \beta} J^{(c)}(\varphi) .
\end{aligned}
$$

For each $(\alpha, \beta) \in \mathbb{R}^{2}$ in the range

$$
\alpha \geq 0, \quad 2 \alpha+d \beta \geq 0, \quad 2 \alpha+(d-2) \beta \geq 0, \quad(\alpha, \beta) \neq(0,0),
$$


we consider the constrained minimization problem

$$
m_{\alpha, \beta}=\inf \left\{J(\varphi) \mid \varphi \in H^{1}\left(\mathbb{R}^{d}\right), \varphi \neq 0, K_{\alpha, \beta}(\varphi)=0\right\} .
$$

We will prove that it is attained, (after a modification of the mass in some cases), provided that $(\alpha, \beta)$ is in the above range (1-16). The condition on $(\alpha, \beta)$ is also necessary in general (see Proposition A.1).

Our solutions start from the following subsets of the energy space:

$$
\begin{aligned}
& \mathcal{K}_{\alpha, \beta}^{+}=\left\{\left(u_{0}, u_{1}\right) \in H^{1}\left(\mathbb{R}^{d}\right) \times L^{2}\left(\mathbb{R}^{d}\right) \mid E\left(u_{0}, u_{1}\right)<m_{\alpha, \beta}, K_{\alpha, \beta}\left(u_{0}\right) \geq 0\right\}, \\
& \mathcal{K}_{\alpha, \beta}^{-}=\left\{\left(u_{0}, u_{1}\right) \in H^{1}\left(\mathbb{R}^{d}\right) \times L^{2}\left(\mathbb{R}^{d}\right) \mid E\left(u_{0}, u_{1}\right)<m_{\alpha, \beta}, K_{\alpha, \beta}\left(u_{0}\right)<0\right\} .
\end{aligned}
$$

Nonlinear setting. For the nonlinearity $f$, we consider the following three cases: the $H^{1}$ subcritical $(d \geq 1)$, the $2 \mathrm{D}$ exponential case, and the $H^{1}$ critical $(d \geq 3)$ cases. First we assume that $f: \mathbb{R} \rightarrow \mathbb{R}$ is $C^{2}$ and

$$
f(0)=f^{\prime}(0)=f^{\prime \prime}(0)=0 .
$$

Secondly for the variational arguments, we need some monotonicity and convexity conditions. Let $D$ denote the linear operator defined by

$$
D f(u):=u f^{\prime}(u) .
$$

We assume that $f$ satisfies for some $\varepsilon>0$,

$$
(D-2 \star-\varepsilon) f \geq 0, \quad(D-2)(D-2 \star-\varepsilon) f \geq 0,
$$

which implies in particular that

$$
D^{2} f \geq\left(2_{\star}+\varepsilon\right) D f \geq\left(2_{\star}+\varepsilon\right)^{2} f \geq 0 .
$$

Finally we need regularity and growth conditions, which can differ for small $|u|$ and large $|u|$. Fix a cut-off function $\chi \in C_{0}^{\infty}(\mathbb{R})$ satisfying $\chi(r)=1$ for $|r| \leq 1$ and $\chi(r)=0$ for $|r| \geq 2$, and set

$$
\chi_{R}(x):=\chi(|x| / R)
$$

for arbitrary vector $x$ and $R>0$. Decompose the nonlinearity by

$$
f_{S}(u):=\chi_{1}(u) f(u), \quad f_{L}(u)=f(u)-f_{S}(u) .
$$

We assume that for some $p_{1}>2 \star-2$

$$
\begin{cases}\left|f_{S}^{\prime \prime}(u)\right| \lesssim|u|^{p_{1}} & (d \leq 4) \\ \left|f_{S}^{\prime \prime}\left(u_{1}\right)-f_{S}^{\prime \prime}\left(u_{2}\right)\right| \lesssim\left|u_{1}-u_{2}\right|^{p_{1}} & (d \geq 5)\end{cases}
$$

where we should choose $p_{1}<1$ for $d \geq 5$.

For the behavior of $f$ for large $|u|$, we distinguish three cases:

(1) $H^{1}$ subcritical case: We assume that for some $p_{2}<2^{\star}-2$

$$
\begin{cases}\left|f_{L}^{\prime \prime}(u)\right| \lesssim|u|^{p_{2}} & (2 \leq d \leq 4) \\ \left|f_{L}^{\prime \prime}\left(u_{1}\right)-f_{L}^{\prime \prime}\left(u_{2}\right)\right| \lesssim\left(\left|u_{1}\right|+\left|u_{2}\right|\right)^{p_{2}-1}\left|u_{1}-u_{2}\right| & \left(d \geq 5 \text { and } p_{2} \geq 1\right) \\ \left|f_{L}^{\prime \prime}\left(u_{1}\right)-f_{L}^{\prime \prime}\left(u_{2}\right)\right| \lesssim\left|u_{1}-u_{2}\right|^{p_{2}} & \left(d \geq 5 \text { and } p_{2}<1\right) .\end{cases}
$$


We allow $p_{2}=2^{\star}-2$ in some of the later arguments. There is no growth restriction for $d=1$. A typical example is

$$
f(u)=\lambda_{1}|u|^{q_{1}}+\cdots \lambda_{k}|u|^{q_{k}},
$$

where $\lambda_{j}>0$ and $2 \star<q_{j}<2^{\star}$ for all $j$, which satisfies (1-26) as well as (1-19), (1-21) and (1-25).

(2) $H^{1}$ critical case. We assume

$$
d \geq 3, \quad f(u)=|u|^{2^{\star}} / 2^{\star} .
$$

In this case, we do not include lower powers in order to avoid their nontrivial effects in the variational characterization. The absence of lower powers will only be used in Section 2. In particular the Strichartz spaces we use in Section 4 can handle the sum of a critical power with a subcritical function.

(3) $2 \mathrm{D}$ exponential case: We assume that

$$
\begin{aligned}
& d=2 \\
& \exists \kappa_{0} \geq 0\left\{\begin{array}{l}
\forall \kappa>\kappa_{0}, \lim _{|u| \rightarrow \infty} f_{L}^{\prime \prime}(u) e^{-\kappa|u|^{2}}=0 \\
\forall \kappa<\kappa_{0}, \lim _{|u| \rightarrow \infty} f_{L}(u) e^{-\kappa|u|^{2}}=\infty
\end{array}\right\} \text { and if } \kappa_{0}>0 \text { then } \lim _{|u| \rightarrow \infty} \frac{f_{L}(u)}{D f_{L}(u)}=0 .
\end{aligned}
$$

Then we define $C_{\mathrm{TM}}^{\star}$ by

$$
C_{\mathrm{TM}}^{\star}(F)=\sup \left\{2 F(\varphi)\|\varphi\|_{L^{2}\left(\mathbb{R}^{2}\right)}^{-2} \mid 0 \neq \varphi \in H^{1}\left(\mathbb{R}^{2}\right), \kappa_{0}\|\nabla \varphi\|_{L^{2}\left(\mathbb{R}^{2}\right)}^{2} \leq 4 \pi\right\} .
$$

For example, all the conditions are satisfied by

$$
f(u)=e^{\kappa_{0}|u|^{2}}-1-\kappa_{0}|u|^{2}-\frac{1}{2} \kappa_{0}^{2}|u|^{4}
$$

and by

$$
f(u)=|u|^{p} e^{\kappa_{0}|u|^{2}+\gamma|u|},
$$

where $p>4, \kappa_{0} \geq 0$, and $\max (-\gamma, 0) \ll 1$ (depending on $\kappa_{0}(p-4)$ ). More specifically, it suffices to have for all $u \in[0, \infty)$ that

$$
8 \kappa_{0} u^{2}+3 \gamma u+2(p-4)>0
$$

since, putting $g:=D f / f=2 \kappa_{0} u^{2}+\gamma u+p$, we have $2 \star=4$ and

$$
\begin{aligned}
(D-2)(D-4) f & =\left[(g-4)^{2}+D g+2(g-4)\right] f \\
D g+2(g-4) & =8 \kappa_{0} u^{2}+3 \gamma u+2(p-4)=2[g(3 u / 2)-4]-u^{2} / 2 .
\end{aligned}
$$

In addition, one can easily observe that $C_{\mathrm{TM}}^{\star}(F)=\infty$ if $\gamma \geq 0$ and $C_{\mathrm{TM}}^{\star}(F)<\infty$ if $\gamma<0$, using Moser's sequence of functions for the former, and by the spherical symmetrization for the latter (compare [Moser 1971; Adachi and Tanaka 2000; Ruf 2005]). ${ }^{1}$

${ }^{1}$ Actually, the optimal (fastest) growth to have $C_{\mathrm{TM}}^{\star}(F)<\infty$ is given by

$$
f(u) \sim e^{\kappa_{0}|u|^{2}} /|u|^{2} \quad(|u| \rightarrow \infty),
$$

as shown in [Ibrahim et al. 2011]. The results in the present paper do not rely on this observation, though it seems to have its own interest. 
In short, our assumption on $f$ is that

$$
(1-19),(1-21),(1-25) \text {, and [(1-26) or (1-28) or (1-29)]. }
$$

Then by Sobolev or Trudinger-Moser, we observe that $F, \mathscr{L}_{\alpha, \beta} F$ and $\mathscr{L}_{\alpha, \beta}^{2} F$ are continuous functionals on $H^{1}\left(\mathbb{R}^{d}\right)$.

Now we can state our main result. Denote the quadratic part of the energy (i.e., the linear energy) by

$$
E^{Q}(u ; t)=E^{Q}(u(t), \dot{u}(t)):=\int_{\mathbb{R}^{d}} \frac{|\dot{u}|^{2}+|\nabla u|^{2}+|u|^{2}}{2} d x .
$$

Theorem 1.1. Assume (1-36) for $f$. Then for all $(\alpha, \beta)$ in (1-16), both $m_{\alpha, \beta}$ and $\mathscr{K}_{\alpha, \beta}^{ \pm}$are independent of $(\alpha, \beta)$. Moreover (1-1) is locally wellposed in the energy space $H^{1} \times L^{2}$, and

(1) If $(u(0), \dot{u}(0)) \in \mathcal{K}_{\alpha, \beta}^{-}$, then $u$ extends neither for $t \rightarrow \infty$ nor for $t \rightarrow-\infty$ as the unique strong solution in $H^{1} \times L^{2}$.

(2) If $(u(0), \dot{u}(0)) \in \mathscr{K}_{\alpha, \beta}^{+}$, then $u$ scatters both in $t \rightarrow \pm \infty$ in the energy space. In other words, $u$ is a global solution and there are $v_{ \pm}$satisfying

$$
\begin{gathered}
\ddot{v}_{ \pm}-\Delta v_{ \pm}+v_{ \pm}=0, \\
E^{Q}\left(u-v_{ \pm}, \dot{u}-\dot{v}_{ \pm}\right) \rightarrow 0 \quad(t \rightarrow \pm \infty) .
\end{gathered}
$$

The dichotomy of global existence versus blow-up in the subcritical case was essentially given in [Payne and Sattinger 1975], using $K_{1,0}$, on bounded domains. Hence our main contribution is the scattering part, and the parameter independence of $\mathscr{K}_{\alpha, \beta}^{ \pm}$. The corresponding result in the defocusing case (hence only the scattering) has been shown in [Brenner 1984; Ginibre and Velo 1985b] for the subcritical $f$ in three dimensions and higher, in [Nakanishi 1999b] in lower dimensions, in [Nakanishi 1999a] for the $H^{1}$ critical $f$, and in [Ibrahim et al. 2009] for the 2D exponential nonlinearity. The massless $H^{1}$ critical case (the other powers cannot be controlled by the massless energy) was solved by [Bahouri and Shatah 1998; Bahouri and Gérard 1999] for the defocusing $f$ and by [Kenig and Merle 2008] for the focusing nonlinearity.

The parameter independence of $m_{\alpha, \beta}$ seems to be known in the study of stability of standing waves, but the authors could not find an available result as general as the above one. See [Ohta and Todorova 2007; Zhang 2002] for partial results. We quote a recent paper [Jeanjean and Le Coz 2009] for a pure power nonlinearity, but unfortunately their range of $(\alpha, \beta)$ was not correct (the condition $\alpha \geq 0$ was overlooked; its necessity is shown by Proposition A.1).

The parameter independence of $\mathscr{K}_{\alpha, \beta}^{ \pm}$, on the other hand, does not seem to have got much attention from the stability analysis, but it is essential in our proof of the scattering, since the monotonicity is given for the blow-up and for the scattering in terms of different $K_{\alpha, \beta}$, respectively $K_{1,0}$ and $K_{d,-2}$.

Thanks to the parameter independence, we may write

$$
m=m_{\alpha, \beta} \quad \text { and } \quad \mathscr{K}^{ \pm}=\mathscr{K}_{\alpha, \beta}^{ \pm}
$$

We will also show the following important properties of the energy threshold. 
Proposition 1.2. Let the assumptions be as in Theorem 1.1.

(1) In the subcritical case (1-26), the threshold energy $m$ is attained by some $Q \in H^{1}\left(\mathbb{R}^{d}\right)$, independent of $(\alpha, \beta)$, solving the static equation

$$
-\Delta Q+Q=f^{\prime}(Q)
$$

with the least energy $J(Q)=m$ among the solutions in $H^{1}\left(\mathbb{R}^{d}\right)$. In other words, $m$ is attained by the ground states.

(2) In the critical case (1-28), there is no minimizer for (1-17), but we have

$$
m=J^{(0)}(Q)
$$

for a static solution $Q \in \dot{H}^{1}\left(\mathbb{R}^{d}\right)$ of the massless equation

$$
-\Delta Q=f^{\prime}(Q)
$$

with the least massless energy $J^{(0)}$. In other words, m equals the massless ground energy.

(3) In the exponential case (1-29), let

$$
c:=\min \left(1, C_{\mathrm{TM}}^{\star}(F)\right),
$$

where $C_{\mathrm{TM}}^{\star}(F)$ is as in (1-30). Then

$$
m=J^{(c)}(Q)
$$

for a static solution $Q \in H^{1}\left(\mathbb{R}^{2}\right)$ of the mass-modified equation

$$
-\Delta Q+c Q=f^{\prime}(Q)
$$

with the least energy $J^{(c)}(Q)$. Moreover we have

$$
m \leq 2 \pi / \kappa_{0},
$$

where the equality holds if and only if $C_{\mathrm{TM}}^{\star}(F) \leq 1$, and $m=m_{\alpha, \beta}$ is attained in (1-17) if and only if $C_{\mathrm{TM}}^{\star}(F) \geq 1$.

Again this is well known in the subcritical case. Hence the main novelty is in the mass change in the critical and exponential cases. Note that the ground state $Q$ with a different mass $c \in[0,1)$ yields standing wave solutions $e^{ \pm i t \omega} Q(x)$ with $1-\omega^{2}=c$. But it is not a true obstruction for the scattering, because its dynamical energy is above $m$, although $m$ is the right threshold in the sense that for higher energy level $E>m$ the sets $\mathscr{K}^{ \pm}$are no longer separated from each other, that is, $\partial \mathscr{K}^{+} \cap \partial \mathcal{K}^{-} \neq \varnothing$.

Some notation. We recall some standard notation. F denotes the Fourier transform on $\mathbb{R}^{d}$, and

$$
\langle\nabla\rangle:=\sqrt{1-\Delta}=\mathscr{F}^{-1} \sqrt{1+\mid \xi^{2}} \mathscr{F} .
$$


$L^{p}, H^{s}, B_{p, q}^{s}$ and $\dot{B}_{p, q}^{s}$ respectively denote the Lebesgue, Sobolev, inhomogeneous and homogeneous Besov spaces on $\mathbb{R}^{d}$. For later use we recall the most used functionals $K_{\alpha, \beta}$ and $H_{\alpha, \beta}$ :

$$
\begin{aligned}
K_{1,0}(\varphi) & =\int_{\mathbb{R}^{d}}\left[|\nabla \varphi|^{2}+|\varphi|^{2}-\varphi f^{\prime}(\varphi)\right] d x, \\
K_{0,1}(\varphi) & =\int_{\mathbb{R}^{d}}\left[\frac{d-2}{2}|\nabla \varphi|^{2}+\frac{d}{2}|\varphi|^{2}-d f(\varphi)\right] d x, \\
K_{d,-2}(\varphi) & =\int_{\mathbb{R}^{d}}\left[2|\nabla \varphi|^{2}-d(D-2) f(\varphi)\right] d x, \\
H_{1,0}(\varphi) & =\frac{1}{2} \int_{\mathbb{R}^{d}}[(D-2) f(\varphi)] d x, \\
H_{0,1}(\varphi) & =\int_{\mathbb{R}^{d}}\left[\frac{1}{d}|\nabla \varphi|^{2}\right] d x, \\
H_{d,-2}(\varphi) & =\int_{\mathbb{R}^{d}}\left[\frac{1}{2}|\varphi|^{2}+\frac{d}{4}\left(D-2_{*}\right) f(\varphi)\right] d x .
\end{aligned}
$$

We give a table of notation on page 458 .

\section{Variational characterizations}

In this section, we prove Proposition 1.2. In particular we prove the existence of ground states as constrained minimizers, the $(\alpha, \beta)$-independence of the splittings, together with various estimates for solutions below the threshold by variational arguments, which will be used for the scattering and blowup.

Throughout this section, we assume that $(\alpha, \beta)$ is in the range (1-16). For ease of presentation, we often omit $(\alpha, \beta)$ from the subscript. We associate with it the following two numbers:

$$
\bar{\mu}=\max (2 \alpha+d \beta, 2 \alpha+(d-2) \beta), \quad \underline{\mu}=\min (2 \alpha+d \beta, 2 \alpha+(d-2) \beta),
$$

which come from the scaling exponents for $\dot{H}^{1}$ and $L^{2}$ in (1-13). Notice that in the range (1-16), we have $\bar{\mu}>0, \underline{\mu} \geq 0$, and that $\alpha=\underline{\mu}=0$ if and only if $(d, \alpha)=(2,0)$, which will often be an exceptional case in the following arguments.

We decompose $K_{\alpha, \beta}=\mathscr{L}_{\alpha, \beta} J$ into the quadratic and the nonlinear parts:

$$
K_{\alpha, \beta}=K_{\alpha, \beta}^{Q}+K_{\alpha, \beta}^{N}, \quad K_{\alpha, \beta}^{Q}(\varphi)=\mathscr{L}_{\alpha, \beta}\|\varphi\|_{H^{1}}^{2} / 2, \quad K_{\alpha, \beta}^{N}(\varphi)=-\mathscr{L}_{\alpha, \beta} F(\varphi) .
$$

Then $K_{\alpha, \beta}^{Q}\left(\varphi_{\alpha, \beta}^{\lambda}\right)$ is non-negative and non-decreasing with respect to $\lambda \in \mathbb{R}$, and

$$
\lim _{\lambda \rightarrow-\infty} K_{\alpha, \beta}^{Q}\left(\varphi_{\alpha, \beta}^{\lambda}\right)=0
$$

from its explicit form.

Energy landscape in various scales. First we investigate how $J$ and its derivatives behave with respect to the scaling $\varphi_{\alpha, \beta}^{\lambda}$, in order to get $m_{\alpha, \beta}$ as a minimax value. The results of this subsection are essentially known, at least under more restrictions on the nonlinearity and $(\alpha, \beta)$.

We start from the origin of the energy space. 
Lemma 2.1 (Positivity of $K$ near 0 ). Assume that $f$ satisfies (1-36), and that $(\alpha, \beta)$ satisfies (1-16) and $(d, \alpha) \neq(2,0)$. Then for any bounded sequence $\varphi_{n} \in H^{1}\left(\mathbb{R}^{d}\right) \backslash\{0\}$ such that $K_{\alpha, \beta}^{Q}\left(\varphi_{n}\right) \rightarrow 0$, we have, for large $n$,

$$
K_{\alpha, \beta}\left(\varphi_{n}\right)>0 .
$$

Note that if $(d, \alpha)=(2,0)$ the conclusion is false, since in that case $K^{Q}\left(\varphi^{\lambda}\right)=e^{d \beta \lambda} K^{Q}(\varphi) \rightarrow 0$ as $\lambda \rightarrow-\infty$, but $K\left(\varphi^{\lambda}\right)=e^{d \beta \lambda} K(\varphi)$ can be negative.

Proof. First we consider the $H^{1}$ subcritical/critical cases. If $d \geq 2$ then

$$
|D f(\varphi)|+|f(\varphi)| \lesssim|\varphi|^{p_{1}+2}+|\varphi|^{p_{2}+2},
$$

for some $2 \star<p_{1}+2<p_{2}+2 \leq 2^{\star}$; hence, by the Gagliardo-Nirenberg inequality

$$
\|\varphi\|_{L_{x}^{q}}^{q} \lesssim\|\nabla \varphi\|_{L_{x}^{2}}^{d(q / 2-1)}\|\varphi\|_{L_{x}^{2}}^{d-q(d-2) / 2} \quad\left(2 \leq q \leq 2^{\star}\right)
$$

we obtain

$$
|F(\varphi)|+|\mathscr{L} F(\varphi)| \lesssim \sum_{q=p_{1}+2, p_{2}+2}\|\nabla \varphi\|_{L_{x}^{2}}^{d(q / 2-1)}\|\varphi\|_{L_{x}^{2}}^{d-q(d-2) / 2} .
$$

If $d=1$ then we can dispose of $f_{L}$ by Sobolev $H^{1}(\mathbb{R}) \subset L^{\infty}(\mathbb{R})$. Then we get

$$
|F(\varphi)|+|\mathscr{L} F(\varphi)| \lesssim\|\nabla \varphi\|_{L_{x}^{2}}^{p_{1} / 2+1}\|\varphi\|_{L_{x}^{2}}^{p_{1} / 2+1} C\left(\|\varphi\|_{H^{1}}\right),
$$

for some function $C$ determined by $f_{L}$.

Hence if $2 \alpha+(d-2) \beta>0$ then for any $d$ we have

$$
\left|K^{N}(\varphi)\right|=o\left(\|\nabla \varphi\|_{L_{x}^{2}}^{2}\right)=o\left(K^{Q}(\varphi)\right) .
$$

Under the assumption, $2 \alpha+(d-2) \beta=0$ is possible only for $d=1$; then, using (2-8),

$$
\left|K^{N}(\varphi)\right|=o\left(\|\varphi\|_{L_{x}^{2}}^{2}\right)=o\left(K^{Q}(\varphi)\right) .
$$

Finally we consider the 2D exponential case (1-29). Then we have

$$
|D f(\varphi)|+|f(\varphi)| \lesssim|\varphi|^{p}\left(e^{\kappa|\varphi|^{2}}-1\right),
$$

for some $p>2$ and any $\kappa>\kappa_{0}$. Since $\alpha>0$, we have $K^{Q}\left(\varphi_{n}\right) \gtrsim\left\|\nabla \varphi_{n}\right\|_{L^{2}}^{2} \rightarrow 0$, so it suffices to consider $\varphi \in H^{1}$ satisfying, for some $q>1$ such that $(4-p) q<2$,

$$
q \kappa\|\nabla \varphi\|_{L^{2}}^{2} \leq 2 \pi \text {. }
$$

Let $q^{\prime}=q /(q-1)$ be the Hölder conjugate. Then by Hölder, Gagliardo-Nirenberg (2-6) and the Trudinger-Moser inequality

$$
\|\nabla \varphi\|_{L^{2}\left(\mathbb{R}^{2}\right)}<\sqrt{4 \pi} \Longrightarrow \int_{\mathbb{R}^{2}}\left(e^{|\varphi|^{2}}-1\right) d x \lesssim \frac{\|\varphi\|_{L^{2}\left(\mathbb{R}^{2}\right)}^{2}}{4 \pi-\|\nabla \varphi\|_{L^{2}\left(\mathbb{R}^{2}\right)}^{2}},
$$

we obtain 


$$
\begin{aligned}
|\mathscr{L} F(\varphi)|+|F(\varphi)| & \lesssim\|\varphi\|_{L^{p q^{\prime}}}^{p}\left\|e^{q \kappa|\varphi|^{2}}-1\right\|_{L^{1}}^{1 / q} \lesssim\|\varphi\|_{L^{2}}^{2 / q^{\prime}}\|\nabla \varphi\|_{L^{2}}^{p-2 / q^{\prime}}\left[\frac{\|\varphi\|_{L^{2}}^{2}}{4 \pi-q \kappa\|\nabla \varphi\|_{L^{2}}^{2}}\right]^{1 / q} \\
& \lesssim\|\varphi\|_{L^{2}}^{2}\|\nabla \varphi\|_{L^{2}}^{p-2 / q^{\prime}}
\end{aligned}
$$

Since $p-2 / q^{\prime}>2$ by the choice of $q$, we get

$$
\left|K^{N}(\varphi)\right|=o\left(\|\nabla \varphi\|_{L^{2}}^{2}\right)=o\left(K^{Q}(\varphi)\right) .
$$

Thus in all cases $K(\varphi) \sim K^{Q}(\varphi)>0$ when $0<K^{Q}(\varphi) \ll 1$.

The following inequalities describe the graph of $J$, and will play the central role in the succeeding arguments.

Lemma 2.2 (Mountain-pass structure). Assume that $f$ satisfies (1-36) and $(\alpha, \beta)$ satisfies (1-16). Then for any $\varphi \in H^{1}\left(\mathbb{R}^{d}\right)$ we have

$$
\begin{aligned}
\left(\mathscr{L}_{\alpha, \beta}-\bar{\mu}\right)\|\varphi\|_{H^{1}}^{2} & \leq-2|\beta| \min \left(\|\varphi\|_{L^{2}}^{2},\|\nabla \varphi\|_{L^{2}}^{2}\right) \\
\left(\mathscr{L}_{\alpha, \beta}-\bar{\mu}\right) F(\varphi) & \geq \alpha \varepsilon F(\varphi)
\end{aligned}
$$

where $\varepsilon>0$ is given in (1-21). Hence

$$
\left(\bar{\mu}-\mathscr{L}_{\alpha, \beta}\right) J(\varphi) \geq \alpha \varepsilon F(\varphi)+|\beta| \min \left(\|\varphi\|_{L^{2}}^{2},\|\nabla \varphi\|_{L^{2}}^{2}\right) .
$$

Moreover we have

$$
-\left(\mathscr{L}_{\alpha, \beta}-\bar{\mu}\right)\left(\mathscr{L}_{\alpha, \beta}-\underline{\mu}\right) J(\varphi)=\left(\mathscr{L}_{\alpha, \beta}-\bar{\mu}\right)\left(\mathscr{L}_{\alpha, \beta}-\underline{\mu}\right) F(\varphi) \geq \frac{2 \alpha \varepsilon}{d+1} \mathscr{L}_{\alpha, \beta} F(\varphi) \geq \frac{2 \alpha \varepsilon \bar{\mu}}{d+1} F(\varphi) .
$$

Proof. First we observe that

$$
(\mathscr{L}-2 \alpha-(d-2) \beta)\|\nabla \varphi\|_{L_{x}^{2}}^{2}=0, \quad(\mathscr{L}-2 \alpha-d \beta)\|\varphi\|_{L_{x}^{2}}^{2}=0,
$$

and for any functional $S$ of the form $S(\varphi)=\int_{\mathbb{R}^{d}} S(\varphi) d x$,

$$
\mathscr{L}_{\alpha, \beta} S(\varphi)=\int_{\mathbb{R}^{d}}[(\alpha D+\beta d) s](\varphi) d x,
$$

where $D f(\varphi)=\varphi f^{\prime}(\varphi)$ as defined in (1-20). Using this, we obtain

$$
(\mathscr{L}-\bar{\mu})\|\varphi\|_{H^{1}}^{2}=-2|\beta| \times \begin{cases}\|\nabla \varphi\|_{L^{2}}^{2} & (\beta \geq 0) \\ \|\varphi\|_{L^{2}}^{2} & (\beta \leq 0)\end{cases}
$$

and also

$$
\mathscr{L} F(\varphi)=\int[\alpha(D-2)+2 \alpha+d \beta] f(\varphi) d x=\int[(\alpha D-2 \alpha+2 \beta)+2 \alpha+(d-2) \beta] f(\varphi) d x
$$

Since

using (1-21), we obtain

$$
\alpha D-2 \alpha+2 \beta=\alpha\left(D-2_{\star}\right)+\frac{2}{d}(2 \alpha+d \beta),
$$

$$
\mathscr{L} F \geq(\bar{\mu}+\alpha \varepsilon) F
$$


Using these computations, we have

$$
\begin{aligned}
-(\mathscr{L}-\bar{\mu})(\mathscr{L}-\underline{\mu}) J(\varphi) & =(\mathscr{L}-\bar{\mu})(\mathscr{L}-\underline{\mu}) F(\varphi) \\
& =\alpha \int(\alpha D-2 \alpha+2 \beta)(D-2) f(\varphi) d x \\
& \geq \alpha \varepsilon \int\left[\alpha(D-2)+\frac{2}{d}(2 \alpha+d \beta)\right] f(\varphi) d x \\
& \geq \frac{2}{d+1} \alpha \varepsilon \mathscr{L} F(\varphi) \geq \frac{2 \alpha \varepsilon \bar{\mu}}{d+1} F(\varphi),
\end{aligned}
$$

where we used (2-23) and (1-21) in the first inequality, $\min (1,2 / d) \geq 2 /(d+1)$ in the second, and $(2-24)$ in the last.

Using the inequalities above, we can replace the minimized quantity in (1-17) with a positive definite one, while extending the minimizing region from "the mountain ridge" to "the mountain flank". Let

$$
H_{\alpha, \beta}:=\left(1-\mathscr{L}_{\alpha, \beta} / \bar{\mu}\right) J .
$$

Then Lemma 2.2 implies that $H_{\alpha, \beta}>0$ and

$$
\mathscr{L}_{\alpha, \beta} H_{\alpha, \beta}=-(\mathscr{L}-\underline{\mu})(\mathscr{L}-\bar{\mu}) J / \bar{\mu}+\underline{\mu}(1-\mathscr{L} / \bar{\mu}) J \geq \frac{2 \alpha \varepsilon}{d+1} F+\underline{\mu} H_{\alpha, \beta} \geq 0 .
$$

We can rewrite the minimization problem (1-17) by using $H$ :

Lemma 2.3 (Minimization of $H$ ). Assume that $f$ satisfies (1-36) and $(\alpha, \beta)$ satisfies (1-16). Then $m_{\alpha, \beta}$ in (1-17) equals

$$
m_{\alpha, \beta}=\inf \left\{H_{\alpha, \beta}(\varphi) \mid \varphi \in H^{1}\left(\mathbb{R}^{d}\right), \varphi \neq 0, K_{\alpha, \beta}(\varphi) \leq 0\right\} .
$$

Proof. Let $m^{\prime}$ denote the right side of (2-28). Then $m \geq m^{\prime}$ is trivial because $J=H$ if $K=0$, so it suffices to show $m \leq m^{\prime}$. Take $\varphi \in H^{1}$ such that $K(\varphi)<0$.

If $(d, \alpha) \neq(2,0)$, then from Lemma 2.1 together with (2-3), we deduce that

$$
(d, \alpha) \neq(2,0), K(\varphi)<0 \Longrightarrow \exists \lambda<0, K\left(\varphi^{\lambda}\right)=0, H\left(\varphi^{\lambda}\right) \leq H(\varphi),
$$

where the latter inequality follows from (2-27) since $H\left(\varphi^{\lambda}\right)$ is nondecreasing in $\lambda$. Hence $m \leq m^{\prime}$.

If $(d, \alpha)=(2,0)$, then we use another scaling $v u$ with $v \in(0,1)$. We have $K^{Q}(v \varphi)=v^{2} K^{Q}(\varphi)$ and $\left|K^{N}(v \varphi)\right|=o\left(v^{4}\right)$ by (2-7) or (2-14). Hence $K(v \varphi)>0$ for small $v>0$, and so we deduce

$$
(d, \alpha)=(2,0), K(\varphi)<0 \Longrightarrow \exists v \in(0,1), K(v \varphi)=0, H(v \varphi) \leq H(\varphi),
$$

where the inequality follows from $H(\varphi)=\|\nabla \varphi\|_{L_{x}^{2}}^{2} / 2$ in this case. Hence $m \leq m^{\prime}$.

The ground state as common minimizer. Now we can prove the parameter independence of $m_{\alpha, \beta}$ via its characterization by the ground states. First we consider the $H^{1}$ subcritical case.

Lemma 2.4 (Ground state in the subcritical case). Assume that $f$ satisfies (1-36) and (1-26), and that $(\alpha, \beta)$ satisfies (1-16). Then $m_{\alpha, \beta}$ in (1-17) is positive and independent of $(\alpha, \beta)$. Moreover $m_{\alpha, \beta}=J(Q)$ for some $Q \in H^{1}\left(\mathbb{R}^{d}\right)$ solving the static $N L K G(1-39)$ with the minimal $J(Q)$ among the solutions in $H^{1}\left(\mathbb{R}^{d}\right)$. 
Proof. Let $\varphi_{n} \in H^{1}$ be a minimizing sequence for (2-28), namely $K\left(\varphi_{n}\right) \leq 0, \varphi_{n} \neq 0$ and $H\left(\varphi_{n}\right) \searrow m$.

First we consider the case $(d, \alpha) \neq(2,0)$. Let $\varphi_{n}^{*}$ be the Schwartz symmetrization of $\varphi_{n}$, i.e. the radial decreasing rearrangement. Since the symmetrization preserves the nonlinear parts and does not increase the $\dot{H}^{1}$ part, we have $\varphi_{n}^{*} \neq 0, K\left(\varphi_{n}^{*}\right) \leq K\left(\varphi_{n}\right) \leq 0$ and $H\left(\varphi_{n}\right) \geq H\left(\varphi_{n}^{*}\right) \rightarrow m$. Then using (2-29), we may replace it by symmetric $\psi_{n} \in H^{1}$ such that

$$
\psi_{n} \neq 0, \quad K\left(\psi_{n}\right)=0, \quad J\left(\psi_{n}\right)=H\left(\psi_{n}\right) \rightarrow m .
$$

If $\alpha>0$, then (2-17) implies

$$
(\bar{\mu}+\alpha \varepsilon) J\left(\psi_{n}\right) \geq \alpha \varepsilon\left\|\psi_{n}\right\|_{H^{1}}^{2} / 2
$$

hence $\psi_{n}$ is bounded in $H^{1}$.

If $\alpha=0$ (and $d>2$ ), then $H\left(\psi_{n}\right)=\left\|\nabla \psi_{n}\right\|_{L_{x}^{2}}^{2} / d$ is bounded, and if $\left\|\psi_{n}\right\|_{L^{2}} \rightarrow \infty$, then by (2-7)

$$
d \beta\left\|\psi_{n}\right\|_{L^{2}}^{2} \leq 2 K^{Q}\left(\psi_{n}\right)=-2 K^{N}\left(\psi_{n}\right) \leq o\left(\left\|\psi_{n}\right\|_{L^{2}}^{d-2_{\star}(d-2) / 2}\right),
$$

but since $d-2 \star(d-2) / 2<2$, this is a contradiction. Hence $\psi_{n}$ is bounded in $H^{1}$.

Since $\psi_{n}$ is bounded in $H^{1}$, after replacing with some appropriate subsequence, it converges to some $\psi$ weakly in $H^{1}$. By the radial symmetry, it also converges strongly in $L^{p}$ for all $2<p<2^{\star}$. Then in the subcritical case (1-26), the nonlinear parts converge, and so $K(\psi) \leq 0$ and $H(\psi) \leq m$.

If $\psi=0$, then $K\left(\psi_{n}\right)=0$ implies that $K^{Q}\left(\psi_{n}\right)=-K^{N}\left(\psi_{n}\right) \rightarrow 0$, and by Lemma 2.1 we have $K\left(\psi_{n}\right)>0$ for large $n$, a contradiction. Hence $\psi \neq 0$.

By (2-29), we may replace $\psi$ by its rescaling, so that $K(\psi)=0, J(\psi)=H(\psi) \leq m$ and $\psi \neq 0$. Then $\psi$ is a minimizer and $m=H(\psi)>0$.

Since $\psi$ is a minimizer for (1-17), there is a Lagrange multiplier $\eta \in \mathbb{R}$ such that

$$
J^{\prime}(\psi)=\eta K^{\prime}(\psi)
$$

Then denoting $\mathscr{L} \psi=\left.\partial_{\lambda} \psi_{\alpha, \beta}^{\lambda}\right|_{\lambda=0}$, we get

$$
0=K(\psi)=\mathscr{L} J(\psi)=\left\langle J^{\prime}(\psi) \mid \mathscr{L} \psi\right\rangle=\eta\left\langle K^{\prime}(\psi) \mid \mathscr{L} \psi\right\rangle=\eta \mathscr{L}^{2} J(\psi) .
$$

By $(2-18)$ and $\mathscr{L} J(\psi)=0$, we have

$$
\mathscr{L}^{2} J(\psi) \leq-\bar{\mu} \underline{\mu} J(\psi)-\frac{2 \alpha \varepsilon \bar{\mu}}{d+1} F(\psi)<0,
$$

since $\underline{\mu}>0$ or $\alpha>0$.

Therefore $\eta=0$ and $\psi$ is a solution to (1-39). The minimality of $J(\psi)$ among the solutions is clear from (1-17), since every solution $Q$ in $H^{1}$ of (1-39) satisfies $K(Q)=\left\langle J^{\prime}(Q) \mid \mathscr{L} Q\right\rangle=0$. This implies that $m_{\alpha, \beta}$ is independent of $(\alpha, \beta)$.

In the exceptional case $(d, \alpha)=(2,0)$, the above argument needs considerable modifications, due to the scaling invariance

$$
H(\varphi)=\|\nabla \varphi\|_{L^{2}}^{2} / 2=H\left(\varphi^{\lambda}\right), \quad K\left(\varphi^{\lambda}\right)=e^{d \beta \lambda} K(\varphi) .
$$


First, we should use (2-30) instead of (2-29) to get $\psi_{n}$ satisfying (2-31). Next, the invariance breaks the $H^{1}$ boundedness of $\psi_{n}$. But we are free to replace each $\psi_{n}$ by its rescaling so that $\left\|\psi_{n}\right\|_{L^{2}}=1$, without losing its properties (2-31). Then

$$
1=\left\|\psi_{n}\right\|_{L^{2}}^{2}=2 F\left(\psi_{n}\right) \rightarrow 2 F(\psi)
$$

which clearly implies that the limit $\psi$ is nonzero. By (2-30), we may replace $\psi$ by its constant multiple, so that $K(\psi)=0, J(\psi)=H(\psi) \leq m$ and $\psi \neq 0$. Then $\psi$ is a minimizer and $m=H(\psi)>0$.

Finally, the invariance gives us $\mathscr{L}^{2} J(\psi)=0$ and the Lagrange multiplier $\eta$ may be nonzero. In this case (2-34) is written

$$
-\Delta \psi=(\eta d \beta-1)\left[\psi-f^{\prime}(\psi)\right]
$$

Since $\langle-\Delta \psi \mid \psi\rangle_{L_{x}^{2}}>0$ and

$$
\left\langle\psi-f^{\prime}(\psi) \mid \psi\right\rangle_{L_{x}^{2}}=K_{0,2 / d}(\psi)-\int(D-2) f(\psi) d x<0,
$$

we have $(\eta d \beta-1)<0$. Hence there exists $\lambda>0$ such that $\psi^{\lambda}$ solves the static NLKG (1-39), and it is also a minimizer.

$\boldsymbol{H}^{1}$ critical case; massless threshold. In the $H^{1}$ critical case (1-28), we still have the $(\alpha, \beta)$ independence, but $m_{\alpha, \beta}$ is equal to the massless energy of the massless ground state. This is a consequence of the invariance of the massless energy with respect to the $\dot{H}^{1}$ scaling.

Lemma 2.5 (Ground state in $H^{1}$ critical case). Assume that $f$ satisfies (1-28), and that $(\alpha, \beta)$ satisfies (1-16). Then $m_{\alpha, \beta}$ in (1-17) is positive and independent of $(\alpha, \beta)$. Moreover $m_{\alpha, \beta}=J^{(0)}(Q)$ for some $Q \in \dot{H}^{1}\left(\mathbb{R}^{d}\right)$ solving the static massless $N L K G(1-41)$, with the minimal $J^{(0)}(Q)$ among the solutions in $\dot{H}^{1}\left(\mathbb{R}^{d}\right)$.

Proof. Let $H^{w}$ and $K^{w}$ be the massless versions of $H$ and $K$, respectively. Then

$$
m=m^{w}:=\inf \left\{H^{w}(\varphi) \mid \varphi \in H^{1}, K^{w}(\varphi)<0\right\} .
$$

Indeed, comparing this expression with (2-28), we easily get $m \geq m^{w}$ from $H^{w} \leq H$ and $K^{w}<K$ if $2 \alpha+d \beta>0$. If $2 \alpha+d \beta=0$, then we may replace $K \leq 0$ in (2-28) by $K<0$, because for any nonzero $\varphi \in H^{1}$ satisfying $K(\varphi) \leq 0$, we have by (2-18)

$$
\mathscr{L} K(\varphi) \leq \bar{\mu} K(\varphi)-\frac{2 \alpha \varepsilon \bar{\mu}}{d+1} F(\varphi)<0,
$$

which implies that $K\left(\varphi^{\lambda}\right)<0$ for all $\lambda>0$, and so the set $K<0$ is dense in the minimization set of (2-28). Hence $m \geq m^{w}$ in this case too.

To prove $m \leq m^{w}$, let

$$
\varphi^{v}=\varphi_{d / 2-1,-1}^{v}
$$

denote the $\dot{H}^{1}$ invariant scaling. Then $K\left(\varphi^{v}\right) \rightarrow K^{w}(\varphi)$ and $H\left(\varphi^{v}\right) \rightarrow H^{w}(\varphi)$ as $v \rightarrow \infty$. Hence if $K^{w}(\varphi)<0$ then $K\left(\varphi^{v}\right)<0$ for large $v$, and so $m \leq m^{w}$. 
Due to the $\dot{H}^{1}$ scale invariance, $K_{\alpha, \beta}^{w}$ for all $(\alpha, \beta)$ are constant multiples of the same functional, and $H^{w}$ is independent of $(\alpha, \beta)$, so is the minimization for $m^{w}$. In fact we have

$$
m^{w}=\inf \left\{\|\nabla \varphi\|_{L^{2}}^{2} / d \mid \varphi \in H^{1},\|\nabla \varphi\|_{L^{2}}^{2}<\|\varphi\|_{L^{2^{\star}}}^{2^{\star}}\right\} .
$$

By homogeneity and the scaling $\varphi \mapsto v \varphi$, this is equal to

$$
\inf _{0 \neq \varphi \in H^{1}} \frac{1}{d}\|\nabla \varphi\|_{L^{2}}^{2}\left[\frac{\|\nabla \varphi\|_{L^{2}}^{2}}{\|\varphi\|_{L^{2^{\star}}}^{2^{\star}}}\right]^{\frac{d-2}{2}}=\inf _{0 \neq \varphi \in H^{1}} \frac{1}{d}\left[\frac{\|\nabla \varphi\|_{L^{2}}}{\|\varphi\|_{L^{2^{\star}}}}\right]^{d}=\frac{\left(C_{S}^{\star}\right)^{-d}}{d},
$$

where $C_{S}^{\star}$ denotes the best constant for the Sobolev inequality

$$
\|\varphi\|_{L^{2^{\star}}} \leq C_{S}^{\star}\|\nabla \varphi\|_{L^{2}}
$$

which is well known to be attained by the explicit $Q \in \dot{H}^{1}$ given by

$$
Q(x)=\left[1+\frac{|x|^{2}}{d(d-2)}\right]^{-\frac{d-2}{2}}
$$

which solves (1-41).

Exponential case; mass-modified threshold. In the 2D exponential case (1-29), the conclusion is somewhat intermediate between the above two cases. If $C_{\mathrm{TM}}^{\star}(F) \geq 1$ then $m_{\alpha, \beta}$ is achieved by a ground state, but if $C_{\mathrm{TM}}^{\star}(F)<1$ then we can still see $m_{\alpha, \beta}$ as the energy of a ground state to an equation (1-43) where the mass is changed to $c=\min \left(1, C_{\mathrm{TM}}^{\star}(F)\right) \in(0,1)$.

Lemma 2.6 (Ground state in the exponential case). Assume that $f$ satisfies (1-36) and (1-29), and that $(\alpha, \beta)$ satisfies (1-16). Then $m_{\alpha, \beta}$ in (1-17) is independent of $(\alpha, \beta)$ and $0<m_{\alpha, \beta} \leq 2 \pi / \kappa_{0}$, where the second inequality is strict if and only if $C_{\mathrm{TM}}^{\star}(F)>1$. Moreover $m_{\alpha, \beta}=J^{(c)}(Q)$ with $c=\min \left(1, C_{\mathrm{TM}}^{\star}(F)\right)$ for some $Q \in H^{1}\left(\mathbb{R}^{2}\right)$ solving the modified static $N L K G(1-43)$ with the minimal $J^{(c)}(Q)$ among the solutions in $H^{1}\left(\mathbb{R}^{2}\right)$.

For the proof, we prepare some notations and lemmas. For any functional $G$ on $H^{1}\left(\mathbb{R}^{2}\right)$ and any $A>0$, we introduce the Trudinger-Moser ratio

$$
C_{\mathrm{TM}}^{A}(G):=\sup \left\{2 G(\varphi)\|\varphi\|_{L^{2}}^{-2} \mid 0 \neq \varphi \in H^{1}\left(\mathbb{R}^{2}\right),\|\nabla \varphi\|_{L^{2}} \leq A\right\},
$$

the Trudinger-Moser threshold on the $\dot{H}^{1}$ norm:

$$
\mathfrak{M}(G):=\sup \left\{A>0 \mid C_{\mathrm{TM}}^{A}(G)<\infty\right\},
$$

and the ratio on the threshold:

$$
C_{\mathrm{TM}}^{\star}(G):=C_{\mathrm{TM}}^{\mathfrak{M}(G)}(G) .
$$

The growth condition (1-29) together with (1-21) implies

$$
\mathfrak{M}\left(\mathscr{L}_{\alpha, \beta} F\right)=\mathfrak{M}(F)=\sqrt{4 \pi / \kappa_{0}}
$$

for any $(\alpha, \beta)$ satisfying (1-16), by the Trudinger-Moser inequality (2-13). Hence the definition of $C_{\mathrm{TM}}^{\star}$ just given is consistent with (1-30). 
For any functional $G$ of the form $G(\varphi)=\int g(\varphi) d x$, and for any sequence $\left(\varphi_{n}\right)_{n \in \mathbb{N}} \in H^{1}\left(\mathbb{R}^{2}\right)^{\mathbb{N}}$, we define its concentration (at $x=0) \operatorname{conc} G\left(\left(\varphi_{n}\right)_{n \in \mathbb{N}}\right.$ ) by

$$
\operatorname{conc} G\left(\left(\varphi_{n}\right)_{n \in \mathbb{N}}\right):=\varlimsup_{\varepsilon \rightarrow+0} \varlimsup_{n \rightarrow \infty} \int_{|x|<\varepsilon} g\left(\varphi_{n}\right) d x \text {. }
$$

We will use the following compactness by dominated convergence.

Lemma 2.7. Let $g, h: \mathbb{R} \rightarrow \mathbb{R}$ be continuous functions satisfying

$$
\lim _{u \rightarrow \pm \infty} \frac{|g(u)|}{h(u)}=0, \quad \lim _{u \rightarrow 0} \frac{|g(u)|}{|u|^{2}}=0 .
$$

Let $\varphi_{n}$ be a sequence of radial functions, weakly convergent to $\varphi$ in $H^{1}\left(\mathbb{R}^{2}\right)$ such that $\left\{h\left(\varphi_{n}\right)\right\}_{n}$ is bounded in $L^{1}\left(\mathbb{R}^{2}\right)$. Then $g\left(\varphi_{n}\right) \rightarrow g(\varphi)$ strongly in $L^{1}\left(\mathbb{R}^{2}\right)$.

Proof. By assumption (2-52), for any $\varepsilon>0$ there exist $\delta>0$ such that

$$
|u|>1 /(2 \delta) \text { or }|u|<2 \delta \Longrightarrow|g(u)|<\varepsilon\left(h(u)+|u|^{2}\right)
$$

Then we have

$$
\int_{\left|\varphi_{n}\right|>1 /(2 \delta) \text { or }\left|\varphi_{n}\right|<2 \delta}\left|g\left(\varphi_{n}\right)\right| d x \lesssim \varepsilon \int h\left(\varphi_{n}\right)+\left|\varphi_{n}\right|^{2} d x \lesssim \varepsilon .
$$

The radial Sobolev inequality $\left\|r^{1 / 2} \varphi_{n}\right\|_{L^{\infty}} \lesssim\left\|\varphi_{n}\right\|_{L^{2}}^{1 / 2}\left\|\nabla \varphi_{n}\right\|_{L^{2}}^{1 / 2}$ implies that $\varphi_{n}(x)$ are uniformly small for large $x$. Then the weak convergence together with

$$
\varphi_{n}\left(R_{1}\right)-\varphi_{n}\left(R_{2}\right)=\int_{R_{1}}^{R_{2}} \partial_{r} \varphi_{n}(r) d r
$$

implies that $\varphi_{n}(x) \rightarrow \varphi(x)$ for $x \neq 0$. Then Fatou's lemma implies

$$
\int_{|\varphi|>1 /(2 \delta) \text { or }|\varphi|<2 \delta}|g(\varphi)| d x \lesssim \varepsilon
$$

and the dominated convergence theorem implies

$$
\left\|g^{(\delta)}\left(\varphi_{n}\right)-g^{(\delta)}(\varphi)\right\|_{L^{1}} \rightarrow 0 \quad(n \rightarrow \infty),
$$

where $g^{(\delta)}$ is defined by $g^{(\delta)}(u)=\left(1-\chi_{\delta}(u)\right) \chi_{1 / \delta}(u) g(u)$ using the cut-off defined in (1-23). Combining (2-54), (2-56) and (2-57), we deduce the desired convergence.

Proof of Lemma 2.6. We start with the exceptional case $(d, \alpha)=(2,0)$. First, let $A>0$ and assume $C_{\mathrm{TM}}^{A}(F)>1$. Then there exists $0 \neq \varphi \in H^{1}$ such that $\|\nabla \varphi\|_{L^{2}} \leq A$ and $F(\varphi)>\|\varphi\|_{L^{2}}^{2} / 2$. For small $\varepsilon>0$ we have $K_{0,1}((1-\varepsilon) \varphi)<0$, and hence $m_{0,1} \leq\left\|\nabla(1-\varepsilon) \varphi_{n}\right\|_{L^{2}}^{2} / 2<A^{2} / 2$. Hence $m_{0,1} \leq \mathfrak{M}(F)^{2} / 2$.

Consider the case $C_{\mathrm{TM}}^{\star}(F)>1$. Then by choosing $A=\mathfrak{M}(F)$ in the above argument, we get $m_{0,1}<\mathfrak{M}(F)^{2} / 2$. Now we take a minimizing sequence for $m_{0,1}$. By the Schwartz symmetrization and rescalings as in the proof of Lemma 2.4 for $(d, \alpha)=(2,0)$, we get a sequence of radial functions $\psi_{n} \in H^{1}$ such that

$$
\left\|\psi_{n}\right\|_{L^{2}}=1, \quad H_{0,1}\left(\psi_{n}\right) \rightarrow m_{0,1}, \quad K_{0,1}\left(\psi_{n}\right)=1-2 F\left(\psi_{n}\right)=0,
$$


and $\psi_{n} \rightarrow \psi$ in $H^{1}$. Because of $m_{0,1}<\mathfrak{M}(F)^{2} / 2$, we can choose some $\kappa \in\left(\kappa_{0}, 2 \pi / m_{0,1}\right)$, so that $e^{\kappa\left|\psi_{n}\right|^{2}}-1$ is bounded in $L^{1}$ by the Trudinger-Moser inequality (2-13). Then we can use Lemma 2.7 with $\varphi_{n}:=\psi_{n}, g:=f$ and $h(u):=e^{\kappa|u|^{2}}-1$, which implies $F\left(\psi_{n}\right) \rightarrow F(\psi)$. Hence $\psi$ attains $m_{0,1}$. After appropriate rescalings, we obtain a ground state $Q$, as in the proof of Lemma 2.4.

Next consider the case $C_{\mathrm{TM}}^{\star}(F) \leq 1$. Then for any $\psi \in H^{1}$ satisfying $\|\nabla \psi\|_{L^{2}} \leq \mathfrak{M}(F)$ we have $K_{0,1}(\psi) \geq 0$. Hence

$$
m_{0,1}=\inf \left\{\|\nabla \varphi\|_{L^{2}}^{2} / 2 \mid K_{0,1}(\varphi)<0\right\} \geq \mathfrak{M}(F)^{2} / 2,
$$

and so $m_{0,1}=\mathfrak{M}(F)^{2} / 2$. Now we show that there exists $\varphi \in H^{1}$ satisfying

$$
\|\nabla \varphi\|_{L^{2}}=\mathfrak{M}(F), \quad F(\varphi)=C_{\mathrm{TM}}^{\star}(F) / 2, \quad\|\varphi\|_{L^{2}}=1 .
$$

After rescaling this $\varphi$, we obtain a ground state $Q$. However, due to the criticality, we have to approximate the problem by a subcritical one, namely we first prove the existence of $\varphi_{n} \in H^{1}$ satisfying

$$
\left\|\nabla \varphi_{n}\right\|_{L^{2}} \leq \mathfrak{M}(F)-\frac{1}{n}, \quad F\left(\varphi_{n}\right)=c_{n} / 2, \quad\left\|\varphi_{n}\right\|_{L^{2}}=1
$$

where $c_{n}:=C_{\mathrm{TM}}^{\mathfrak{M}(F)-\frac{1}{n}}(F)$; then $0<c_{n} \nearrow C_{\mathrm{TM}}^{\star}(F) \leq 1$. Fix $n \gg 1$ and let $\varphi^{k} \in H^{1}\left(\mathbb{R}^{2}\right)$ be a maximizing sequence for $c_{n}$ (see (2-47)):

$$
\left\|\nabla \varphi^{k}\right\|_{L^{2}} \leq \mathfrak{M}(F)-\frac{1}{n}, \quad F\left(\varphi^{k}\right) \nearrow c_{n} / 2, \quad\left\|\varphi^{k}\right\|_{L^{2}}=1
$$

where the $L^{2}$ norm is normalized by the rescaling $\varphi_{0,1}^{\lambda}$. The Schwartz symmetrization enables us to assume that $\varphi^{k}$ are radial functions, and convergent to some $\varphi_{n}$ weakly in $H^{1}$, by extracting a subsequence. Moreover, we have $F\left(\varphi^{k}\right) \rightarrow F\left(\varphi_{n}\right)=c_{n} / 2$, by Lemma 2.7 with $g:=f$ and $h=e^{\kappa|u|^{2}}-1$ for some $\kappa \in\left(\kappa_{0}, 4 \pi /(\mathfrak{M}(F)-1 / n)^{2}\right)$.

Thus $\varphi_{n}$ is a maximizer, which implies that $\left\|\varphi_{n}\right\|_{L^{2}}=1$ and

$$
-\eta \Delta \varphi_{n}=f^{\prime}\left(\varphi_{n}\right)-c_{n} \varphi_{n}
$$

for a Lagrange multiplier $\eta(n) \in \mathbb{R}$. Multiplying it with $\varphi_{n}$, we obtain

$$
\eta\left\|\nabla \varphi_{n}\right\|_{L^{2}}^{2}=\int D f\left(\varphi_{n}\right) d x-c_{n}\left\|\varphi_{n}\right\|_{L^{2}}^{2}=\int(D-2) f\left(\varphi_{n}\right) d x>0,
$$

since $(D-2) f>0$. Hence $\eta>0$, and so $Q_{n}(x):=\varphi_{n}\left(\eta^{1 / 2} x\right) \in H^{1}$ satisfies

$$
\left\|\nabla Q_{n}\right\|_{L^{2}} \leq \mathfrak{M}(F)-\frac{1}{n}, \quad-\Delta Q_{n}+c_{n} Q_{n}=f^{\prime}\left(Q_{n}\right)
$$

Now consider the limit $n \rightarrow \infty$. The equation for $Q_{n}$ implies that $0=K_{0,1}^{\left(c_{n}\right)}\left(Q_{n}\right)=K_{1,-1}^{\left(c_{n}\right)}\left(Q_{n}\right)$, so

$$
c_{n}\left\|Q_{n}\right\|_{L^{2}}^{2}=2 F\left(Q_{n}\right), \quad\left\|\nabla Q_{n}\right\|_{L^{2}}^{2}=2 \int(D-2) f\left(Q_{n}\right) d x \geq 4 F\left(Q_{n}\right),
$$

where the last inequality follows from $(D-4) f \geq 0$. Since $\left\|\nabla Q_{n}\right\|_{L^{2}}$ is bounded and $c_{n}$ is positive non-decreasing, we deduce that $\left\|Q_{n}\right\|_{L^{2}}$ and $\int D f\left(Q_{n}\right) d x$ are bounded as $n \rightarrow \infty$. Hence we may 
extract a subsequence so that $Q_{n}$ converges to some $Q$ weakly in $H^{1}$, and then apply Lemma 2.7 with $\varphi_{n}:=Q_{n}, g=f^{\prime}$ and $h:=D f$. Then $f^{\prime}\left(Q_{n}\right) \rightarrow f^{\prime}(Q)$ strongly in $L^{1}$, and so $Q$ solves

$$
-\Delta Q+c Q=f^{\prime}(Q), \quad c:=C_{\mathrm{TM}}^{\star}(F) .
$$

This implies that

$$
K_{0,1}^{(c)}(Q)=\left\langle J^{(c)^{\prime}}(Q) \mid \mathscr{L}_{0,1} Q\right\rangle=0,
$$

namely $2 F(Q)=c\|Q\|_{L^{2}}^{2}$. Hence $Q$ is a maximizer for $C_{\mathrm{TM}}^{\mathfrak{M}(F)}(F)$ with a non-zero Lagrange multiplier, which implies that $\|\nabla Q\|_{L^{2}}=\mathfrak{M}(F)$. Thus $J^{(c)}(Q)=\mathfrak{M}(F)^{2} / 2$ is unique for any solution $Q$ of (2-67).

Next we consider $m_{\alpha, \beta}$ with $\alpha>0$. If $m_{0,1}<\mathfrak{M}(F)^{2} / 2$, then there exists a ground state $Q$, which satisfies $K_{\alpha, \beta}(Q)=0$ for all $(\alpha, \beta)$. Hence $m_{\alpha, \beta} \leq J(Q)=m_{0,1}$.

Otherwise, $m_{0,1}=\mathfrak{M}(F)^{2} / 2=\mathfrak{M}(\mathscr{L} F)^{2} / 2$. For any $A>\mathfrak{M}(\mathscr{L} F)$, there exists a sequence $\varphi_{n} \in H^{1}$ satisfying

$$
\left\|\nabla \varphi_{n}\right\|_{L^{2}} \leq A, \quad\left\|\varphi_{n}\right\|_{L^{2}} \rightarrow 0, \quad \mathscr{L} F\left(\varphi_{n}\right) \rightarrow \infty .
$$

Since $K(\varphi)=\alpha\|\nabla \varphi\|_{L^{2}}^{2}+(\alpha+\beta)\|\varphi\|_{L^{2}}^{2}-\mathscr{L} F(\varphi)$ and $\alpha>0$, we can replace each $\varphi_{n}$ with $\varphi_{n}\left(x / v_{n}\right)$ with some $v_{n} \rightarrow+0$, so that we have after the rescaling

$$
\left\|\nabla \varphi_{n}\right\|_{L^{2}} \leq A, \quad K\left(\varphi_{n}\right)=0, \quad\left\|\varphi_{n}\right\|_{L^{2}} \rightarrow 0 .
$$

Hence

$$
m_{\alpha, \beta} \leq \lim _{n \rightarrow \infty} J\left(\varphi_{n}\right) \leq A^{2} / 2,
$$

and so $m_{\alpha, \beta} \leq \mathfrak{M}(\mathscr{L} F)^{2} / 2=m_{0,1}$. Thus in both cases we have $m_{\alpha, \beta} \leq m_{0,1} \leq \mathfrak{M}(F)^{2} / 2$.

Now suppose that $m_{\alpha, \beta}<m_{0,1} \leq \mathfrak{M}(F)^{2} / 2$. As in the proof of Lemma 2.4 for $(d, \alpha) \neq(2,0)$, we may find a sequence of radial $\varphi_{n} \in H^{1}$ such that

$$
K\left(\varphi_{n}\right)=0, \quad H\left(\varphi_{n}\right) \searrow m .
$$

Therefore there exists $\varphi$ such that $\varphi_{n} \rightarrow \varphi$ weakly in $H^{1}$, and pointwise for $x \neq 0$.

Let $\psi_{n}=\varphi_{n}-\varphi$. Then $\psi_{n} \rightarrow 0$ weakly in $H^{1}$, and so

$$
\lim _{n \rightarrow \infty} K^{Q}\left(\varphi_{n}\right)=\lim _{n \rightarrow \infty} K^{Q}\left(\psi_{n}\right)+K^{Q}(\varphi)=\lim _{n \rightarrow \infty} \mathscr{L} F\left(\varphi_{n}\right)=\operatorname{conc} \mathscr{L} F\left(\left(\varphi_{n}\right)_{n}\right)+\mathscr{L} F(\varphi),
$$

where the second identity is because $K\left(\varphi_{n}\right)=0$, and the last one follows from $\varphi_{n}(x) \rightarrow \varphi(x)$ for $x \neq 0$ and the radial Sobolev inequality $\left\|r^{1 / 2} \varphi_{n}\right\|_{L^{\infty}} \lesssim\left\|\varphi_{n}\right\|_{H^{1}}$. Since $H(\varphi) \leq m$ by Fatou's lemma, we have $K(\varphi) \geq 0$, otherwise there would be some $\lambda<0$ such that $K\left(\varphi^{\lambda}\right)=0$ and $H\left(\varphi^{\lambda}\right)<H(\varphi) \leq m$, a contradiction. Thus $K^{Q}(\varphi) \geq \mathscr{L} F(\varphi)$, and so from (2-72), we deduce

$$
\lim _{n \rightarrow \infty} K^{Q}\left(\psi_{n}\right) \leq \operatorname{conc} \mathscr{L} F\left(\left(\varphi_{n}\right)_{n}\right) .
$$

Since $\mathscr{L} F\left(\varphi_{n}\right)$ is bounded by (2-72), Lemma 2.7 with $h_{n}:=(\alpha D+\beta d) f$ implies that conc $F\left(\left(\varphi_{n}\right)_{n}\right)=$ 0 . Hence by $(2-73)$ and $(\mathscr{L}-\bar{\mu}) F \geq 0$, we get

$$
\lim _{n \rightarrow \infty} K^{Q}\left(\psi_{n}\right) \leq \operatorname{conc}(\mathscr{L}-\bar{\mu}) F\left(\left(\varphi_{n}\right)_{n}\right) \leq \lim _{n \rightarrow \infty}(\mathscr{L}-\bar{\mu}) F\left(\varphi_{n}\right) .
$$


On the other hand we have

$$
m=\lim _{n \rightarrow \infty} H\left(\varphi_{n}\right)=\lim _{n \rightarrow \infty} H^{Q}\left(\psi_{n}\right)+H^{Q}(\varphi)+\lim _{n \rightarrow \infty}(\mathscr{L}-\bar{\mu}) F\left(\varphi_{n}\right) / \bar{\mu},
$$

where $H^{Q}(\psi):=(1-\mathscr{L} / \bar{\mu})\|\psi\|_{H^{1}}^{2} / 2$ denotes the quadratic part of $H$. Combining the above two, and discarding $H^{Q}(\varphi) \geq 0$, we obtain

$$
\lim _{n \rightarrow \infty}\left\|\psi_{n}\right\|_{H^{1}}^{2} / 2 \leq m<\mathfrak{M}(F)^{2} / 2=2 \pi / \kappa_{0}
$$

Hence applying Lemma 2.7 to $\varphi_{n}$ with $h(u):=e^{\kappa|u|^{2}}-1$ for some $\kappa \in\left(\kappa_{0}, 2 \pi / m\right)$, we get $\mathscr{L} F\left(\varphi_{n}\right) \rightarrow$ $\mathscr{L} F(\varphi)$, and so $\varphi$ is a minimizer for $m_{\alpha, \beta}$. Indeed, we have

$$
e^{\kappa\left|\varphi_{n}\right|^{2}}-1 \leq e^{C_{\kappa, \kappa^{\prime}}|\varphi|^{2}}-1+e^{\kappa^{\prime}\left|\psi_{n}\right|^{2}}-1
$$

for some $\kappa^{\prime} \in(\kappa, 2 \pi / m)$ and constant $C_{\kappa, \kappa^{\prime}}>0$. Hence $h\left(\varphi_{n}\right)$ is uniformly bounded in $L^{1}$. Recall that for a fixed $\varphi \in H^{1}, e^{C_{\kappa, \kappa^{\prime}}|\varphi|^{2}}-1 \in L^{1}$.

Then as in the proof of Lemma 2.4, we obtain a ground state $Q$ with $J(Q)=m_{\alpha, \beta}<m_{0,1}$, which is a contradiction since $K_{0,1}(Q)=0$. Hence $m_{\alpha, \beta}=m_{0,1}$ for all $(\alpha, \beta)$ in the range $(1-16)$.

Remark 2.8. In the above argument for $(\alpha, \beta)=(0,1)$ in the case $C_{\mathrm{TM}}^{\star}(F) \leq 1$, we used a priori bounds on the ground state to get the compactness. For general sequences, we can have concentrating loss of compactness on the kinetic threshold $\|\nabla \varphi\|_{L^{2}}=\mathfrak{M}(F)$ if and only if $f$ satisfies

$$
\varlimsup_{|u| \rightarrow \infty} e^{-\kappa_{0}|u|^{2}}|u|^{2} f(u) \in(0, \infty) .
$$

Lemma 2.6 implies that the concentration requires more energy than the (mass-modified) ground state. Similar phenomena have been observed in slightly different settings (either on a bounded domain or on the $H^{1}\left(\mathbb{R}^{2}\right)$ threshold, where $e^{\kappa_{0}|u|^{2}}$ appears as the critical growth instead of $e^{\kappa_{0}|u|^{2}} /|u|^{2}$, see [Carleson and Chang 1986; Flucher 1992; Ruf 2005]). More details about this issue, including the above concentration compactness, will be addressed in a forthcoming paper.

Parameter independence of the splitting. The $(\alpha, \beta)$-independence of $\mathscr{Y}_{\alpha, \beta}^{ \pm}$follows from that of $m_{\alpha, \beta}$ and contractivity of $\mathscr{K}_{\alpha, \beta}^{+}$.

Lemma 2.9 (Parameter independence of $\left.\mathscr{K}^{ \pm}\right)$. Assume that $f$ satisfies (1-36), and that $(\alpha, \beta)$ satisfies (1-16). Then $\mathcal{K}_{\alpha, \beta}^{ \pm}$in (1-18) are independent of $(\alpha, \beta)$.

Proof. Since $m_{\alpha, \beta}$ is independent of $(\alpha, \beta)$, we only need to see that the sign of $K$ is independent under the threshold $m$. Also, we may restrict to the first component. For any $\delta \geq 0$, we define $\mathcal{T}_{\alpha, \beta}^{ \pm \delta} \subset H^{1}$ by

$$
\begin{aligned}
& \mathcal{K}_{\alpha, \beta}^{+\delta}=\left\{\varphi \in H^{1} \mid J(\varphi)<m-\delta, K_{\alpha, \beta}(\varphi) \geq 0\right\}, \\
& \mathcal{K}_{\alpha, \beta}^{-\delta}=\left\{\varphi \in H^{1} \mid J(\varphi)<m-\delta, K_{\alpha, \beta}(\varphi)<0\right\} .
\end{aligned}
$$

Then $\left(u_{0}, u_{1}\right) \in \mathscr{K}_{\alpha, \beta}^{ \pm}$if and only if $u_{0} \in \mathscr{K}_{\alpha, \beta}^{ \pm \delta}$ with $\delta=\left\|u_{1}\right\|_{L^{2}}^{2} / 2$. In addition, the disjoint union $\mathscr{K}_{\alpha, \beta}^{+\delta} \cup \mathcal{K}_{\alpha, \beta}^{-\delta}$ is already independent of $\alpha$ and $\beta$. Hence it suffices to show the independence of $\mathscr{K}_{\alpha, \beta}^{+\delta}$.

First we consider the interior exponents satisfying $2 \alpha+d \beta>0$ and $2 \alpha+(d-2) \beta>0$. Then $\mathscr{K}_{\alpha, \beta}^{+\delta}$ is contracted to $\{0\}$ by the rescaling $\varphi \mapsto \varphi^{\lambda}$ with $0 \geq \lambda \rightarrow-\infty$. This is due to the following facts: 
(1) $K\left(\varphi^{\lambda}\right)>0$ is preserved as long as $J\left(\varphi^{\lambda}\right)<m$, by the definition of $m$.

(2) $J\left(\varphi^{\lambda}\right)$ does not increase as $\lambda$ decreases, as long as $\mathscr{L} J\left(\varphi^{\lambda}\right)=K\left(\varphi^{\lambda}\right)>0$.

(3) $\varphi^{\lambda} \rightarrow 0$ in $H^{1}$ as $\lambda \rightarrow-\infty$, since $2 \alpha+d \beta>0$ and $2 \alpha+(d-2) \beta>0$.

In particular, $J$ cannot be negative on $\mathscr{K}_{\alpha, \beta}^{+}$, and so $\mathcal{K}_{\alpha, \beta}^{+\delta}=\varnothing$ for $\delta \geq m$. For $0 \leq \delta<m$, both $\mathscr{K}_{\alpha, \beta}^{ \pm \delta}$ are open in $H^{1}$. It follows for $\mathscr{K}^{-\delta}$ from the definition, and for $\mathscr{K}^{+\delta}$ from the facts that $J(\varphi)<m$ and $K(\varphi)=0$ imply $\varphi=0$, and that a neighborhood of 0 is contained in $\mathcal{K}^{+\delta}$, which follows from (2-7), (2-8) or (2-14). Then the above argument of the scaling contraction shows that $\mathscr{K}_{\alpha, \beta}^{+\delta}$ is connected. Hence each $\mathcal{K}_{\alpha, \beta}^{+\delta}$ cannot be separated by $\mathcal{K}_{\alpha^{\prime}, \beta^{\prime}}^{+\delta}$ and $\mathscr{K}_{\alpha^{\prime}, \beta^{\prime}}^{-\delta}$ with any other $\left(\alpha^{\prime}, \beta^{\prime}\right)$ in the interior range. Since $\mathscr{K}_{\alpha, \beta}^{+\delta} \cap \mathscr{K}_{\alpha^{\prime}, \beta^{\prime}}^{+\delta}$ contains 0 , we conclude that $\mathscr{K}_{\alpha, \beta}^{+\delta}=\mathscr{K}_{\alpha^{\prime}, \beta^{\prime}}^{+\delta}$.

Finally for $(\alpha, \beta)$ on the boundary $2 \alpha+d \beta=0$ or $2 \alpha+(d-2) \beta=0$, take a sequence $\left(\alpha_{n}, \beta_{n}\right)$ in the interior converging to $(\alpha, \beta)$. Then $K_{\alpha_{n}, \beta_{n}} \rightarrow K_{\alpha, \beta}$, and so

$$
\mathscr{K}_{\alpha, \beta}^{ \pm \delta} \subset \bigcup_{n} \mathscr{K}_{\alpha_{n}, \beta_{n}}^{ \pm \delta}
$$

Since the right side is independent of the parameter, so is the left.

Variational estimates. We conclude this section with a few estimates on the energy-type functionals, which will be important in the proof of the blow-up and the scattering. We start with the easy observation that the free energy and the nonlinear energy are equivalent in the set $\mathscr{K}^{+}$.

Lemma 2.10 (Free energy equivalence in $\left.\mathscr{K}^{+}\right)$. Assume that $f$ satisfies (1-36). Then for any $\left(u_{0}, u_{1}\right) \in$ $H^{1}\left(\mathbb{R}^{d}\right) \times L^{2}\left(\mathbb{R}^{d}\right)$ we have

$$
K_{1,0}\left(u_{0}\right) \geq 0 \Longrightarrow\left\{\begin{array}{l}
J\left(u_{0}\right) \leq\left\|u_{0}\right\|_{H_{x}^{1}}^{2} / 2 \leq(1+d / 2) J\left(u_{0}\right) \\
E\left(u_{0}, u_{1}\right) \leq E^{Q}\left(u_{0}, u_{1}\right) \leq(1+d / 2) E\left(u_{0}, u_{1}\right)
\end{array}\right.
$$

Proof. Since $(D-2-c) f(u) \geq 0$ with $c:=4 / d>0$ by (1-21), we have for any $\left(u_{0}, u_{1}\right) \in H^{1} \times L^{2}$,

$$
\begin{aligned}
K_{1,0}\left(u_{0}\right) & =\left\|u_{0}\right\|_{H_{x}^{1}}^{2}-(2+c) F\left(u_{0}\right)-\int(D-2-c) f\left(u_{0}\right) d x \\
& \leq(2+c) J\left(u_{0}\right)-c\left\|u_{0}\right\|_{H_{x}^{1}}^{2} / 2=(2+c) E\left(u_{0}, u_{1}\right)-c E^{\left.Q_{\left(u_{0}\right.}, u_{1}\right)-\|\dot{u}\|_{L_{x}^{2}}^{2},}
\end{aligned}
$$

and hence we obtain the desired estimate.

In the $2 \mathrm{D}$ exponential case, we have a sharper bound on the derivatives, which implies that $\mathscr{K}^{+}$is in the subcritical regime for the Trudinger-Moser inequality.

Lemma 2.11 (Subcritical bound in $\mathscr{K}^{+}$in the 2D exponential case). Assume that $f$ satisfies (1-36) and (1-29). Then for any $\left(u_{0}, u_{1}\right) \in \mathscr{K}^{+}$we have

$$
\left\|\nabla u_{0}\right\|_{L^{2}}^{2}+\left\|u_{1}\right\|_{L^{2}}^{2}<2 m \leq \mathfrak{M}(F)^{2}=4 \pi / \kappa_{0} .
$$

Proof. Since $K_{0,1}\left(u_{0}\right) \geq 0$, we have

$$
\left\|\nabla u_{0}\right\|_{L^{2}}^{2}+\left\|u_{1}\right\|_{L^{2}}^{2} \leq\left\|\nabla u_{0}\right\|_{L^{2}}^{2}+\left\|u_{1}\right\|_{L^{2}}^{2}+K_{0,1}\left(u_{0}\right)=2 E\left(u_{0}, u_{1}\right)<2 m .
$$


The next estimate gives a lower bound on $|K|$ under the threshold $m$, which will be important both for the blow-up and for the scattering.

Lemma 2.12 (Uniform bounds on $K$ ). Assume that $f$ satisfies (1-21), and that $(\alpha, \beta)$ satisfies (1-16) and $(d, \alpha) \neq(2,0)$. Then there exists $\delta>0$ determined by $(\alpha, \beta), d$ and $\varepsilon$ in (1-21), such that for any $\varphi \in H^{1}$ with $J(\varphi)<m$ we have

$$
K_{\alpha, \beta}(\varphi) \geq \min \left(\bar{\mu}(m-J(\varphi)), \delta K_{\alpha, \beta}^{Q}(\varphi)\right) \quad \text { or } \quad K_{\alpha, \beta}(\varphi) \leq-\bar{\mu}(m-J(\varphi)) .
$$

Note that if $(d, \alpha)=(2,0)$ then the conclusion is false, since in that case

$$
K\left(\varphi_{\alpha, \beta}^{\lambda}\right)=e^{d \beta \lambda} K(\varphi) \rightarrow 0 \quad \text { as } \lambda \rightarrow-\infty,
$$

while $J\left(\varphi^{\lambda}\right)$ is away from $m$, since it is decreasing if $K(\varphi)>0$ and $J\left(\varphi^{\lambda}\right) \nearrow H(\varphi)<m$ if $K(\varphi)<0$. Proof. We may assume $\varphi \neq 0$. Let $j(\lambda)=J\left(\varphi^{\lambda}\right)$ and $n(\lambda)=F\left(\varphi^{\lambda}\right)$, where $\varphi_{\alpha, \beta}^{\lambda}=\varphi^{\lambda}$ is the rescaling (1-13). Then $j(0)=J(\varphi)$ and $j^{\prime}(0)=K(\varphi)$, and (2-18) implies

$$
j^{\prime \prime} \leq(\bar{\mu}+\underline{\mu}) j^{\prime}-\bar{\mu} \underline{\mu} j-\frac{2 \alpha \varepsilon}{d+1} n^{\prime} .
$$

First we consider the case $K(\varphi)<0$. By Lemma 2.1 together with (2-3), there exists $\lambda_{0}<0$ such that $j^{\prime}(\lambda)<0$ for $\lambda_{0}<\lambda \leq 0$ and $j^{\prime}\left(\lambda_{0}\right)=0$. For $\lambda_{0} \leq \lambda \leq 0$ we have from (2-16),

$$
(\bar{\mu}+\underline{\mu}) j^{\prime}-\bar{\mu} \underline{\mu} j \leq \bar{\mu} j^{\prime} .
$$

Inserting this in (2-86) and integrating it, we get

$$
\int_{\lambda_{0}}^{0} j^{\prime \prime}(\lambda) d \lambda \leq \bar{\mu} \int_{\lambda_{0}}^{0} j^{\prime}(\lambda) d \lambda
$$

and hence

$$
K(\varphi)=j^{\prime}(0) \leq \bar{\mu}\left(j(0)-j\left(\lambda_{0}\right)\right) .
$$

Since $K\left(\varphi^{\lambda_{0}}\right)=0$ and $\varphi^{\lambda_{0}} \neq 0$, we have $j\left(\lambda_{0}\right)=J\left(\varphi^{\lambda_{0}}\right) \geq m$. Thus we obtain

$$
K(\varphi) \leq-\bar{\mu}(m-J(\varphi)) .
$$

Next we consider the case $K(\varphi)>0$. If

$$
(2 \bar{\mu}+\underline{\mu}) K(\varphi) \geq \bar{\mu} \underline{\mu} J(\varphi)+\frac{2 \alpha \varepsilon}{d+1} \mathscr{L} F(\varphi),
$$

then applying (2-81) to the first term on the right-hand side, and $K=K^{Q}-\mathscr{L} F$ to the second one, we get

$$
\left[2 \bar{\mu}+\underline{\mu}+\frac{2 \alpha \varepsilon}{d+1}\right] K(\varphi) \geq \frac{\bar{\mu} \underline{\mu}}{2+d}\|\varphi\|_{H^{1}}^{2}+\frac{2 \alpha \varepsilon}{d+1} K^{Q}(\varphi),
$$

and so $K(\varphi) \geq \delta K^{Q}(\varphi)$ for some $\delta>0$, since $\underline{\mu}>0$ or $\alpha>0$. If (2-91) fails, then

$$
(2 \bar{\mu}+\underline{\mu}) j^{\prime}<\bar{\mu} \underline{\mu} j+\frac{2 \alpha \varepsilon}{d+1} n^{\prime},
$$


at $\lambda=0$, and so from $(2-86)$,

$$
j^{\prime \prime}<-\bar{\mu} j^{\prime} .
$$

Now let $\lambda$ increase. As long as (2-93) holds and $j^{\prime}>0$, we have $j^{\prime \prime}<0$ and so $j^{\prime}$ decreases and $j$ increases. Also by (2-18) and (2-16) we have

$$
n^{\prime \prime} \geq(\bar{\mu}+\underline{\mu}) n^{\prime}-\bar{\mu} \underline{\mu} n \geq \bar{\mu} n^{\prime} \geq \bar{\mu}^{2} n>0 .
$$

Hence (2-93) is preserved until $j^{\prime}$ reaches 0 . It does reach at finite $\lambda_{0}>0$, because the right-hand side of (2-86) is negative and decreasing as long as $j^{\prime}>0$. Now integrating (2-94) we obtain

$$
K(\varphi)=j^{\prime}(0) \geq \bar{\mu}\left(j\left(\lambda_{0}\right)-j(0)\right) \geq \bar{\mu}(m-J(\varphi)),
$$

where we used that $J\left(\varphi^{\lambda_{0}}\right) \geq m$ which follows from $K\left(\varphi^{\lambda_{0}}\right)=0$ and $\varphi^{\lambda_{0}} \neq 0$.

\section{Blow-up}

Here we prove the blow-up part of Theorem 1.1. The idea is essentially due to Payne and Sattinger [1975], but we give a full proof for convenience. We will use that $\mathscr{K}^{-}$is stable under the flow.

Assume for a contradiction that a solution $u$ exists for all $t>0$. (The proof for $t<0$ is the same.) Let

$$
y(t):=\|u(t, x)\|_{L_{x}^{2}\left(\mathbb{R}^{d}\right)}^{2}
$$

Multiplying the equation with $u$, and using (2-82), we get

$$
\ddot{y}=2\|\dot{u}\|_{L^{2}}^{2}-2 K_{1,0}(u) \geq(4+c)\|\dot{u}\|_{L^{2}}^{2}-2(2+c) E(u)+c\|u\|_{H^{1}}^{2},
$$

for some $c>0$. Sine $u(t) \in \mathscr{K}^{-}$, Lemma 2.12 implies that there is some positive $\delta \leq-K_{1,0}(u(t))$. Thus for all $t>0$ we have

$$
\ddot{y}(t) \geq 2 \delta>0,
$$

and so $y(t)=\|u(t)\|_{L^{2}}^{2} \rightarrow \infty$ as $t \rightarrow \infty$. Going back to (3-2), and using Schwarz, we deduce that for large $t$

therefore

$$
\ddot{y} \geq(4+c)\|\dot{u}\|_{L^{2}}^{2}>\frac{4+c}{4} \frac{\dot{y}^{2}}{y},
$$

$$
\left(y^{-c / 4}\right)_{t t}=-\frac{c}{4} y^{-c / 4-2}\left[y \ddot{y}-\frac{4+c}{4} \dot{y}^{2}\right]<0,
$$

which contradicts that $y \rightarrow \infty$.

\section{Global space-time norm}

In this section we introduce Strichartz-type estimates and a perturbation lemma for global space-time bounds of the solution.

The inhomogeneity of the Klein-Gordon equation makes the exponents a bit more complicated than the case of wave or Schrödinger equation. In the $H^{1}$ critical case, we get another complication in higher dimensions, due to the fact that we have to estimate the difference of solutions in some Sobolev (or Besov) spaces with positive regularity but the nonlinearity is not twice differentiable. ${ }^{2}$ This is not a

\footnotetext{
${ }^{2}$ The problem is not in the local regularity of the nonlinearity (at $u=0$ ), but rather in the global Hölder continuity for $f_{L}$.
} 
problem in the subcritical case, where we are allowed to lose small regularity, so that we can estimate the difference in some $L^{p}$ spaces and then interpolate. This technical issue was solved in the pure critical case in [Nakanishi 1999a] by using space-time norms with exponents away from the admissible region for the standard Strichartz estimate, which was later called "exotic Strichartz estimates" in the Schrödinger case [Tao and Visan 2005].

Here we have a further complication by the presence of lower powers, for which we need the exotic Strichartz for the Klein-Gordon equation. Note that it is not a big trouble in the Schrödinger case (see [Tao et al. 2007]), because the same Strichartz estimate is used both for higher and lower powers. In the Klein-Gordon case, in contrast, we have to use different Strichartz norms, with better regularity for higher powers and with better decay for lower powers. It is easy in the standard Strichartz estimate, where we can freely mix different norms by the duality argument, but this does not work for the exotic Strichartz estimate, which uses exponents away from the duality. Hence we are forced to use a common exponent for different powers, which makes our estimates much more involved. In particular, when we have both the $H^{1}$ critical and the $L^{2}$ critical powers, we need three steps to close our estimates.

Reduction to a first-order equation. To simplify the notation, we rewrite NLKG as a first-order equation. To any real-valued function $u(t, x)$, we associate a complex-valued ${ }^{3}$ function $\vec{u}(t, x)$ thus:

$$
\vec{u}=\langle\nabla\rangle u-i \dot{u}, \quad u=\langle\nabla\rangle^{-1} \operatorname{Re} \vec{u} .
$$

This relation $u \leftrightarrow \vec{u}$ will be assumed for any space-time function $u$ throughout this paper. The free and nonlinear Klein-Gordon equations are given by

$$
\begin{array}{ll}
(\square+1) u=0 & \text { (equivalently, } \left.\left(i \partial_{t}+\langle\nabla\rangle\right) \vec{u}=0\right), \\
(\square+1) u=f^{\prime}(u) & \text { (equivalently, } \left.\left(i \partial_{t}+\langle\nabla\rangle\right) \vec{u}=f^{\prime}\left(\langle\nabla\rangle^{-1} \operatorname{Re} \vec{u}\right)\right),
\end{array}
$$

and the free energy is given by $E^{Q}(u)=\|\vec{u}\|_{L_{x}^{2}}^{2} / 2$. We set

$$
\widetilde{E}(\varphi):=\|\varphi\|_{L_{x}^{2}}^{2} / 2-F\left(\langle\nabla\rangle^{-1} \operatorname{Re} \varphi\right), \quad \widetilde{K}_{\alpha, \beta}(\varphi):=K_{\alpha, \beta}^{Q}\left(\langle\nabla\rangle^{-1} \varphi\right)+K_{\alpha, \beta}^{N}\left(\langle\nabla\rangle^{-1} \operatorname{Re} \varphi\right)
$$

Remark that

$$
\widetilde{E}(\vec{u}(t))=E(u ; t), \quad \tilde{K}(\vec{u}(t)) \geq K(u(t)),
$$

where the inequality is an equality if and only if $\dot{u}(t)=0$. The invariant set $\mathscr{K}^{+}=\mathscr{K}_{\alpha, \beta}^{+}$for $\vec{u}$ is given by

$$
\begin{aligned}
\widetilde{\mathscr{K}}^{+} & :=\left\{\varphi \in L^{2}\left(\mathbb{R}^{d}\right) \mid \tilde{E}(\varphi)<m, K\left(\operatorname{Re}\langle\nabla\rangle^{-1} \varphi\right) \geq 0\right\} \\
& =\left\{\varphi \in L^{2}\left(\mathbb{R}^{d}\right) \mid \widetilde{E}(\varphi)<m, \widetilde{K}(\varphi) \geq 0\right\} .
\end{aligned}
$$

The second identity is proved as follows. Let $\varphi \in L^{2}\left(\mathbb{R}^{d}\right)$ satisfy $\widetilde{E}(\varphi)<m$ and $K\left(\operatorname{Re}\langle\nabla\rangle^{-1} \varphi\right)<0$. Let $\psi_{1}=\operatorname{Re}\langle\nabla\rangle^{-1} \varphi$ and $\psi_{2}=\operatorname{Im}\langle\nabla\rangle^{-1} \varphi$. Then Lemma 2.12 implies that

$$
K\left(\psi_{1}\right) \leq-\bar{\mu}\left(m-J\left(\psi_{1}\right)\right)<-\bar{\mu}\left\|\psi_{2}\right\|_{H_{x}^{1}}^{2} / 2 \leq-K^{Q}\left(\psi_{2}\right),
$$

\footnotetext{
${ }^{3}$ We do not need the complex structure; we use $i$ purely for notational convenience, and could use vector notation instead, especially if $u$ is originally complex-valued. We chose the complex form rather than the vector one to avoid adding a subscript, for this notation will be applied mostly to sequences.
} 
so $\widetilde{K}(\varphi)=K\left(\psi_{1}\right)+K^{Q}\left(\psi_{2}\right)<0$. Hence under the condition $\widetilde{E}(\varphi)<m$, the signs of $K\left(\psi_{1}\right)$ and $\widetilde{K}(\varphi)$ are the same, which proves (4-5).

Strichartz-type estimates and exponents. Here we recall the Strichartz estimate for the free KleinGordon equation, introducing some notation for the space-time norms and special exponents.

With any triplet $(b, c, \sigma) \in[0,1]^{2} \times \mathbb{R}$ and any $q \in(0, \infty]$, we associate the following Banach function spaces on $I \times \mathbb{R}^{d}$ for any interval $I$ :

$$
\begin{aligned}
& {[(b, c, \sigma)]_{q}(I):=L_{t}^{1 / b}\left(I ; B_{1 / c, q}^{\sigma}\left(\mathbb{R}^{d}\right)\right),} \\
& {[(b, c, \sigma)]_{0}(I):=L_{t}^{1 / b}\left(I ; L^{1 / c}\left(\mathbb{R}^{d}\right)\right),} \\
& {[(b, c, \sigma)]_{q}^{\bullet}(I):=L_{t}^{1 / b}\left(I ; \dot{B}_{1 / c, q}^{\sigma}\left(\mathbb{R}^{d}\right)\right),}
\end{aligned}
$$

where $B_{p, q}^{s}$ and $\dot{B}_{p, q}^{s}$ respectively denote the inhomogeneous and homogeneous Besov spaces, and the following characteristic numbers with a parameter $\theta \in[0,1]$ :

$$
\begin{aligned}
\operatorname{reg}^{\theta}(b, c, \sigma) & :=\sigma-(1-2 \theta / d) b-d\left(c-\frac{1}{2}\right), \\
\operatorname{str}^{\theta}(b, c, \sigma) & :=2 b+(d-1+\theta)\left(c-\frac{1}{2}\right), \\
\operatorname{dec}^{\theta}(b, c, \sigma) & :=b+(d-1+\theta)\left(c-\frac{1}{2}\right) .
\end{aligned}
$$

The cases $\theta=0,1$ correspond respectively to the wave and the Klein-Gordon equations. reg ${ }^{\theta}$ indicates the regularity of the space, while $\operatorname{str}^{\theta}$ and $\operatorname{dec}^{\theta}$ indicate the space-time decay, corresponding respectively to the Strichartz and the $L^{p}-L^{q}$ decay estimates. We denote the regularity change and the duality in $H^{s-1 / 2}$ (here $-\frac{1}{2}$ takes account of one regularity gain in the wave equation) respectively by

$$
(b, c, \sigma)^{s}:=(b, c, s), \quad(b, c, \sigma)^{*(s)}:=(1-b, 1-c,-\sigma+2 s-1) .
$$

Given a real number $s$, we say that $Z=\left(Z_{1}, Z_{2}, Z_{3}\right)$ is Strichartz $s$-admissible if for some $\theta \in[0,1]$ we have

$$
0 \leq Z_{1} \leq \frac{1}{2}, \quad 0 \leq Z_{2}<\frac{1}{2}, \quad \operatorname{reg}^{\theta}(Z) \leq s, \quad \operatorname{str}^{\theta}(Z) \leq 0 .
$$

We avoid the endpoint $Z_{2}=\frac{1}{2}$ to mix different $\theta$ 's. We now state the Strichartz estimates:

Lemma 4.1 (see [Brenner 1984; Ginibre and Velo 1985a; Machihara et al. 2002]). For any $s \in \mathbb{R}$, let $Z$ and $T$ be s-admissible. Then for any space-time function $u(t, x)$, any interval $I \subset \mathbb{R}$, and any $t_{0} \in I$, we have

$$
\|u\|_{[Z]_{2}(I)} \lesssim\left\|u\left(t_{0}\right)\right\|_{H^{s}}+\left\|\dot{u}\left(t_{0}\right)\right\|_{H^{s-1}}+\|\ddot{u}-\Delta u+u\|_{\left[T^{*(s)}\right]_{2}(I)},
$$

where the implicit constant does not depend on I or $t_{0}$.

The "exotic Strichartz estimate" is given for the Klein-Gordon equation by

Lemma 4.2. Let $Z, T \in \mathbb{R}^{3}$ satisfy for some $\theta \in[0,1]$

$$
\begin{aligned}
& \operatorname{reg}^{\theta}(Z) \leq \operatorname{reg}^{\theta}(T)+2, \quad \operatorname{str}^{\theta}(Z) \leq \operatorname{str}^{\theta}(T)-2, \quad 0<Z_{1}, T_{1}<1, \\
& \operatorname{dec}^{\theta}(Z)<0<\operatorname{dec}^{\theta}(T)-1, \quad 0<\frac{1}{2}-Z_{2}, T_{2}-\frac{1}{2}<\frac{1}{d-1+\theta} .
\end{aligned}
$$


Then, for any interval $I \subset \mathbb{R}, t_{0} \in I$, and $u(t, x)$ satisfying $u\left(t_{0}\right)=\dot{u}\left(t_{0}\right)=0$,

$$
\|u\|_{[Z]_{2}(I)} \lesssim\|\ddot{u}-\Delta u+u\|_{[T]_{2}(I)} .
$$

Proof. The wave case $\theta=0$ was essentially proved in [Nakanishi 1999a, Lemma 7.4], where the borderline case $\operatorname{str}^{0}(Z)=\operatorname{str}^{0}(T)-2$ was excluded for the real interpolation to improve the Besov exponent 2. Here we discard that improvement, restoring the borderline case, which is needed for the lower critical power $p_{1}=4 / d$.

The proof is rather immediate from the standard Strichartz estimate and the $L^{p}$ decay estimate. Indeed, if $\operatorname{str}^{\theta}(Z)=0=\operatorname{str}^{\theta}(T)-2$ and $\operatorname{reg}^{\theta}(Z)=\operatorname{reg}^{\theta}(T)+2$, then the above estimate is nothing but Strichartz. If moreover $Z_{2}+T_{2}=1$, then the estimate directly follows from the $L^{p}$ decay and the Hardy-Littlewood-Sobolev inequality

$$
\begin{aligned}
\left\|\int_{t_{0}}^{t}\langle\nabla\rangle^{-1} e^{ \pm i(t-s)\langle\nabla\rangle} h(s) d s\right\|_{[Z]_{2}(I)} & \lesssim\left\|\int_{t_{0}}^{t}|t-s|^{-2 Z_{1}}\right\| h(s)\left\|_{B_{1 / T_{2}, 2}^{T_{3}}} d s\right\|_{L^{1 / T_{1}(I)}} \\
& \lesssim\|h\|_{[T]_{2}(I) .}
\end{aligned}
$$

This estimate can be translated in the time and the regularity exponents as

$$
Z \mapsto Z^{\prime}=Z+(b, 0, s), \quad T \mapsto T^{\prime}=T+(b, 0, s)
$$

for any $s \in \mathbb{R}$ and $b \in(-1 / 2,1 / 2)$, as long as $0<Z_{1}^{\prime}, T_{1}^{\prime}<1$. By the complex interpolation for those estimates and the standard Strichartz estimate, we obtain the desired estimate in the $\operatorname{case} \operatorname{str}^{\theta}(Z)=$ $\operatorname{str}^{\theta}(T)-2$ and $\operatorname{reg}^{\theta}(Z)=\operatorname{reg}^{\theta}(T)+2$. It is extended to the remaining cases (with inequality in these relations) by the Sobolev embedding.

The following interpolation is convenient for switching from some exponents to others:

Lemma 4.3. Let $Z, A, B, C \in[0,1] \times \mathbb{R}$ and $\theta \in[0,1]$. Assume that $A_{1}<Z_{1}<B_{1}$ and that either

(1) $\min \left(\operatorname{str}^{\theta}(A), \operatorname{str}^{\theta}(B), \operatorname{str}^{\theta}(C)\right) \geq \operatorname{str}^{\theta}(Z)$ and $\min \left(\operatorname{reg}^{\theta}(A), \operatorname{reg}^{\theta}(B)\right)>\operatorname{reg}^{\theta}(Z)$, or

(2) $\min \left(\operatorname{str}^{\theta}(A), \operatorname{str}^{\theta}(B)\right)>\operatorname{str}^{\theta}(Z)$ and $\min \left(\operatorname{reg}^{\theta}(A), \operatorname{reg}^{\theta}(B), \operatorname{reg}^{\theta}(C)\right) \geq \operatorname{reg}^{\theta}(Z)$.

Then there exist $\alpha, \beta, \gamma \in(0,1)$ such that $\alpha+\beta+\gamma=1$ and that, for all $q \in(0, \infty]$, we have the interpolation inequality

$$
\|u\|_{[Z]_{q}} \lesssim\|u\|_{[A]_{\infty}}^{\alpha}\|u\|_{[B]_{\infty}}^{\beta}\|u\|_{[C]_{\infty}}^{\gamma} .
$$

Proof. Since $A_{1}<Z_{1}<B_{1}$, for any $0<\theta_{2} \ll 1$ there exists $\theta_{1} \in(0,1)$ such that

$$
\left(1-\theta_{2}\right)\left(\left(1-\theta_{1}\right) A_{1}+\theta_{1} B_{1}\right)+\theta_{2} C_{1}=Z_{1} .
$$

Let $\widetilde{Z}:=\left(1-\theta_{2}\right)\left(\left(1-\theta_{1}\right) A+\theta_{1} B\right)+\theta_{2} C$. Then from the assumption we have

$$
\operatorname{str}^{\theta}(\tilde{Z}) \geq \operatorname{str}^{\theta}(Z), \quad \operatorname{reg}^{\theta}(\tilde{Z}) \geq \operatorname{reg}^{\theta}(Z),
$$

which imply $\tilde{Z}_{2} \geq Z_{2}$ and $\tilde{Z}_{3}-d \tilde{Z}_{2} \geq Z_{3}-d Z_{2}$, and so we have the Sobolev embedding $[\tilde{Z}]_{q} \subset[Z]_{q}$. In the first case, we have $\operatorname{reg}^{\theta}(\widetilde{Z})>\operatorname{reg}^{\theta}(Z)$ and so

$$
\left[\left[[A]_{\infty},[B]_{\infty}\right]_{\theta_{1}},[C]_{\infty}\right]_{\theta_{2}}=[\tilde{Z}]_{\infty} \subset[Z]_{q}
$$


The desired inequality follows from that for the complex interpolation.

It remains to prove the result under condition (2). By the real interpolation in the Besov space in $x$ and Hölder in $t$, we have for all $0<\delta \ll 1$,

$$
\|u\|_{[Z]_{q}} \lesssim\|u\|_{[Z+]_{\infty}}^{1 / 2}\|u\|_{[Z-]_{\infty}}^{1 / 2}, \quad Z^{ \pm}:=Z \pm \delta(1,0,1-2 \theta / d) .
$$

Let $0<\varepsilon \ll 1$ satisfy $\varepsilon\left(B_{1}-A_{1}\right)\left(1-\theta_{2}\right)=\delta$ and

$$
\widetilde{Z}^{ \pm}:=\left(1-\theta_{2}\right)\left(\left(1-\theta_{1} \mp \varepsilon\right) A+\left(\theta_{1} \pm \varepsilon\right) B\right)+\theta_{2} C .
$$

Then from the assumption and the definition of $Z^{ \pm}$and $\varepsilon$, we have

$$
\operatorname{str}^{\theta}\left(\widetilde{Z}^{ \pm}\right)>\operatorname{str}^{\theta}\left(Z^{ \pm}\right), \quad \operatorname{reg}^{\theta}\left(\tilde{Z}^{ \pm}\right) \geq \operatorname{reg}^{\theta}\left(Z^{ \pm}\right)=\operatorname{reg}^{\theta}(Z),
$$

when $\varepsilon>0$ is small. Hence we have the Sobolev embedding

$$
\left[\left[[A]_{\infty},[B]_{\infty}\right]_{\theta_{1} \pm \varepsilon},[C]_{\infty}\right]_{\theta_{2}}=\left[\tilde{Z}^{ \pm}\right]_{\infty} \subset\left[Z^{ \pm}\right]_{\infty},
$$

where the left-hand side is a nested complex interpolation space. Now the conclusion follows from the interpolation inequality.

Global perturbation of Strichartz norms. Now we fix a few particular exponents. Define $H, W, K$ by

$$
H:=\left(0, \frac{1}{2}, 1\right), \quad W:=\left(\frac{d-1}{2(d+1)}, W_{1}, \frac{1}{2}\right), \quad K:=\left(\frac{d}{2(d+2)}, K_{1}, \frac{1}{2}\right) .
$$

Then $[H]_{2}=L_{t}^{\infty} H_{x}^{1}$ is the energy space, while $W$ and $K$ are 1-admissible, diagonal and boundary exponents respectively for the wave $(\theta=0)$ and the Klein-Gordon $(\theta=1)$ equations:

$$
\begin{aligned}
& 1=\operatorname{reg}^{0}(H)=\operatorname{reg}^{1}(H)=\operatorname{reg}^{0}(W)=\operatorname{reg}^{1}(K), \\
& 0=\operatorname{str}^{0}(H)=\operatorname{str}^{1}(H)=\operatorname{str}^{0}(W)=\operatorname{str}^{1}(K) .
\end{aligned}
$$

Let $e q(u)$ denote the left-hand side of NLKG:

$$
e q(u):=u_{t t}-\Delta u+u-f^{\prime}(u) .
$$

Recall the convention $u \leftrightarrow \vec{u}$ (page 427) to switch to first-order equations. We will treat the $H^{1}$ critical case (1-28) together with the subcritical case. Since $f_{S}(u)$ is for small $|u|$ and $f_{L}(u)$ for large $|u|$, we may freely lower $p_{1}$ in (1-25) and raise $p_{2}$ in (1-26). Hence we assume (1-25) with

$$
2 \star-2=\frac{4}{d}<p_{1}<\frac{4(d+1)}{(d+2)(d-1)},
$$

and we assume either $d=1,(1-29)$, or (1-26), with

$$
\frac{4(d+1)}{d^{2}-d-1}<p_{2} \leq 2^{\star}-2 .
$$

Before the main perturbation lemma, we see that $[\mathrm{H}]_{2} \cap[\mathrm{W}]_{2} \cap[\mathrm{K}]_{2}$ is enough to bound the full Strichartz norms of the solutions. 
Lemma 4.4. Assume that $f$ satisfies (1-36). Let $Z, T$ and $U$ be 1-admissible. In the $2 D$ exponential case (1-29), let $\Theta \in(0,1)$. Then there exist a constant $C_{1}>0$ and a continuous function $C_{2}:(0, \infty) \rightarrow(0, \infty)$ such that for any interval $I$, any $t_{0} \in I$ and any $w(t, x)$, we have

$$
\|w\|_{[Z]_{2}(I)} \leq C_{1}\left\|\vec{w}\left(t_{0}\right)\right\|_{L_{x}^{2}}+C_{1}\|e q(w)\|_{\left(\left[T^{*(1)}\right]_{2}+\left[U^{*(1)}\right]_{2}\right)(I)}+C_{2}\left(\|w\|_{\left([H]_{2} \cap[W]_{2} \cap[K]_{2}\right)(I)}\right),
$$

provided, in the exponential case, that

$$
\sup _{t \in I} \kappa_{0}\|\nabla w\|_{L_{x}^{2}}^{2} \leq 4 \pi \Theta .
$$

We remark that (4-30) is needed only in the exponential case.

Proof. We may assume $\Theta>\frac{1}{2}$ without losing any generality. We introduce the new exponents $M^{\sharp}$ and $X$ by

$$
M^{\sharp}:=\frac{2}{p_{2}(d+1)}(1,1,0), \quad X:=\left(v, 0, v-v^{2}\right),
$$

with some $v \in(0,1 / 10)$ satisfying $\Theta<(1-v)^{2}$, where $M^{\sharp}$ is used only if $d \geq 2$ and $X$ only in the exponential case. In either case we have

$$
0>\operatorname{str}^{0}\left(M^{\sharp}\right), \quad 0>\operatorname{str}^{0}(X), \quad 1 \geq \operatorname{reg}^{0}\left(M^{\sharp}\right), \quad 1>\operatorname{reg}^{0}(X), \quad 0<M_{1}^{\#}, X_{1}<W_{1} .
$$

Hence by Lemma 4.3(1), we have

$$
\|w\|_{\left[M^{\sharp}\right]_{2}(I)}+\|w\|_{[X]_{2}(I)} \lesssim\|w\|_{\left([H]_{2} \cap[W]_{2} \cap[K]_{2}\right)(I)} .
$$

The Strichartz estimate gives

$\|w\|_{[Z]_{2}(I)}$

$$
\lesssim\left\|\vec{w}\left(t_{0}\right)\right\|_{L_{x}^{2}}+\|e q(w)\|_{\left(\left[T^{*(1)}\right]_{2}+\left[U^{*(1)}\right]_{2}\right)(I)}+\left\|f^{\prime}(w)\right\|_{\left(\left[K^{*(1)}\right]_{2}+\left[W^{*(1)}\right]_{2}+L_{t}^{1} L_{x}^{2}\right)(I)} .
$$

By the standard nonlinear estimate we have

$$
\left\|f_{S}^{\prime}(w)\right\|_{\left[K^{*(1)}\right]_{2}(I)} \lesssim\|w\|_{[K]_{2}(I)}\|w\|_{[K]_{0}(I)}^{4 / d},
$$

and in the subcritical/critical cases

$$
\left\|f_{L}^{\prime}(w)\right\|_{\left[W^{*(1)}\right]_{2}(I)} \lesssim\|w\|_{[W]_{2}(I)}\|w\|_{\left[M^{\sharp}\right]_{0}(I)}^{p_{2}} .
$$

In the exponential case, there are $\kappa>\kappa_{0}$ and $\mu>0$ such that

$$
\sup _{t \in I} \kappa\|w\|_{H_{\mu}^{1}}^{2} \leq 4 \pi \Theta^{\prime}
$$

where $\Theta^{\prime}:=\frac{1+\Theta}{2}<1$ and

$$
\|\varphi\|_{H_{\mu}^{1}}:=\|\nabla \varphi\|_{L_{x}^{2}}^{2}+\mu\|\varphi\|_{L_{x}^{2}}^{2}
$$

Then we have

$$
\left\|f_{L}^{\prime}(w)\right\|_{L_{x}^{2}} \lesssim\left\||w|\left(e^{\kappa|w|^{2}}-1\right)\right\|_{L_{x}^{2}} \lesssim\|w\|_{L_{x}^{\infty}}\left\|e^{\kappa|w|^{2}}-1\right\|_{L_{x}^{1}}^{1 / 2}\left\|e^{\kappa|w|^{2}}\right\|_{L_{x}^{\infty}}^{1 / 2},
$$


where the second factor is bounded by Trudinger-Moser:

$$
\left\|e^{\kappa|w|^{2}}-1\right\|_{L_{x}^{1}} \lesssim\|w\|_{L^{2}}^{2} /\left(1-\Theta^{\prime}\right)
$$

and the third factor is bounded by the following log-interpolation inequality [Ibrahim et al. 2007, Theorem 1.3]: for any $\alpha \in(0,1), \lambda>1 /(2 \pi \alpha)$ and $\mu>0$, there is $C>0$ such that

$$
\|\varphi\|_{L^{\infty}\left(\mathbb{R}^{2}\right)}^{2} \leq \lambda\|\varphi\|_{H_{\mu}^{1}\left(\mathbb{R}^{2}\right)}^{2}\left[C+\log \left(1+\|\varphi\|_{C^{\alpha}\left(\mathbb{R}^{2}\right)} /\|\varphi\|_{H_{\mu}^{1}\left(\mathbb{R}^{2}\right)}\right)\right],
$$

for any $\varphi \in H^{1} \cap C^{\alpha}\left(\mathbb{R}^{2}\right)$, where $C^{\alpha}=B_{\infty, \infty}^{\alpha}$ denotes the Hölder space. Plugging this with $\alpha:=v-v^{2}$ into the exponential, we get

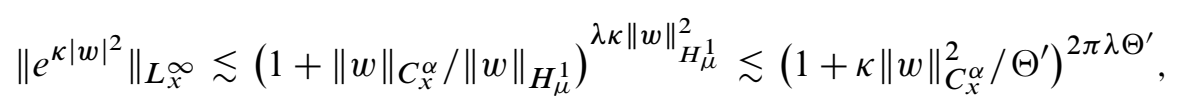

where $\lambda>0$ is chosen so that

$$
1<2 \pi \lambda \alpha, \quad\left(2 \pi \lambda \Theta^{\prime}+1\right) v=1 .
$$

Since $f_{L}$ vanishes for small $|u|$, we may assume $\|w\|_{C_{x}^{\alpha}} \gtrsim\|w\|_{L_{x}^{\infty}} \gtrsim 1$. Hence

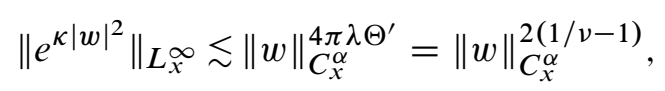

and plugging this into (4-39), we get

$$
\left\|f_{L}^{\prime}(w)\right\|_{L_{t}^{1} L_{x}^{2}} \lesssim\|w\|_{L_{t}^{1 / v} L_{x}^{\infty}}\|w\|_{L_{t}^{\infty} L_{x}^{2}}\|w\|_{L_{t}^{1 / \nu} C_{x}^{\alpha}}^{1 / v-1} \lesssim\|w\|_{[X]_{2}}^{1 / v}\|w\|_{[H]_{2}},
$$

which concludes the proof.

Lemma 4.5. Assume that $f$ satisfies (1-36). Let $Z, T, U$ and $V$ be 1-admissible and $\operatorname{reg}^{0}(V)=1$. In the exponential case (1-29), let $\Theta \in(0,1)$. Then there are continuous functions $\varepsilon_{0}, C_{0}:(0, \infty)^{2} \rightarrow$ $(0, \infty)$ such that the following holds: Let $I \subset \mathbb{R}$ be an interval, $t_{0} \in I$ and $\vec{u}, \vec{w} \in C\left(I ; L^{2}\left(\mathbb{R}^{d}\right)\right)$. Let $\overrightarrow{\gamma_{0}}=e^{i\langle\nabla\rangle\left(t-t_{0}\right)}(\vec{u}-\vec{w})\left(t_{0}\right)$ and assume that for some $A, B>0$ we have

$$
\begin{gathered}
\|\vec{u}\|_{L_{t}^{\infty}\left(I ; L_{x}^{2}\right)}+\|\vec{w}\|_{L_{t}^{\infty}\left(I ; L_{x}^{2}\right)} \leq A, \\
\|w\|_{[W]_{2}(I) \cap[K]_{2}(I)} \leq B, \\
\|(e q(u), e q(w))\|_{\left(\left[T^{*(1)}\right]_{2}+\left[U^{*(1)}\right]_{2}\right)(I)}+\left\|\gamma_{0}\right\|_{[V]_{\infty}(I)} \leq \varepsilon_{0}(A, B),
\end{gathered}
$$

and in the exponential case,

Then we have

$$
\sup _{t \in I} \kappa_{0} \max \left(\|\nabla u\|_{L_{x}^{2}}^{2},\|\nabla w\|_{L_{x}^{2}}^{2}\right) \leq 4 \pi \Theta
$$

$$
\|u\|_{[Z]_{2}(I)} \leq C_{0}(A, B) .
$$

Remark 4.6. (4-49) is needed only in the exponential case. The lemma remains valid in the lower critical case $p_{1}=4 / d=2 \star-2$, if we assume in addition that

$$
\left\|\gamma_{0}\right\|_{[K]_{0}(I)} \leq \varepsilon_{0}(A, B) .
$$

We will indicate the necessary modifications in the proof. 
Proof of Lemma 4.5. We restrict $p_{1}, p_{2}$ as in (4-27) and (4-28), without losing any generality. In the following, $C(\cdot, \ldots)$ denotes arbitrary positive constants which may depend continuously on the indicated parameters. Let $\delta \in(0,1)$ be a fixed small number, whose smallness will be specified by the following arguments. Let

$$
e:=e q(u)-e q(w), \quad \gamma:=u-w .
$$

Then we have the equation for the difference

$$
\ddot{\gamma}-\Delta \gamma+\gamma=f^{\prime}(w+\gamma)-f^{\prime}(w)-e, \quad \vec{\gamma}\left(t_{0}\right)=\vec{\gamma}_{0}\left(t_{0}\right) .
$$

First note that by Lemma 4.4, we have the full Strichartz norms on $w$.

Next we estimate the difference $u-w$ in the easier case $d \leq 4$. We define new exponents $S, L$ and a space $\mathscr{L}$ by

$$
\begin{aligned}
& {[S]_{0}:=L_{t}^{p_{1}+1} L_{x}^{2\left(p_{1}+1\right)}, \quad[L]_{0}:=L_{t}^{p_{2}+1} L_{x}^{2\left(p_{2}+1\right)},} \\
& \mathscr{X}:= \begin{cases}{[S]_{0}} & (d=1) \\
{[S]_{0} \cap[X]_{2}} & (1-29), \\
{[S]_{0} \cap[L]_{0}} & \text { (otherwise) } .\end{cases}
\end{aligned}
$$

Thanks to the restrictions (4-27) and (4-28), we have

$$
0>\operatorname{str}^{1}(S), \quad 0>\operatorname{str}^{0}(L), \quad 1>\operatorname{reg}^{1}(S), \quad 1>\operatorname{reg}^{0}(L) .
$$

Hence by Lemma 4.3(2) with $C:=V$, we get for some $\theta_{1}, \theta_{2} \in(0,1)$,

$$
\left\|\gamma_{0}\right\|_{\mathscr{X}(I)} \lesssim A^{1-\theta_{1}} \varepsilon_{0}^{\theta_{1}}+A^{1-\theta_{2}} \varepsilon_{0}^{\theta_{2}} \text {. }
$$

If $p_{1} \rightarrow 4 / d$, then $\operatorname{str}^{0}(S) \rightarrow 0$, and we would need the smallness in $[K]_{0}(I)$.

Since $w \in \mathscr{X}(I)$ by Lemma 4.4, there exists a partition of the right half of $I$ :

$$
t_{0}<t_{1}<\cdots<t_{n}, \quad I_{j}=\left(t_{j}, t_{j+1}\right), \quad I \cap\left(t_{0}, \infty\right)=\left(t_{0}, t_{n}\right)
$$

such that $n \leq C(A, B, \delta)$ and

$$
\|w\|_{\mathscr{L}\left(I_{j}\right)} \leq \delta \quad(j=0, \ldots, n-1)
$$

We omit the estimate on $I \cap\left(-\infty, t_{0}\right)$ since it is the same by symmetry.

Let $\gamma_{j}$ be the free solution defined by

$$
\vec{\gamma}_{j}:=e^{i\langle\nabla\rangle\left(t-t_{j}\right)} \vec{\gamma}\left(t_{j}\right)
$$

Then the Strichartz estimate applied to the equations of $\gamma$ and $\gamma_{j+1}$ implies

$$
\left\|\gamma-\gamma_{j}\right\|_{\mathscr{X}\left(I_{j}\right)}+\left\|\gamma_{j+1}-\gamma_{j}\right\|_{\mathscr{L}(\mathbb{R})} \lesssim\left\|f^{\prime}(w+\gamma)-f^{\prime}(w)\right\|_{L_{t}^{1} L_{x}^{2}\left(I_{j}\right)}+\|e\|_{\left(\left[U^{*(1)}\right]_{2}+\left[T^{*(1)}\right]_{2}\right)\left(I_{j}\right)} \text {. }
$$

The nonlinear difference is estimated as follows. For smaller $|u|$, we have by Hölder

$$
\left\|f_{S}^{\prime}(w+\gamma)-f_{S}^{\prime}(w)\right\|_{L_{t}^{1} L_{x}^{2}} \lesssim\|(w, \gamma)\|_{[S]_{0}}^{p_{1}}\|\gamma\|_{[S]_{0}},
$$


and for larger $|u|$ for $d \geq 2$ in the subcritical/critical cases,

$$
\left\|f_{L}^{\prime}(w+\gamma)-f_{L}^{\prime}(w)\right\|_{L_{t}^{1} L_{x}^{2}} \lesssim\|(w, \gamma)\|_{[L]_{0}}^{p_{2}}\|\gamma\|_{[L]_{0}} .
$$

If $d=1$, let $C(v)=\sup _{|u| \leq v}\left|f_{L}^{\prime \prime}(u)\right| /|u|^{p_{1}}$. Then we have

$$
\begin{aligned}
\left\|f_{L}^{\prime}(w+\gamma)-f_{L}^{\prime}(w)\right\|_{L_{t}^{1} L_{x}^{2}} & \lesssim C\left(\|w\|_{L_{t, x}^{\infty}}+\|\gamma\|_{L_{t, x}^{\infty}}\right)\|(w, \gamma)\|_{[S]_{0}}^{p_{1}}\|\gamma\|_{[S]_{0}} \\
& \lesssim C\left(\|(w, \gamma)\|_{L_{t}^{\infty} H_{x}^{1}}\right)\|(w, \gamma)\|_{[S]_{0}}^{p_{1}}\|\gamma\|_{[S]_{0}} .
\end{aligned}
$$

In the exponential case, there exist $\kappa>\kappa_{0}$ and $\mu>0$ such that (4-37). Let $w_{\theta}=w+\theta \gamma=(1-\theta) w+\theta u$ for $\theta \in[0,1]$. Then we have

$$
\kappa\left\|w_{\theta}\right\|_{H_{\mu}^{1}}^{2} \leq 4 \pi \Theta^{\prime},
$$

where $\Theta^{\prime}=\frac{1+\Theta}{2}$ and $H_{\mu}^{1}$ is defined in (4-38). In the same way as for (4-45), we obtain

$$
\begin{aligned}
\left\|f_{L}^{\prime}(w+\gamma)-f_{L}^{\prime}(w)\right\|_{L_{t}^{1} L_{x}^{2}} & \leq \int_{0}^{1}\left\|f_{L}^{\prime \prime}\left(w_{\theta}\right) \gamma\right\|_{L_{t}^{1} L_{x}^{2}} d \theta \lesssim \sup _{\theta \in[0,1]}\left\|w_{\theta}\right\|_{[H]_{2}}\left\|w_{\theta}\right\|_{[X]_{2}}^{1 / \nu-1}\|\gamma\|_{[X]_{2}} \\
& \lesssim A\|(w, \gamma)\|_{[X]_{2}}^{1 / \nu-1}\|\gamma\|_{[X]_{2}} .
\end{aligned}
$$

Thus in all cases, assuming

$$
\|\gamma\|_{\mathscr{L}\left(I_{j}\right)} \leq \delta \ll 1 \quad(j=0, \ldots, n-1)
$$

where the smallness depends on $A$ (and $\Theta$ ), we get

$$
\|\gamma\|_{\mathscr{L}\left(I_{j}\right)}+\left\|\gamma_{j+1}\right\|_{\mathscr{L}\left(t_{j+1}, t_{n}\right)} \leq C\left\|\gamma_{j}\right\|_{\mathscr{L}\left(t_{j}, t_{n}\right)}+\varepsilon_{0},
$$

for some absolute constant $C \geq 2$. Then by (4-56) and iteration in $j$ we get

$$
\|\gamma\|_{\mathscr{L}(I)} \lesssim(2 C)^{n}\left(A^{1-\theta_{1}} \varepsilon_{0}^{\theta_{1}}+A^{1-\theta_{2}} \varepsilon_{0}^{\theta_{2}}\right) \leq C(A, B)\left(\varepsilon_{0}^{\theta_{1}}+\varepsilon_{0}^{\theta_{2}}\right) .
$$

Choosing $\varepsilon_{0}(A, B)$ sufficiently small, we can make the last bound much smaller than $\delta$, and thus the assumption (4-65) is justified by continuity in $t$ and induction on $j$. Then repeating the estimate (4-60) once more, we can estimate the full Strichartz norms on $\gamma$, which implies also the bound on $u$.

Next we estimate the difference $u-w$ in the harder case $d \geq 5$, where we need the new exponents $\tilde{M}, M, \tilde{N}, N, R, Q, P$, and $Y$ defined by

$$
\begin{aligned}
M & =\frac{2}{d+1}\left[\frac{1}{p_{2}}(1+d, 0,0)-\frac{d-2}{4}(d,-1,0)\right], \\
\tilde{N} & =\frac{2}{d+1}\left[\left(\frac{1}{2}, \frac{d-1}{4}, 1\right)+\left(1-\frac{d-2}{4} p_{2}\right)(-d, 1,0)\right], \\
\tilde{M} & =M+\frac{2}{p_{2}(d+1)}(0,1 / d, 1), \quad N=\tilde{N}-\frac{2}{d+1}(0,1 / d, 1), \\
Q & =\frac{(1,2,2)}{p_{1}(d+1)}, \quad P=\frac{(4, d-1,4)}{2(d+1)}, \quad Y=\frac{(6, d+3,4)}{2(d+1)}, \\
R & =\left(\frac{(d+4)}{2(d+2)\left(p_{1}+1\right)}, R_{1}, \frac{1}{2}\right) .
\end{aligned}
$$


In the case $p_{2}>1$, we need another exponent

$$
\widehat{M}:=\tilde{M}+\frac{2\left(p_{2}-1\right)}{p_{2}(d+1)}(0,1 / d, 1),
$$

and if $p_{2} \leq 1$ then we put $\widehat{M}=\tilde{M}$. Note that $p_{1}<1$ under (4-27) for $d \leq 5$. Then we have the sharp Sobolev embedding

$$
[\widehat{M}]_{q} \subset[\tilde{M}]_{q} \subset[M]_{q}, \quad[\tilde{N}]_{q} \subset[N]_{q},
$$

and nonlinear and interpolation relations

$$
R+p_{1} R^{0}=K^{*(1)}, \quad R=(1-\alpha) W+\alpha K, \quad M^{\sharp}=(1-\beta) W^{0}+\beta R^{0},
$$

for some $\alpha, \beta \in(0,1)$, thanks to (4-27) and (4-28). $Y$ is a non-admissible exponent satisfying

$$
Y=\tilde{N}+p_{2} M=N+p_{2} \tilde{M}=P+p_{1} Q^{0}=P^{0}+p_{1} Q
$$

where the second and the last identities follow from $P_{3}=p_{1} Q_{3}, \tilde{N}_{3}=p_{2} \tilde{M}_{3}$, and the above sharp embeddings. If $p_{2}>1$, we have in addition

$$
Y=N+\widehat{M}+\left(p_{2}-1\right) M .
$$

These exponents satisfy (when $d \geq 5$ )

$$
\begin{gathered}
1=\operatorname{reg}^{0}(\tilde{N})=-\operatorname{reg}^{0}(Y) \geq \operatorname{reg}^{0}(\hat{M}), \quad 1>\operatorname{reg}^{1}(Q), \operatorname{reg}^{1}(P),-\operatorname{reg}^{1}(Y), \\
0>\operatorname{str}^{0}(\hat{M}), \operatorname{str}^{0}(\tilde{N}), \operatorname{str}^{1}(Q), \operatorname{str}^{1}(P), \\
\operatorname{str}^{0}(\tilde{N}) \leq \operatorname{str}^{0}(Y)-2, \quad \operatorname{str}^{1}(P)=\operatorname{str}^{1}(Y)-2, \\
0 \leq \hat{M}_{1}, \hat{M}_{2}, Q_{1}, Q_{2}, R_{1}<\frac{1}{2}, \quad 1<\operatorname{dec}^{0}(Y), \operatorname{dec}^{1}(Y), \\
Y_{2}<\frac{1}{2}+\frac{1}{d}, \quad \tilde{N}_{2}>\frac{1}{2}-\frac{1}{d-1}, \quad P_{2}>\frac{1}{2}-\frac{1}{d} .
\end{gathered}
$$

Moreover, $\operatorname{reg}^{0}(\hat{M})=1$ only if $p_{2}=2^{\star}-2=4 /(d-2)$. Lemma $4.3(1)$ implies that

$$
\|w\|_{\left([Q]_{2 p_{1}} \cap[\hat{M}]_{2} \cap[\tilde{M}]_{2 p_{2}}\right)(I)} \lesssim\|w\|_{\left([H]_{2} \cap[K]_{2} \cap[W]_{2}\right)(I)} \lesssim A+B .
$$

As before, we divide $I \cap\left(t_{0}, \infty\right)$ into $t_{0}<\cdots<t_{n}, n \leq C(A, B)$ such that

$$
\|w\|_{\left([Q]_{2 p_{1}} \cap[\hat{M}]_{2} \cap[\tilde{M}]_{2 p_{2}} \cap[K]_{2} \cap[W]_{2}\right)\left(I_{j}\right)} \leq \delta \ll 1 \quad(j=0, \ldots, n-1) .
$$

We also introduce the following spaces:

$$
\begin{aligned}
& \mathscr{y}_{0}:=[W]_{0} \cap[R]_{0}, \quad \tilde{y}:=[\tilde{N}]_{2} \cap[P]_{2}, \quad \text { y }:=[W]_{2} \cap[K]_{2}, \\
& y_{0}^{*}:=\left[W^{*(1)}\right]_{0}+\left[K^{*(1)}\right]_{0}, \quad y^{*}:=\left[W^{*(1)}\right]_{2}+\left[K^{*(1)}\right]_{2} .
\end{aligned}
$$

Our proof for $d \geq 5$ consists of three steps:

(1) We estimate $\gamma$ in $\mathscr{y}_{0}$, assuming it is bounded in some norm similar to (4-76). Here we can use the standard Strichartz because the estimates do not contain spatial derivative.

(2) We estimate $\gamma$ in $\widetilde{\mathscr{y}}$, under the same assumption on $\gamma$. Here we use the exotic Strichartz. 
(3) We estimate $u$ in $\mathscr{Y}$ by using the bounds in $[\tilde{N}]_{2} \cap[R]_{0}$. The assumption in the previous steps is justified once we get a better bound.

Actually we could skip the first step, by using interpolation in the last step to bound $[R]_{0}$ by the other norms. However, if $p_{1}=4 / d$ the lower critical power, then $R=K$ and the first step becomes necessary.

Assuming that

$$
\|\gamma\|_{\left([Q]_{2 p_{1}} \cap[\widehat{M}]_{2 p_{2}} \cap[R]_{0} \cap\left[M^{\sharp}\right]_{0}\right)\left(I_{j}\right)} \leq \delta \quad(j=0, \ldots, n-1),
$$

we have by Strichartz and Hölder (since $W^{0}$ and $R^{0}$ are $\frac{1}{2}$-admissible)

$$
\begin{aligned}
\left\|\gamma-\gamma_{j}\right\|_{9_{0}\left(I_{j}\right)}+\left\|\gamma_{j+1}-\gamma_{j}\right\|_{9_{0}(\mathbb{R})} & \lesssim\left\|f^{\prime}(w+\gamma)-f^{\prime}(w)\right\|_{9_{0}^{*}\left(I_{j}\right)}+\|e\|_{9 *\left(I_{j}\right)} \\
& \lesssim\|(w, \gamma)\|_{[R]_{0}\left(I_{j}\right)}^{p_{1}}\|\gamma\|_{[R]_{0}\left(I_{j}\right)}+\|(w, \gamma)\|_{\left[M^{\sharp}\right]_{0}\left(I_{j}\right)}^{p_{2}}\|\gamma\|_{[W]_{0}\left(I_{j}\right)}+\varepsilon_{0} \\
& \lesssim \delta^{p_{1}}\|\gamma\|_{9_{0}\left(I_{j}\right)}+\varepsilon_{0},
\end{aligned}
$$

where we used (4-76) and (4-78). By Lemma 4.3(2), we have

$$
\left\|\gamma_{0}\right\|_{9_{0}(I)} \lesssim A^{1-\theta_{3}} \varepsilon_{0}^{\theta_{3}}+A^{1-\theta_{4}} \varepsilon_{0}^{\theta_{4}}
$$

for some $\theta_{3}, \theta_{4} \in(0,1)$. Note that $\operatorname{str}^{1}(R) \rightarrow 0$ as $p_{1} \rightarrow 4 / d$, hence in the lower critical case we would need $\gamma_{0}$ to be small in $[K]_{0}$. By the same argument as for (4-67), we obtain

$$
\|\gamma\|_{\mathscr{y}_{0}(I)} \leq C(A, B)\left(\varepsilon_{0}^{\theta_{3}}+\varepsilon_{0}^{\theta_{4}}\right) \ll \delta .
$$

Next, still assuming (4-78), we have by the exotic Strichartz estimate,

$$
\left\|\gamma-\gamma_{j}\right\|_{\widetilde{\mathrm{y}}\left(I_{j}\right)}+\left\|\gamma_{j+1}-\gamma_{j}\right\|_{\widetilde{\mathrm{y}}(\mathbb{R})} \lesssim\left\|f^{\prime}(w+\gamma)-f^{\prime}(w)\right\|_{[Y]_{2}\left(I_{j}\right)}+\|e\|_{\mathrm{y}^{*}\left(I_{j}\right)},
$$

where the nonlinear difference is estimated by

$\left\|f_{L}^{\prime}(w+\gamma)-f_{L}^{\prime}(w)\right\|_{[Y]_{2}}$

$$
\lesssim\|(w, \gamma)\|_{[M]_{0}}^{p_{2}}\|\gamma\|_{[\tilde{N}]_{2}}+\|(w, \gamma)\|_{[\tilde{M}]_{2 p_{2}}}^{p_{2}}\|\gamma\|_{[N]_{0}}+\|(w, \gamma)\|_{[M]_{0}}^{p_{2}-1}\|(w, \gamma)\|_{[\hat{M}]_{2}}\|\gamma\|_{[N]_{0}},
$$

where the last term is for $p_{2}>1$ while the second last is for $p_{2} \leq 1$, and similarly

$$
\left\|f_{S}^{\prime}(w+\gamma)-f_{S}^{\prime}(w)\right\|_{[Y]_{2}} \lesssim\|(w, \gamma)\|_{[Q]_{0}}^{p_{1}}\|\gamma\|_{[P]_{2}}+\|(w, \gamma)\|_{[Q]_{2 p_{1}}}^{p_{1}}\|\gamma\|_{[P]_{0}} .
$$

Thus we obtain

$$
\left\|\gamma-\gamma_{j}\right\|_{\widetilde{y}_{\left(I_{j}\right)}}+\left\|\gamma_{j+1}-\gamma_{j}\right\|_{\widetilde{y}_{(\mathbb{R})}} \lesssim \delta^{p_{1}}\|\gamma\|_{\widetilde{y}_{\left(I_{j}\right)}}+\varepsilon_{0},
$$

where we used (4-76), (4-78), and the following embeddings in $x$

$$
[Q]_{2 p_{1}} \subset[Q]_{0}, \quad[P]_{2} \subset[P]_{0}, \quad[\hat{M}]_{2}+[\tilde{M}]_{2 p_{2}} \subset[M]_{0}, \quad[\tilde{N}]_{2} \subset[N]_{0} .
$$

By Lemma 4.3 and Strichartz, we have

$$
\begin{aligned}
\left\|\gamma_{0}\right\|_{[\tilde{N}]_{2}(I)} & \lesssim\left\|\gamma_{0}\right\|_{[H]_{2}(I) \cap[W]_{2}(I)}^{1-\theta_{5}}\left\|\gamma_{0}\right\|_{[M]_{0}(I)}^{\theta_{5}} \lesssim A^{1-\theta_{5}} \varepsilon_{0}^{\theta_{5}}, \\
\left\|\gamma_{0}\right\|_{[P]_{2}(I)} & \lesssim\left\|\gamma_{0}\right\|_{[H]_{2}(I) \cap[K]_{2}(I)}^{1-\theta_{6}}\left\|\gamma_{0}\right\|_{[M]_{0}(I)}^{\theta_{6}} \lesssim A^{1-\theta_{6}} \varepsilon_{0}^{\theta_{6}},
\end{aligned}
$$


for some $\theta_{5}, \theta_{6} \in(0,1)$. Note that $\operatorname{str}^{1}(P)$ is away from 0 as $p_{1} \rightarrow 4 / d$, and so $\theta_{5}, \theta_{6}$ are uniformly bounded from below. Thus by the same argument as for (4-67),

$$
\|\gamma\|_{\widetilde{y}_{(I)}} \leq C(A, B)\left(\varepsilon_{0}^{\theta_{5}}+\varepsilon_{0}^{\theta_{6}}\right) \ll \delta .
$$

Hence under the assumption (4-78) we have obtained

$$
\|\gamma\|_{[W]_{0}(I) \cap[R]_{0}(I) \cap[\tilde{N}]_{2}(I) \cap[P]_{2}(I)} \lesssim C(A, B) \sum_{k=3}^{6} \varepsilon_{0}^{\theta_{k}} \ll \delta .
$$

Finally by Strichartz, (4-76) and (4-78), we have

$$
\begin{aligned}
\|u\|_{9_{(}\left(I_{j}\right)} & \lesssim\left\|\vec{u}\left(t_{j}\right)\right\|_{L_{x}^{2}}+\left\|e q(u)+f^{\prime}(u)\right\|_{9 y^{*}\left(I_{j}\right)} \\
& \lesssim A+\varepsilon_{0}+\|u\|_{[R]_{0}\left(I_{j}\right)}^{p_{1}}\|u\|_{[R]_{2}\left(I_{j}\right)}+\|u\|_{\left[M^{\sharp}\right]_{0}\left(I_{j}\right)}^{p_{2}}\|u\|_{[W]_{2}\left(I_{j}\right)} \\
& \lesssim A+\varepsilon_{0}+\delta^{p_{1}}\|u\|_{\left.\mathscr{y}_{\left(I_{j}\right)}\right)}
\end{aligned}
$$

Hence we obtain

and so

$$
\|u\|_{\mathscr{Y}\left(I_{j}\right)} \lesssim A+\varepsilon_{0}
$$

$$
\|u\|_{9(I)} \lesssim n\left(A+\varepsilon_{0}\right) \leq C(A, B),
$$

which is extended to the full Strichartz norms by Lemma 4.4.

It remains to justify (4-78). By Lemma 4.3(2), we have

$$
\|\gamma\|_{[Q]_{2 p_{1}} \cap[\hat{M}]_{2} \cap[\tilde{M}]_{2 p_{2}}} \lesssim \sum_{k=7,8}\|\gamma\|_{[H]_{2} \cap[K]_{2} \cap[W]_{2}}^{1-\theta_{k}}\|\gamma\|_{[P]_{2} \cap[\tilde{N}]_{2}}^{\theta_{k}},
$$

for some $\theta_{7}, \theta_{8} \in(0,1)$. If $p_{1}=4 / d$, then we need to add $[K]_{0}$ to the last factor.

In either case, by (4-91), (4-76), (4-89), and (4-71), we obtain

$$
\|\gamma\|_{\left([Q]_{2 p_{1}} \cap[\hat{M}]_{2} \cap[\tilde{M}]_{2 p_{2}} \cap[R]_{0} \cap\left[M^{\sharp}\right]_{0}\right)\left(I_{j}\right)} \lesssim C(A, B) \varepsilon_{0}^{\theta},
$$

for some $\theta \in(0,1)$. By choosing $\varepsilon_{0}(A, B)$ sufficiently small, the last bound can be made much smaller than $\delta$. Then the assumption (4-78) is justified by continuity in $t$ and induction in $j$. Thus we have obtained the desired estimates.

\section{Profile decomposition}

In this section, following Bahouri, Gérard, Kenig, and Merle, we investigate behavior of general sequences of solutions, by asymptotic expansion into a series of transformation sequences of fixed spacetime functions, called profiles. This is the fundamental part for the construction of a critical element in the next section.

Linear profile decomposition. Here we give the Klein-Gordon version of Bahouri and Gérard's profile decomposition for the massless free wave equation. The only essential difference is that the massive equation does not commute with the scaling transforms, but the proof goes almost the same.

For simple presentation, we introduce some notation. For any triple $\left(t_{\circlearrowleft}^{\diamond}, x_{\circlearrowleft}^{\diamond}, h_{\circlearrowleft}^{\diamond}\right) \in \mathbb{R}^{1+d} \times(0, \infty)$ with arbitrary suffix $\odot$ and $\diamond$, let $\tau_{\circlearrowleft}^{\diamond}, T_{\odot}^{\diamond}$ and $\langle\nabla\rangle_{\circlearrowleft}^{\diamond}$ respectively denote the scaled time shift, the unitary 
and the self-adjoint operators in $L^{2}\left(\mathbb{R}^{d}\right)$, defined by

$$
\tau_{\odot}^{\diamond}=-\frac{t_{\odot}^{\diamond}}{h_{\odot}^{\diamond}}, \quad T_{\odot}^{\diamond} \varphi(x)=\left(h_{\odot}^{\diamond}\right)^{-d / 2} \varphi\left(\frac{x-x_{\odot}^{\diamond}}{h_{\odot}^{\diamond}}\right), \quad\langle\nabla\rangle_{\odot}^{\diamond}=\sqrt{-\Delta+\left(h_{\odot}^{\diamond}\right)^{2}} .
$$

We denote by $M \mathscr{C}$ the set of Fourier multipliers on $\mathbb{R}^{d}$ :

$$
M \mathscr{C}=\left\{\mathscr{F}^{-1} \tilde{\mu} \mathscr{F} \mid \tilde{\mu} \in C\left(\mathbb{R}^{d}\right) \text { and } \tilde{\mu}(x) \text { has a finite limit as }|x| \rightarrow \infty\right\} .
$$

(Practically we need only 1 and $|\nabla|\langle\nabla\rangle^{-1}$ in $M \bullet$ ). Also recall the correspondence $u \leftrightarrow \vec{u}$ defined on page 427.

Lemma 5.1 (Linear profile decomposition). Let $\vec{v}_{n}=e^{i\langle\nabla\rangle t} \vec{v}_{n}(0)$ be a sequence of free Klein-Gordon solutions with bounded $L_{x}^{2}$ norm. Then, possibly after replacing it with some subsequence, there exist $K \in\{0,1,2 \ldots, \infty\}$ and, for each integer $j \in[0, K), \varphi^{j} \in L^{2}\left(\mathbb{R}^{d}\right)$ and $\left\{\left(t_{n}^{j}, x_{n}^{j}, h_{n}^{j}\right)\right\}_{n \in \mathbb{N}} \subset \mathbb{R} \times \mathbb{R}^{d} \times$ $(0,1]$ satisfying the following. Define $\vec{v}_{n}^{j}$ and $\vec{w}_{n}^{k}$ for each $j<k \leq K$ by

$$
\vec{v}_{n}^{j}=e^{i\langle\nabla\rangle\left(t-t_{n}^{j}\right)} T_{n}^{j} \varphi^{j}, \quad \vec{v}_{n}=\sum_{j=0}^{k-1} \vec{v}_{n}^{j}+\vec{w}_{n}^{k}
$$

Then

$$
\lim _{k \rightarrow K} \varlimsup_{n \rightarrow \infty}\left\|\vec{w}_{n}^{k}\right\|_{L_{t}^{\infty}\left(\mathbb{R} ; B_{\infty, \infty}^{-d / 2}\left(\mathbb{R}^{d}\right)\right)}=0
$$

and for any Fourier multiplier $\mu \in M \mathscr{C}$, any $l<j<k \leq K$ and any $t \in \mathbb{R}$,

$$
\begin{gathered}
\lim _{n \rightarrow \infty}\left|\log \left(h_{n}^{l} / h_{n}^{j}\right)\right|+\frac{\left|t_{n}^{l}-t_{n}^{j}\right|+\left|x_{n}^{l}-x_{n}^{j}\right|}{h_{n}^{l}}=\infty, \\
\lim _{n \rightarrow \infty}\left\langle\mu \vec{v}_{n}^{l}(t) \mid \mu \vec{v}_{n}^{j}(t)\right\rangle_{L_{x}^{2}}=0=\lim _{n \rightarrow \infty}\left\langle\mu \vec{v}_{n}^{j}(t) \mid \mu \vec{w}_{n}^{k}(t)\right\rangle_{L_{x}^{2}} .
\end{gathered}
$$

Moreover, each sequence $\left\{h_{n}^{j}\right\}_{n \in \mathbb{N}}$ either goes to 0 or is identically 1 for all $n$.

We call such a sequence $\left\{\vec{v}_{n}^{j}\right\}_{n \in \mathbb{N}}$ a free concentrating wave for each $j$, and $\vec{w}_{n}^{k}$ the remainder. We say that $\left\{\left(t_{n}^{j}, x_{n}^{j}, h_{n}^{j}\right)\right\}_{n}$ and $\left\{\left(t_{n}^{k}, x_{n}^{k}, h_{n}^{k}\right)\right\}$ are orthogonal when (5-5) holds. Note that (5-6) implies

$$
\lim _{n \rightarrow \infty}\left[\left\|\vec{v}_{n}(t)\right\|_{L_{x}^{2}}^{2}-\sum_{j<k}\left\|\vec{v}_{n}^{j}(t)\right\|_{L_{x}^{2}}^{2}-\left\|\vec{w}_{n}^{k}\right\|_{L_{x}^{2}}^{2}\right]=0 .
$$

We remark that the case $h_{n}^{j} \rightarrow \infty$ is excluded by the presence of the mass, or more precisely by the use of inhomogeneous Besov norm for the remainder.

Proof. We introduce a Littlewood-Paley decomposition for the Besov norm. Let $\Lambda_{0}(x) \in \mathscr{S}\left(\mathbb{R}^{d}\right)$ such that its Fourier transform $\tilde{\Lambda}_{0}(\xi)=1$ for $|\xi| \leq 1$ and $\tilde{\Lambda}_{0}(\xi)=0$ for $|\xi| \geq 2$. Then we define $\Lambda_{k}(x)$ for any $k \in \mathbb{N}$ and $\Lambda_{(0)}(x)$ by the Fourier transforms

$$
\tilde{\Lambda}_{k}(\xi)=\tilde{\Lambda}_{0}\left(2^{-k} \xi\right)-\tilde{\Lambda}_{0}\left(2^{-k+1} \xi\right), \quad \tilde{\Lambda}_{(0)}=\tilde{\Lambda}_{0}(\xi)-\tilde{\Lambda}_{0}(2 \xi)
$$

Let

$$
v:=\varlimsup_{n \rightarrow \infty}\left\|\vec{v}_{n}\right\|_{L_{t}^{\infty} B_{\infty, \infty}^{-d / 2}} \sim \varlimsup_{n \rightarrow \infty} \sup _{t \in \mathbb{R}, x \in \mathbb{R}^{d}, k \geq 0} 2^{-k d / 2}\left|\Lambda_{k} * \vec{v}_{n}(t, x)\right|
$$


If $v=0$, we are done with $K=0$. Otherwise, there exists a sequence $\left(t_{n}, x_{n}, k_{n}\right)$ such that for large $n$

$$
2^{-k_{n} d / 2}\left|\Lambda_{k_{n}} * \vec{v}_{n}\left(t_{n}, x_{n}\right)\right| \geq v / 2 .
$$

Now we define $h_{n}$ and $\psi_{n}$ by

$$
h_{n}=2^{-k_{n}}, \quad \vec{v}_{n}\left(t_{n}, x\right)=T_{n} \psi_{n} .
$$

Since $\psi_{n}$ is bounded in $L_{x}^{2}$, it converges weakly to some $\psi$ in $L_{x}^{2}$, up to an extraction of a subsequence. Moreover,

$$
2^{-k_{n} d / 2}\left|\Lambda_{k_{n}} * \vec{v}_{n}\left(t_{n}, x_{n}\right)\right|= \begin{cases}\left|\Lambda_{0} * \psi_{n}(0)\right| & \left(k_{n}=0\right), \\ \left|\Lambda_{(0)} * \psi_{n}(0)\right| & \left(k_{n} \geq 1\right),\end{cases}
$$

and hence by the weak convergence and by Schwarz

$$
\|\psi\|_{L_{x}^{2}} \gtrsim\left|\left\langle\Lambda_{0} \mid \psi\right\rangle\right|+\left|\left\langle\Lambda_{(0)} \mid \psi\right\rangle\right| \geq v / 2 .
$$

If $h_{n} \rightarrow 0$, then we put $\left(t_{n}^{0}, x_{n}^{0}, h_{n}^{0}\right)=\left(t_{n}, x_{n}, h_{n}\right)$ and $\varphi^{0}=\psi$. Otherwise, we may assume, by extracting a subsequence, that $h_{n}$ converges to some $h_{\infty}>0$, and we put

$$
\left(t_{n}^{0}, x_{n}^{0}, h_{n}^{0}\right)=\left(t_{n}, x_{n}, 1\right), \quad \varphi^{0}=h_{\infty}^{-d / 2} \psi\left(x / h_{\infty}\right) .
$$

Then we have $T_{n} \psi-T_{n}^{0} \varphi^{0} \rightarrow 0$ strongly in $L_{x}^{2}$. Now we define $\vec{v}_{n}^{0}$ and $\vec{w}_{n}^{1}$ by

$$
\vec{v}_{n}^{0}=e^{i\langle\nabla\rangle\left(t-t_{n}^{0}\right)} T_{n}^{0} \varphi^{0}, \quad \vec{w}_{n}^{1}=\vec{v}_{n}-\vec{v}_{n}^{0} .
$$

Then $\left(T_{n}^{0}\right)^{-1} \vec{w}_{n}^{1}\left(t_{n}^{0}\right)=\left(T_{n}^{0}\right)^{-1} T_{n} \psi_{n}-\varphi^{0} \rightarrow 0$ weakly in $L^{2}$, and $\mu T_{n}^{0}=T_{n}^{0} \mu_{n}^{0}$, where $\mu_{n}^{0}$ denotes the Fourier multiplier whose symbol is the rescaling of $\mu$ 's, that is $\tilde{\mu}\left(\xi / h_{n}^{0}\right)$. By the definition of $\mathcal{M C}$, the symbol of $\mu_{n}^{0}$ converges, including the case $h_{n}^{0} \rightarrow 0$, so $\mu_{n}^{0}$ converges strongly in $L^{2}\left(\mathbb{R}^{d}\right)$ to some $\mu_{\infty}^{0}$. Hence

$$
\left\langle\mu \vec{v}_{n}^{0}\left(t_{n}^{0}\right) \mid \mu \vec{w}_{n}^{1}\left(t_{n}^{0}\right)\right\rangle_{L_{x}^{2}}=\left\langle\mu_{n}^{0} \varphi^{0} \mid \mu_{n}^{0}\left(T_{n}^{0}\right)^{-1} \vec{w}_{n}^{1}\left(t_{n}^{0}\right)\right\rangle_{L_{x}^{2}} \rightarrow 0 .
$$

The left-hand side is preserved in $t$, hence the above holds at any $t$. This is the decomposition for $k=1$.

Next we apply the same procedure to the sequence $\vec{w}_{n}^{1}$ in place of $\vec{v}_{n}$. Then either the Besov norm goes to 0 and $K=1$, or otherwise we find the next concentrating wave $\vec{v}_{n}^{1}$ and the remainder $\vec{w}_{n}^{2}$, such that for some $\left(t_{n}^{1}, x_{n}^{1}, h_{n}^{1}\right)$ and $\varphi^{1} \in L^{2}\left(\mathbb{R}^{d}\right)$,

$$
\vec{w}_{n}^{1}=\vec{v}_{n}^{1}+\vec{w}_{n}^{2}, \quad \vec{v}_{n}^{1}=e^{i\langle\nabla\rangle\left(t-t_{n}^{1}\right)} T_{n}^{1} \varphi^{1}, \quad\left\langle\mu \vec{v}_{n}^{1}(t) \mid \mu \vec{w}_{n}^{2}(t)\right\rangle_{L_{x}^{2}} \rightarrow 0,
$$

$\left(T_{n}^{1}\right)^{-1} \vec{w}_{n}^{2}\left(t_{n}^{1}\right) \rightarrow 0$ weakly in $L_{x}^{2}$ as $n \rightarrow \infty$, and

$$
\varlimsup_{n \rightarrow \infty}\left\|\vec{w}_{n}^{1}\right\|_{L_{t}^{\infty} B_{\infty, \infty}^{-d / 2}} \lesssim\left\|\varphi^{1}\right\|_{L^{2}} .
$$

Iterating the procedure, we obtain the desired decomposition. $L^{2}$ orthogonality implies $\left\|\varphi^{k}\right\|_{L_{x}^{2}} \rightarrow 0$ as $k \rightarrow \infty$, and then (5-18) (for general $k$ ) gives the decay of the remainder in the Besov norm.

It remains to prove the orthogonality (5-5) as well as (5-6). First we have

$$
\left\langle\mu \vec{v}_{n}^{l}(0) \mid \mu \vec{v}_{n}^{j}(0)\right\rangle=\left\langle e^{-i\langle\nabla\rangle t_{n}^{l}} T_{n}^{l} \mu_{n}^{l} \varphi^{l} \mid e^{-i\langle\nabla\rangle t_{n}^{j}} T_{n}^{j} \mu_{n}^{j} \varphi^{j}\right\rangle=\left\langle S_{n}^{j, l} \mu_{n}^{l} \varphi^{l} \mid \mu_{n}^{j} \varphi^{j}\right\rangle,
$$


where $\tilde{\mu}_{n}^{l}=\tilde{\mu}\left(\xi / h_{n}^{l}\right)$ as before, and $S_{n}^{j, l}$ is defined by

$$
S_{n}^{j, l}:=\left(T_{n}^{j}\right)^{-1} e^{i\langle\nabla\rangle\left(t_{n}^{j}-t_{n}^{l}\right)} T_{n}^{l}=e^{-i\langle\nabla\rangle_{n}^{j} t_{n}^{j, l}}\left(T_{n}^{j}\right)^{-1} T_{n}^{l}=e^{-i\langle\nabla\rangle_{n}^{j} t_{n}^{j, l}} T_{n}^{j, l},
$$

with the sequence

$$
\left(t_{n}^{j, l}, x_{n}^{j, l}, h_{n}^{j, l}\right):=\left(t_{n}^{l}-t_{n}^{j}, x_{n}^{l}-x_{n}^{j}, h_{n}^{l}\right) / h_{n}^{j} .
$$

Using the last formula in (5-20), (5-5), and the uniform time decay of $e^{i\langle\nabla\rangle_{n}^{j} t}: \mathscr{S} \rightarrow \mathscr{S}^{\prime}$, it is easy to see that $S_{n}^{j, l} \rightarrow 0$ weakly on $L_{x}^{2}$ as $n \rightarrow \infty$ for all $j<l$. Since $\tilde{\mu}_{n}^{l}=\tilde{\mu}\left(\xi / h_{n}^{l}\right)$ and $\tilde{\mu}_{n}^{j}$ are convergent, (5-19) also tends to 0 . Then we have also

$$
\left\langle\mu \vec{v}_{n}^{j}(t) \mid \mu \vec{w}_{n}^{k}(t)\right\rangle_{L_{x}^{2}}=\left\langle\mu \vec{v}_{n}^{j}(t) \mid \mu \vec{w}_{n}^{j+1}(t)-\sum_{m=j+1}^{k-1} \mu \vec{v}_{n}^{m}(t)\right\rangle_{L_{x}^{2}} \rightarrow 0
$$

thus we obtain (5-6). Now suppose that (5-5) fails. Then there exists a minimal $(l, j)$ breaking (5-5), with respect to the natural order

$$
\left(l_{1}, j_{1}\right) \leq\left(l_{2}, j_{2}\right) \Longleftrightarrow l_{1} \leq l_{2} \text { and } j_{1} \leq j_{2} .
$$

By extracting a subsequence, we may assume that $h_{n}^{l} \rightarrow h_{\infty}^{l}, \log \left(h_{n}^{l} / h_{n}^{j}\right),\left(t_{n}^{l}-t_{n}^{j}\right) / h_{n}^{l}$ and $\left(x_{n}^{l}-x_{n}^{j}\right) / h_{n}^{l}$ all converge. Now we inspect

$$
\left(T_{n}^{l}\right)^{-1} \vec{w}_{n}^{l+1}\left(t_{n}^{l}\right)=\sum_{m=l+1}^{j} S_{n}^{l, m} \varphi^{m}+S_{n}^{l, j}\left(T_{n}^{j}\right)^{-1} \vec{w}_{n}^{j+1}\left(t_{n}^{j}\right) .
$$

where $S_{n}^{l, j}$ converges strongly to a unitary operator, due to the convergence of $\left(t_{n}^{l, j}, x_{n}^{l, j}, h_{n}^{l, j}\right)$ and $h_{n}^{l}$. Since $S_{n}^{l, m} \rightarrow 0$ for $m<j$ and $\left(T_{n}^{j}\right)^{-1} \vec{w}_{n}^{j+1}\left(t_{n}^{j}\right) \rightarrow 0$ weakly in $L_{x}^{2}$, we deduce from the weak limit of (5-24) that $\varphi^{k}=0$, a contradiction. This proves the orthogonality (5-5).

Those free concentrating waves with scaling going to 0 are vanishing in any Besov space with less regularity. Hence in the subcritical case, we may freeze the scaling to 1 by regarding them as a part of remainder. Hence:

Corollary 5.2. Let $\vec{v}_{n}$ be a sequence of free Klein-Gordon solutions with bounded $L_{x}^{2}$ norm. Then, after replacing it with some subsequence, there exist $K \in\{0,1,2 \ldots, \infty\}$ and data $\varphi^{j} \in L^{2}\left(\mathbb{R}^{d}\right)$ and $\left\{\left(t_{n}^{j}, x_{n}^{j}\right)\right\}_{n \in \mathbb{N}} \subset \mathbb{R} \times \mathbb{R}^{d}$, for each integer $j \in[0, K)$, satisfying the following. Define $\vec{v}_{n}^{j}$ and $\vec{w}_{n}^{k}$ for each $j<k \leq K$ by

$$
\vec{v}_{n}^{j}=e^{i\langle\nabla\rangle\left(t-t_{n}^{j}\right)} \varphi^{j}\left(x-x_{n}^{j}\right), \quad \vec{v}_{n}=\sum_{j=0}^{k-1} \vec{v}_{n}^{j}+\vec{w}_{n}^{k} .
$$

Then, for any $s<-d / 2$, we have

$$
\lim _{k \rightarrow K} \varlimsup_{n \rightarrow \infty}\left\|\vec{w}_{n}^{k}\right\|_{L^{\infty}\left(\mathbb{R} ; B_{\infty, 1}^{s}\left(\mathbb{R}^{d}\right)\right)}=0
$$

and for any $\mu \in M \mathscr{C}$, any $l<j<k \leq K$ and any $t \in \mathbb{R}$,

$$
\begin{gathered}
\lim _{n \rightarrow \infty}\left\langle\mu \vec{v}_{n}^{l} \mid \mu \vec{v}_{n}^{j}\right\rangle_{L_{x}^{2}}^{2}=0=\lim _{n \rightarrow \infty}\left\langle\mu \vec{v}_{n}^{j} \mid \mu \vec{w}_{n}^{k}\right\rangle_{L_{x}^{2}}, \\
\lim _{n \rightarrow \infty}\left|t_{n}^{j}-t_{n}^{k}\right|+\left|x_{n}^{j}-x_{n}^{k}\right|=\infty .
\end{gathered}
$$


Orthogonality holds also for the nonlinear energy, which implies that the decomposition is closed in $\widetilde{\mathscr{K}}^{+}$. Recall the vector notation for the energy (page 427). We will use the following estimates for $1<p<\infty$ :

$$
\begin{gathered}
\left\|\left[|\nabla|-\langle\nabla\rangle_{n}\right] \varphi\right\|_{L_{x}^{p}} \lesssim h_{n}\left\|\left\langle\nabla / h_{n}\right\rangle^{-1} \varphi\right\|_{L_{x}^{p}}, \\
\left\|\left[|\nabla|^{-1}-\langle\nabla\rangle_{n}^{-1}\right] \varphi\right\|_{L_{x}^{p}} \lesssim\left\|\left\langle\nabla / h_{n}\right\rangle^{-2}|\nabla|^{-1} \varphi\right\|_{L_{x}^{p}} ;
\end{gathered}
$$

they hold uniformly for $0<h_{n} \leq 1$, by Mihlin's theorem on Fourier multipliers.

Lemma 5.3. Assume that $f$ satisfies (1-36). Let $\vec{v}_{n}$ be a sequence offree Klein-Gordon solutions satisfying $\vec{v}_{n}(0) \in \widetilde{\mathscr{K}}^{+}$and $\varlimsup_{n \rightarrow \infty} \widetilde{E}\left(\vec{v}_{n}(0)\right)<m$. Let $\vec{v}_{n}=\sum_{j<k} \vec{v}_{n}^{j}+\vec{w}_{n}^{k}$ be the linear profile decomposition given by Lemma 5.1. Except for the $H^{1}$ critical case (1-28), it may be given by Corollary 5.2 too. Then we have $\vec{v}_{n}^{j}(0) \in \widetilde{\mathscr{K}}^{+}$for large $n$ and all $j<K$, and

$$
\lim _{k \rightarrow K} \varlimsup_{n \rightarrow \infty}\left|\widetilde{E}\left(\vec{v}_{n}(0)\right)-\sum_{j<k} \widetilde{E}\left(\vec{v}_{n}^{j}(0)\right)-\widetilde{E}\left(\vec{w}_{n}^{k}(0)\right)\right|=0 .
$$

Moreover we have for all $j<K$

$$
0 \leq \varliminf_{n \rightarrow \infty} \widetilde{E}\left(\vec{v}_{n}^{j}(0)\right) \leq \varlimsup_{n \rightarrow \infty} \widetilde{E}\left(\vec{v}_{n}^{j}(0)\right) \leq \varlimsup_{n \rightarrow \infty} \widetilde{E}\left(\vec{v}_{n}(0)\right)
$$

where the last inequality becomes an equality only if $K=1$ and $\vec{w}_{n}^{1} \rightarrow 0$ in $L_{t}^{\infty} L_{x}^{2}$.

Proof. First we see that in the exponential case (1-29), all the profiles and remainders are in the subcritical regime. Since $\vec{v}_{n}(0) \in \widetilde{\mathscr{K}}^{+}$, Lemma 2.11 implies

$$
\left\|\nabla\langle\nabla\rangle^{-1} \operatorname{Re} \vec{v}_{n}(0)\right\|_{L_{x}^{2}}^{2}+\left\|\operatorname{Im} \vec{v}_{n}(0)\right\|_{L_{x}^{2}}^{2}<2 m \leq 4 \pi / \kappa_{0} .
$$

For any $\left(\theta_{0}, \ldots, \theta_{k}\right) \in \mathbb{C}^{1+k}$ satisfying $\|\theta\|_{L^{\infty}}=\max _{j}\left|\theta_{j}\right| \leq 1$, let

$$
v_{n}^{\theta}=\sum_{j<k} \theta_{j} v_{n}^{j}+\theta_{k} w_{n}^{k}
$$

Choosing $\mu=|\nabla|\langle\nabla\rangle^{-1} \in M \mathscr{C}$ in (5-27), we get

$$
\varlimsup_{n \rightarrow \infty} \sup _{t \in \mathbb{R}}\left\|\nabla v_{n}^{\theta}\right\|_{L_{x}^{2}}^{2} \leq \varlimsup_{n \rightarrow \infty}\left\|\nabla\langle\nabla\rangle^{-1} \vec{v}_{n}\right\|_{L_{x}^{2}}^{2}=: M<4 \pi / \kappa_{0} .
$$

Hence there exist $\kappa>\kappa_{0}$ and $q \in(1,2)$ such that $q \kappa M<4 \pi$.

Now we start proving (5-30) in all the cases. Since the linear version of (5-30) is given by Lemma 5.1, it suffices to show orthogonality in $F$, i.e.

$$
\lim _{k \rightarrow K} \varlimsup_{n \rightarrow \infty}\left|F\left(v_{n}(0)\right)-\sum_{j<k} F\left(v_{n}^{j}(0)\right)-F\left(w_{n}^{k}(0)\right)\right|=0 .
$$

For this we may neglect $w_{n}^{k}$, because by the decay in $B_{\infty, \infty}^{1-d / 2}$ and interpolation with the $H^{1}$ bound we have

$$
\lim _{k \rightarrow K} \varlimsup_{n \rightarrow \infty}\left\|w_{n}^{k}(0)\right\|_{L_{x}^{p}}=0 \quad\left(2<p \leq 2^{\star}\right) .
$$

In the exponential case, we deal with $w_{n}^{k}$ as follows. Let $v_{n}^{<k+\theta}=v_{n}-(1-\theta) w_{n}^{k}$ for $0 \leq \theta \leq 1$. Using 
the Hölder and Trudinger-Moser inequalities, we get

$$
\begin{aligned}
\left|F\left(v_{n}\right)-F\left(v_{n}^{<k}\right)\right| & \leq \int_{0}^{1} \int\left|f^{\prime}\left(v_{n}^{<k+\theta}\right) w_{n}^{k}\right| d x d \theta \leq \int_{0}^{1} d \theta\left\|e^{q \kappa\left|v_{n}^{<k+\theta}\right|^{2}}-1\right\|_{L_{x}^{1}}^{1 / q}\left\|w_{n}^{k}\right\|_{L_{x}^{q^{\prime}}} \\
& \leq \int_{0}^{1} d \theta\left[\frac{\left\|v_{n}^{<k+\theta}\right\|_{L_{x}^{2}}^{2}}{4 \pi-q \kappa M}\right]^{1 / q}\left\|w_{n}^{k}\right\|_{L_{x}^{q^{\prime}}}
\end{aligned}
$$

In the subcritical and exponential cases, it suffices to have the decay in $B_{\infty, 1}^{s}$ for all $s<1-d / 2$, which is given by Corollary 5.2. Thus in any case we are allowed to replace $v_{n}(0)$ by $v_{n}^{<k}(0)$ in (5-35).

Next we may discard those $j$ for which $\tau_{n}^{j}=-t_{n}^{j} / h_{n}^{j} \rightarrow \pm \infty$, since for any $p \in\left(2,2^{\star}\right]$ satisfying $1 / p=1 / 2-s / d$ with $s \in(0,1]$, we have

$$
\left\|v_{n}^{j}(0)\right\|_{L_{x}^{p}} \lesssim\left\|e^{-i\langle\nabla\rangle_{n}^{j} \tau_{n}^{j}}|\nabla|^{-s} \varphi^{j}\right\|_{L_{x}^{p}} \rightarrow 0 \quad(n \rightarrow \infty)
$$

by the decay of $e^{i\langle\nabla\rangle_{n}^{j} t}$ in $\mathscr{S} \rightarrow L^{p}$ as $|t| \rightarrow \infty$, which is uniform in $n$, and the Sobolev embedding $\dot{H}_{x}^{s} \subset L_{x}^{p}$.

So extracting a subsequence, we may assume that $\tau_{n}^{j}$ has a finite limit $\tau_{\infty}^{j}$ for all $j$. Let

$$
\psi^{j}:=\operatorname{Re} e^{-i\langle\nabla\rangle_{\infty}^{j} \tau_{\infty}^{j} \varphi^{j}} \in L_{x}^{2}\left(\mathbb{R}^{d}\right) .
$$

Then $v_{n}^{j}(0)-\langle\nabla\rangle^{-1} T_{n}^{j} \psi^{j} \rightarrow 0$ strongly in $H_{x}^{1}$, thus (5-35) has been reduced to

$$
\left|F\left(\sum_{j<k}\langle\nabla\rangle^{-1} T_{n}^{j} \psi^{j}\right)-\sum_{j<k} F\left(\langle\nabla\rangle^{-1} T_{n}^{j} \psi^{j}\right)\right| \rightarrow 0 .
$$

In the subcritical and exponential cases, if $h_{n}^{j} \rightarrow 0$ then $\langle\nabla\rangle^{-1} T_{n}^{j} \psi^{j} \rightarrow 0$ strongly in $L_{x}^{p}$ for $2 \leq p<$ $2^{\star}$, so it can be neglected. Hence we may assume that $h_{n}^{j} \equiv 1$. Then each $T_{n}^{j}\langle\nabla\rangle^{-1} \psi^{j}$ is getting away from the others as $n \rightarrow \infty$, and (5-40) follows.

In the critical case, if $h_{n}^{j} \rightarrow 0$ then we have by (5-29),

$$
\left\|\langle\nabla\rangle^{-1} T_{n}^{j} \psi^{j}-h_{n}^{j} T_{n}^{j}|\nabla|^{-1} \psi^{j}\right\|_{L_{x}^{2 \star}} \lesssim\left\|\left\langle\nabla / h_{n}^{j}\right\rangle^{-2}|\nabla|^{-1} \psi^{j}\right\|_{L_{x}^{2 \star}} \rightarrow 0 .
$$

Hence we may replace $\langle\nabla\rangle^{-1} T_{n}^{j} \psi^{j}$ in (5-40) by $h_{n}^{j} T_{n}^{j} \hat{\psi}^{j}$ for some $\hat{\psi}^{j} \in L^{2^{\star}}$, including the case $h_{n}^{j} \equiv 1$. Then we may further replace each $\hat{\psi}^{j}$ by

$$
\check{\psi}_{n}^{j}(x):=\hat{\psi}^{j}(x) \times \begin{cases}0 & \text { if there is } l<j \text { s.t. } h_{n}^{l}<h_{n}^{j} \text { and }\left(x-x_{n}^{j, l}\right) / h_{n}^{j, l} \in \operatorname{supp} \hat{\psi}^{l}, \\ 1 & \text { otherwise, }\end{cases}
$$

where $\left(x_{n}^{j, l}, h_{n}^{j, l}\right)$ is defined in (5-21), because (5-5) after the above reduction implies either $h_{n}^{j, l} \rightarrow 0$ or $\left|x_{n}^{j, l}\right| \rightarrow \infty$, and so $\breve{\psi}^{j} \rightarrow \hat{\psi}^{j}$ at almost every $x \in \mathbb{R}^{d}$ as $n \rightarrow \infty$, and strongly in $L_{x}^{2^{\star}}$ by the dominated convergence theorem. Now the decomposition is trivial

$$
F\left(\sum_{j<k} h_{n}^{j} T_{n}^{j} \check{\psi}_{n}^{j}\right)=\sum_{j<k} F\left(h_{n}^{j} T_{n}^{j} \check{\psi}_{n}^{j}\right),
$$

by the support property of $\breve{\psi}_{n}^{j}$. Thus we have obtained (5-35) and (5-30). 
By exactly the same argument, we obtain also

$$
\lim _{k \rightarrow K} \varlimsup_{n \rightarrow \infty}\left|\tilde{K}_{\alpha, \beta}\left(\vec{v}_{n}(0)\right)-\sum_{j<k} \widetilde{K}_{\alpha, \beta}\left(\vec{v}_{n}^{j}(0)\right)-\widetilde{K}_{\alpha, \beta}\left(\vec{w}_{n}^{k}(0)\right)\right|=0 .
$$

The remaining conclusions follow from the next lemma.

Lemma 5.4 (Decomposition in $\tilde{\mathscr{K}}^{+}$). Assume $f$ satisfies (1-36). Let $k \in \mathbb{N}$ and $\varphi_{0}, \ldots, \varphi_{k} \in H^{1}\left(\mathbb{R}^{d}\right)$. Assume that

$$
\begin{array}{ll}
\tilde{E}\left(\sum_{j=0}^{k} \varphi_{j}\right) \leq m-\delta, & \tilde{K}_{\alpha, \beta}\left(\sum_{j=0}^{k} \varphi_{j}\right) \geq-\varepsilon \\
\tilde{E}\left(\sum_{j=0}^{k} \varphi_{j}\right) \geq \sum_{j=0}^{k} \tilde{E}\left(\varphi_{j}\right)-\varepsilon, & \tilde{K}_{\alpha, \beta}\left(\sum_{j=0}^{k} \varphi_{j}\right) \leq \sum_{j=0}^{k} \tilde{K}_{\alpha, \beta}\left(\varphi_{j}\right)+\varepsilon
\end{array}
$$

for some $(\alpha, \beta)$ in (1-16) and some $\delta, \varepsilon>0$ satisfying $\varepsilon(1+2 / \bar{\mu})<\delta$. Then $\tilde{\varphi}_{j} \in \tilde{\mathscr{K}}^{+}$for all $j=0, \ldots, k$, i.e. $0 \leq \widetilde{E}\left(\varphi_{j}\right)<m$ and $\widetilde{K}_{\alpha, \beta}\left(\varphi_{j}\right) \geq 0$ for all $(\alpha, \beta)$ in $(1-16)$.

Proof. Let $\psi_{j}=\operatorname{Re}\langle\nabla\rangle^{-1} \varphi_{j}$ and suppose that $\tilde{K}\left(\varphi_{l}\right)<0$ for some $l$. Then $K\left(\psi_{l}\right) \leq \tilde{K}\left(\varphi_{l}\right)<0$ and so $H\left(\psi_{l}\right) \geq m$. Since $H$ is non-negative,

$$
\begin{aligned}
& m \leq \sum_{j=0}^{k} H\left(\psi_{j}\right) \leq \sum_{j=0}^{k}\left[H\left(\psi_{j}\right)+H^{Q}\left(\operatorname{Im}\langle\nabla\rangle^{-1} \varphi_{j}\right)\right]=\sum_{j=0}^{k}\left[\widetilde{E}\left(\varphi_{j}\right)-\tilde{K}\left(\varphi_{j}\right) / \bar{\mu}\right] \\
& \leq \widetilde{E}\left(\sum_{j=0}^{k} \varphi_{j}\right)-\widetilde{K}\left(\sum_{j=0}^{k} \varphi_{j}\right) / \bar{\mu}+\varepsilon(1+1 / \bar{\mu})<m
\end{aligned}
$$

where $H^{Q}$ denotes the quadratic part of $H$. Hence $K\left(\psi_{j}\right) \geq 0$ for all $j$, and so

$$
\widetilde{E}\left(\varphi_{j}\right) \geq J\left(\psi_{j}\right)=H\left(\psi_{j}\right)+K\left(\psi_{j}\right) / \bar{\mu} \geq 0
$$

Nonlinear profile decomposition. The next step is to construct a similar decomposition for the nonlinear solutions with the same initial data.

First we construct a nonlinear profile corresponding to a free concentrating wave. Let $\vec{v}_{n}$ be a free concentrating wave for a sequence $\left(t_{n}, x_{n}, h_{n}\right) \in \mathbb{R} \times \mathbb{R}^{d} \times(0,1]$,

$$
\left(i \partial_{t}+\langle\nabla\rangle\right) \vec{v}_{n}=0, \quad \vec{v}_{n}\left(t_{n}\right)=T_{n} \psi, \quad \psi(x) \in L^{2},
$$

satisfying $\vec{v}_{n}(0) \in \widetilde{\mathscr{K}}^{+}$. Here we use Lemma 5.1 only in the $H^{1}$ critical case, and Corollary 5.2 in the subcritical and exponential cases. Hence $h_{n} \rightarrow 0$ can happen only in the critical case, otherwise $h_{n} \equiv 1$. Let $u_{n}$ be the nonlinear solution with the same initial data

$$
\left(i \partial_{t}+\langle\nabla\rangle\right) \vec{u}_{n}=f^{\prime}\left(u_{n}\right), \quad \vec{u}_{n}(0)=\vec{v}_{n}(0) \in \widetilde{\mathscr{K}}^{+},
$$

which may be local in time. Next we define $\vec{V}_{n}$ and $\vec{U}_{n}$ by undoing the transforms

$$
\vec{v}_{n}=T_{n} \vec{V}_{n}\left(\left(t-t_{n}\right) / h_{n}\right), \quad \vec{u}_{n}=T_{n} \vec{U}_{n}\left(\left(t-t_{n}\right) / h_{n}\right) .
$$


Then they satisfy the rescaled equations

$$
\vec{V}_{n}=e^{i t\langle\nabla\rangle_{n}} \psi, \quad \vec{U}_{n}=\vec{V}_{n}-i \int_{\tau_{n}}^{t} e^{i(t-s)\langle\nabla\rangle_{n}} f^{\prime}\left(\operatorname{Re}\langle\nabla\rangle_{n}^{-1} \vec{U}_{n}\right) d s,
$$

where $\tau_{n}=-t_{n} / h_{n}$. Extracting a subsequence, we may assume convergence:

$$
h_{n} \rightarrow h_{\infty} \in[0,1], \quad \tau_{n} \rightarrow \tau_{\infty} \in[-\infty, \infty] .
$$

Then the limit equations are naturally given by

$$
\vec{V}_{\infty}=e^{i t\langle\nabla\rangle_{\infty}} \psi, \quad \vec{U}_{\infty}=\vec{V}_{\infty}-i \int_{\tau_{\infty}}^{t} e^{i(t-s)\langle\nabla\rangle_{\infty}} f^{\prime}\left(\hat{U}_{\infty}\right) d s
$$

where $\hat{U}_{\infty}$ is defined by

$$
\hat{U}_{\infty}:=\operatorname{Re}\langle\nabla\rangle_{\infty}^{-1} \vec{U}_{\infty}= \begin{cases}\operatorname{Re}\langle\nabla\rangle^{-1} \vec{U}_{\infty} & \left(h_{\infty}=1\right), \\ \operatorname{Re}|\nabla|^{-1} \vec{U}_{\infty} & \left(h_{\infty}=0\right) .\end{cases}
$$

The unique existence of a local solution $\vec{U}_{\infty}$ around $t=\tau_{\infty}$ is known in all cases, including $h_{\infty}=0$ and $\tau_{\infty}= \pm \infty$ (the latter corresponding to the existence of the wave operators), by using the standard iteration with the Strichartz estimate. In the exponential case, it requires that $\vec{U}_{\infty}$ is in the subcritical regime in the Trudinger-Moser inequality. It is guaranteed by Lemma 5.3, because $\vec{V}_{\infty}(t) \in \widetilde{\mathscr{K}}^{+}$for $t$ close to $\tau_{\infty}$, and so $\vec{U}_{\infty}(t) \in \widetilde{\mathscr{K}}^{+}$for all $t$ in its existence interval.

$\vec{U}_{\infty}$ on the maximal existence interval is called the nonlinear profile associated with the free concentrating wave $\vec{v}_{n}$. The nonlinear concentrating wave $\vec{u}_{(n)}$ associated with $\vec{v}_{n}$ is defined by

$$
\vec{u}_{(n)}=T_{n} \vec{U}_{\infty}\left(\left(t-t_{n}\right) / h_{n}\right) .
$$

If $h_{\infty}=1$ then $u_{(n)}$ solves NLKG. If $h_{\infty}=0$ then it solves

$$
\left(\partial_{t}^{2}-\Delta+1\right) u_{(n)}=\left(i \partial_{t}+\langle\nabla\rangle\right) \vec{u}_{(n)}=(\langle\nabla\rangle-|\nabla|) \vec{u}_{(n)}+f^{\prime}\left(|\nabla|^{-1}\langle\nabla\rangle u_{(n)}\right) .
$$

The existence time of $u_{(n)}$ may be finite and even go to 0 , but at least we have

$$
\begin{aligned}
\left\|\vec{u}_{n}(0)-\vec{u}_{(n)}(0)\right\|_{L_{x}^{2}} & =\left\|\vec{V}_{n}\left(\tau_{n}\right)-\vec{U}_{\infty}\left(\tau_{n}\right)\right\|_{L_{x}^{2}} \\
& \leq\left\|\vec{V}_{n}\left(\tau_{n}\right)-\vec{V}_{\infty}\left(\tau_{n}\right)\right\|_{L_{x}^{2}}+\left\|\vec{V}_{\infty}\left(\tau_{n}\right)-\vec{U}_{\infty}\left(\tau_{n}\right)\right\|_{L_{x}^{2}} \rightarrow 0 .
\end{aligned}
$$

Let $u_{n}$ be a sequence of (local) solutions of NLKG in $\mathscr{K}^{+}$around $t=0$, and let $v_{n}$ be the sequence of the free solutions with the same initial data. We consider the linear profile decomposition given by Lemma 5.1 or 5.2:

$$
\vec{v}_{n}=\sum_{j=0}^{k-1} \vec{v}_{n}^{j}+\vec{w}_{n}^{k}, \quad \vec{v}_{n}^{j}=e^{i\langle\nabla\rangle\left(t-t_{n}^{j}\right)} T_{n}^{j} \varphi^{j}
$$

With each free concentrating wave $\left\{\vec{v}_{n}^{j}\right\}_{n \in \mathbb{N}}$, we associate the nonlinear concentrating wave $\left\{\vec{u}_{(n)}^{j}\right\}_{n \in \mathbb{N}}$. A nonlinear profile decomposition of $u_{n}$ is given by

$$
\vec{u}_{(n)}^{<k}:=\sum_{j=0}^{k-1} \vec{u}_{(n)}^{j} .
$$


We are going to prove that $\vec{u}_{(n)}^{<k}$ is a good approximation for $\vec{u}_{n}$, provided that each nonlinear profile has finite global Strichartz norm (in Lemma 5.6). Now we define the Strichartz norms for the profile decomposition, using the notation from page 428. Let $S T$ and $S T^{*}$ be the function spaces on $\mathbb{R}^{1+d}$ defined by

$$
S T=[W]_{2} \cap[K]_{2}, \quad S T^{*}=\left[W^{*(1)}\right]_{2}+\left[K^{*(1)}\right]_{2}+L_{t}^{1} L_{x}^{2},
$$

where the exponents $W$ and $K$ as well as their duals are as defined in (4-24) and (4-9). The Strichartz norm for the nonlinear profile depends on the scaling $h_{\infty}^{\diamond}$ for any suffix $\diamond$ :

$$
S T_{\infty}^{\diamond}:= \begin{cases}{[W]_{2} \cap[K]_{2}} & \left(h_{\infty}^{\diamond}=1\right) \\ {[W]_{2}^{\bullet}} & \left(h_{\infty}^{\diamond}=0\right)\end{cases}
$$

In other words, we take the scaling invariant part if $h_{n}^{\diamond} \rightarrow+0$, which can happen only in the $H^{1}$ critical case. The following estimate will be convenient in treating the concentrating case: For any $S \in[0,1] \times\left[0, \frac{1}{2}\right] \times[0,1]$ we have

$$
\left\|u_{(n)}\right\|_{[S]_{2}(\mathbb{R})} \lesssim\left(h_{n}\right)^{1-\operatorname{reg}^{0}(S)}\left\|\widehat{U}_{\infty}\right\|_{[S]_{2}^{\bullet(\mathbb{R})}},
$$

where $\hat{U}_{\infty}$ is as defined in (5-53). Indeed, using $\dot{B}_{p, 2}^{0} \subset L^{p}$ with $p=1 / S_{2} \geq 2$ in the lower frequencies, we have

$$
\begin{aligned}
& \left\|u_{(n)}\right\|_{[S]_{2}} \lesssim\left\||\nabla|^{-S_{3}}\langle\nabla\rangle^{S_{3}} u_{(n)}\right\|_{[S]_{2}} \\
& \sim\left(h_{n}\right)^{1-\mathrm{reg}^{0}(S)}\left\|\operatorname{Re}|\nabla|^{-S_{3}}\langle\nabla\rangle_{n}^{S_{3}-1} \vec{U}_{\infty}^{j}\right\|_{[S]_{2}^{*}} \lesssim\left(h_{n}\right)^{1-\mathrm{reg}^{0}(S)}\left\|\hat{U}_{\infty}^{j}\right\|_{[S]_{2}^{\bullet}} .
\end{aligned}
$$

Concerning the orthogonality in the Strichartz norms, we have:

Lemma 5.5. Assume that $f$ satisfies (1-36). Suppose that in the nonlinear profile decomposition (5-58) we have

$$
\left\|\widehat{U}_{\infty}^{j}\right\|_{S T_{\infty}^{j}(\mathbb{R})}+\left\|\vec{U}_{\infty}^{j}\right\|_{L_{t}^{\infty} L_{x}^{2}(\mathbb{R})}<\infty
$$

for each $j<K$. Then, for any finite interval $I$, any $j<K$ and any $k \leq K$, we have

$$
\begin{aligned}
\varlimsup_{n \rightarrow \infty}\left\|u_{(n)}^{j}\right\|_{S T(I)} & \lesssim\left\|\widehat{U}_{\infty}^{j}\right\|_{S T_{\infty}^{j}(\mathbb{R})} \\
\varlimsup_{n \rightarrow \infty}\left\|u_{(n)}^{<k}\right\|_{S T(I)}^{2} & \lesssim \varlimsup_{n \rightarrow \infty} \sum_{j<k}\left\|u_{(n)}^{j}\right\|_{S T(I)}^{2},
\end{aligned}
$$

where the implicit constants do not depend on $I, j$ or $k$. We also have

$$
\lim _{n \rightarrow \infty}\left\|f^{\prime}\left(u_{(n)}^{<k}\right)-\sum_{j<k} f^{\prime}\left(\left(\langle\nabla\rangle_{\infty}^{j}\right)^{-1}\langle\nabla\rangle u_{(n)}^{j}\right)\right\|_{S T^{*}(I)}=0 .
$$

Proof. First note that if $h_{\infty}^{j}=1$ then $u_{(n)}^{j}$ is just a sequence of space-time translations of $\hat{U}_{\infty}^{j}$. In particular, (5-64) is trivial in that case.

Next we prove (5-64) in the case $h_{\infty}^{j}=0$, which is only in the $H^{1}$ critical case. For the moment we drop the superscript $j$. For the $[W]_{2}$ part, (5-61) gives us

$$
\left\|u_{(n)}\right\|_{[W]_{2}(I)} \lesssim\left\|\hat{U}_{\infty}\right\|_{[W]_{2}^{\bullet(\mathbb{R})}}=\left\|\hat{U}_{\infty}\right\|_{S T_{\infty}^{j}(\mathbb{R})} .
$$


For the $[K]_{2}$ part, let $V$ be the following interpolation between $H$ and $W$

$$
V:=\frac{1}{d+2} H+\frac{d+1}{d+2} W=K+\frac{(-1,0,1)}{2(d+2)} .
$$

Then using Hölder in $t$ and (5-61) together with $\operatorname{reg}^{0}(K)=(d+1) /(d+2)$, we get

$$
\left\|u_{(n)}\right\|_{[K]_{2}(I)} \lesssim\left\|u_{(n)}\right\|_{\left[V^{\frac{1}{2}}\right]_{2}(I)}|I|^{\frac{1}{2(d+2)}} \lesssim\left(h_{n}\right)^{\frac{1}{2(d+2)}}\left\|\widehat{U}_{\infty}\right\|_{[V]_{2}^{\mathbf{R}(\mathbb{R})}}|I|^{\frac{1}{2(d+2)}} \rightarrow 0,
$$

as $n \rightarrow \infty$. Thus we have proved (5-64).

Next we prove (5-65) in the subcritical and exponential cases. Define $\hat{U}_{\infty, R}^{j}, u_{(n), R}^{j}$ for $R \gg 1$ and $u_{(n), R}^{<k}$ by

$$
\hat{U}_{\infty, R}^{j}=\chi_{R}(t, x) \hat{U}_{\infty}^{j}, \quad u_{(n), R}^{j}=T_{n}^{j} \hat{U}_{\infty, R}^{j}\left(t-t_{n}^{j}\right), \quad u_{(n), R}^{<k}=\sum_{j<k} u_{(n), R}^{j},
$$

where $\chi_{R}$ is the cut-off defined in (1-23). Then we have

$$
\left\|u_{(n)}^{<k}-u_{(n), R}^{<k}\right\|_{S T(\mathbb{R})} \leq \sum_{j<k}\left\|\left(1-\chi_{R}(t, x)\right) \hat{U}_{\infty}^{j}\right\|_{S T(\mathbb{R})} \rightarrow 0, \quad(R \rightarrow+0)
$$

so we may replace $u_{(n)}^{<k}$ by $u_{(n), R}^{<k}$. Let $\delta_{m}^{l}$ denote the difference operator

$$
\delta_{m}^{l} \varphi(x)=\varphi\left(x-2^{-m} e_{l}\right)-\varphi(x),
$$

where $e_{l}$ denotes the $l$-th unit vector in $\mathbb{R}^{d}$. Each Besov norm in $S T$ is equivalent to

$$
\sum_{l=1}^{d}\left\|\sum_{j<k} 2^{s m} \delta_{m}^{l} u_{(n), R}^{j}\right\|_{L_{t}^{p} \ell_{m \geq 0}^{2} L_{x}^{q}}+\left\|\sum_{j<k} u_{(n), R}^{j}\right\|_{L_{t}^{p} L_{x}^{q}},
$$

where $(1 / p, 1 / q, s)=W$ or $K$. (5-28) implies that each $\operatorname{supp} u_{(n), R}^{j}$ is away from the others at least by distance 2 for large $n$, and then $\operatorname{supp} \delta_{m}^{l} u_{(n), R}^{j}$ are also disjoint for $j<k$ at each $l, m$. Hence the first norm in (5-73) equals

$$
\left\|2^{s m} \delta_{m}^{l} u_{(n), R}^{j}\right\|_{L_{t}^{p} \ell_{m \geq 0}^{2} L_{x}^{q} \ell_{j<k}^{2}} \leq\left\|2^{s m} \delta_{m}^{l} u_{(n), R}^{j}\right\|_{\ell_{j<k}^{2} L_{t}^{p} \ell_{m \geq 0}^{2} L_{x}^{q}} \lesssim\left\|u_{(n), R}^{j}\right\|_{\ell_{j<k}^{2} L_{t}^{p} B_{q, 2}^{s}},
$$

where the first inequality is by Minkowski. Thus we have obtained (5-65) in the subcritical and exponential cases.

Next we prove (5-65) in the $H^{1}$ critical case. For the nonlinear concentrating waves with $h_{\infty}^{j}=1$, the above argument works. For those with $h_{\infty}^{j}=0$, the $K$ component is vanishing by (5-69). Hence it suffices to estimate $[W]_{2}$ in the case all $h_{n}^{j}$ tend to 0 as $j \rightarrow \infty$. Using that $W_{3}=\frac{1}{2} \in(0,1)$, we have

$$
\left\|u_{(n)}^{<k}\right\|_{[W]_{2}(\mathbb{R})} \lesssim\left\||\nabla|^{-1}\langle\nabla\rangle u_{(n)}^{<k}\right\|_{[W]_{2}^{\bullet(\mathbb{R})}}=\left\|\operatorname{Re}|\nabla|^{-1} \vec{u}_{(n)}^{<k}\right\|_{[W]_{2}^{\bullet(\mathbb{R})}} \sim\left\|\sum_{j<k} \check{u}_{n, m}^{j, l}\right\|_{L_{t}^{p} \ell_{m \in \mathbb{Z}}^{2} L_{x}^{q}}
$$

where we have put $(1 / p, 1 / q, s)=W$ and

$$
\check{u}_{n, m}^{j, l}:=2^{s m} \delta_{m}^{l} h_{n}^{j} T_{n}^{j} \hat{U}_{\infty}^{j}\left(\left(t-t_{n}^{j}\right) / h_{n}^{j}\right),
$$


where $\delta_{m}^{l}$ is the difference operator defined in (5-72). For $R \gg 1$, let

$$
\check{u}_{n, m, R}^{j, l}(t, x):=\left\{\begin{array}{cc}
\chi_{h_{n}^{j} R}\left(t-t_{n}^{j}, x-x_{n}^{j}\right) \check{u}_{n, m}^{j, l}(t, x) & \left(\left|m-\log _{2} h_{n}^{j}\right| \leq R\right) \\
0 & \left(\left|m-\log _{2} h_{n}^{j}\right|>R\right),
\end{array}\right.
$$

where $\chi_{*}$ is as in (1-23). Then by the same computation as for (5-61), we have

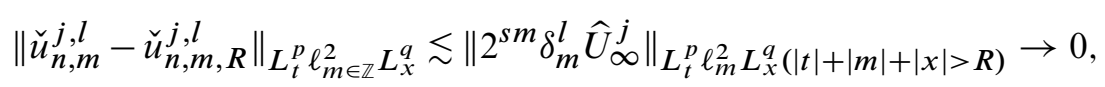

as $R \rightarrow \infty$ uniformly in $n$. Hence we may replace $\check{u}_{n, m}^{j, l}$ by $\check{u}_{n, m, R}^{j, l}$ in (5-75). The orthogonality (5-5) implies that $\left\{\operatorname{supp}_{(t, m, x)} \check{u}_{n, m, R}^{j, l}\right\}_{j<k}$ becomes mutually disjoint for large $n$. Then arguing as in (5-74), we obtain (5-65).

To prove (5-66) in the subcritical and exponential cases is easier than (5-65), because after the smooth cut-off, we have for large $n$

$$
f^{\prime}\left(u_{(n), R}^{<k}\right)=\sum_{j<k} f^{\prime}\left(u_{(n), R}^{j}\right)
$$

Note that the $u_{(n)}^{j} \in S T$ implies that the full Strichartz norms are finite by Lemma 4.4. The error for $f^{\prime}\left(u_{(n)}^{<k}\right)$ coming from the cut-off is small in $S T^{*}$ by (4-61)-(4-64) if $d \leq 4$. When $d \geq 5$, the difference estimates in the proof of Lemma 4.5 are not sufficient because they control only the exotic norm $Y$. In order to estimate the difference in the admissible dual norm $S T^{*}(I)$, we introduce the new exponents

$$
H_{\varepsilon}:=\left(\varepsilon^{2}, \frac{1-\varepsilon}{2}, 0\right), \quad W_{\varepsilon}:=W-p_{2} \varepsilon(d,-1,0), \quad M_{\varepsilon}^{\sharp}:=M^{\sharp}+\varepsilon(d,-1,0),
$$

where $W$ and $M^{\sharp}$ were defined in (4-24) and (4-31), and $\varepsilon \in\left(0, p_{1}\right)$ is fixed small enough to have

$$
\begin{aligned}
& \operatorname{str}^{0}\left(H_{\varepsilon}\right), \operatorname{str}^{0}\left(M_{\varepsilon}^{\sharp}\right), \operatorname{str}^{0}\left(W_{\varepsilon}\right)<0, \quad \operatorname{reg}^{0}\left(H_{\varepsilon}\right)<1, \\
& \operatorname{reg}^{0}\left(W_{\varepsilon}\right)=\operatorname{reg}^{0}(W)=1, \quad \operatorname{reg}^{0}\left(M_{\varepsilon}^{\sharp}\right)=\operatorname{reg}^{0}\left(M^{\sharp}\right) \leq 1, \\
& W_{\varepsilon}+p_{2} M_{\varepsilon}^{\sharp}=W+p_{2} M^{\sharp}=W^{*(1)} .
\end{aligned}
$$

Then we have, for any $u$ and $v$,

$$
\left\|f_{S}^{\prime}(u)-f_{S}^{\prime}(v)\right\|_{L_{t}^{1} L_{x}^{2}(I)} \lesssim|I|^{1-\varepsilon^{2}}\|u-v\|_{\left[H_{\varepsilon}\right]_{0}(I)}\left(\|u\|_{L_{t}^{\infty} L_{x}^{2}(I)}+\|v\|_{L_{t}^{\infty} L_{x}^{2}(I)}\right)^{\varepsilon},
$$

because $\left|f_{S}^{\prime}(u)-f_{S}^{\prime}(v)\right| \lesssim|u-v|(|u|+|v|)^{\varepsilon}$. For large $u$, we have if $p_{2} \geq 1$,

$\left\|f_{L}^{\prime}(u)-f_{L}^{\prime}(v)\right\|_{\left[W^{*(1)}\right]_{2}}$

$$
\lesssim\|u\|_{\left[M_{\varepsilon}^{\sharp}\right]_{0}}^{p_{2}}\|u-v\|_{\left[W_{\varepsilon}\right]_{2}}+\|u-v\|_{\left[M_{\varepsilon}^{\sharp}\right]_{0}}\left(\|u\|_{\left[M_{\varepsilon}^{\sharp}\right]_{0}}+\|v\|_{\left[M_{\varepsilon}^{\sharp}\right]_{0}}\right)^{p_{2}-1}\|v\|_{\left[W_{\varepsilon}\right]_{2}},
$$

and if $p_{2}<1$,

$$
\left\|f_{L}^{\prime}(u)-f_{L}^{\prime}(v)\right\|_{\left[W_{\varepsilon}^{*(1)}\right]_{2}} \lesssim\|u\|_{\left[M_{\varepsilon}^{\sharp}\right]_{0}}^{p_{2}}\|u-v\|_{\left[W_{\varepsilon}\right]_{2}}+\|u-v\|_{\left[M_{\varepsilon}^{\sharp}\right]_{0}}^{p_{2}}\|v\|_{\left[W_{\varepsilon}\right]_{2}} .
$$

The latter estimate is not Lipschitz in $u-v$, but suffices for our purpose here. ${ }^{4}$ Thus we obtain (5-66) in the subcritical and exponential cases.

\footnotetext{
${ }^{4}$ The situation is different from the long-time iteration in the previous section, where we needed the exotic Strichartz estimate in order to get the Lipschitz estimate for the iteration along the numerous time intervals.
} 
It remains to prove (5-66) in the $H^{1}$ critical case, where we need further cut-off to get a disjoint sum. First we see that each $u_{(n)}^{j}$ in $u_{(n)}^{<k}$ may be replaced with

$$
u_{\langle n\rangle}^{j}:=\left(\langle\nabla\rangle_{\infty}^{j}\right)^{-1}\langle\nabla\rangle u_{(n)}^{j}=h_{n}^{j} T_{n}^{j} \hat{U}_{\infty}^{j}\left(\left(t-t_{n}^{j}\right) / h_{n}^{j}\right) .
$$

For the moment we drop the superscript $j$. Let $p_{2}=4 /(d-2)$ and $h_{\infty}=0$. If $d \leq 4$, then we have by using (4-62) and (5-29)

$$
\begin{aligned}
\left\|f^{\prime}\left(u_{(n)}\right)-f^{\prime}\left(u_{\langle n\rangle}\right)\right\|_{L_{t}^{1} L_{x}^{2}(\mathbb{R})} & \lesssim\left\|u_{\langle n\rangle}\right\|_{[L]_{0}(\mathbb{R})}^{p_{2}}\left\|u_{(n)}-u_{\langle n\rangle}\right\|_{[L]_{0}(\mathbb{R})} \\
& \sim\left\|\widehat{U}_{\infty}\right\|_{[L]_{0}(\mathbb{R})}^{p_{2}}\left\|\left[|\nabla|\langle\nabla\rangle_{n}^{-1}-1\right] \widehat{U}_{\infty}\right\|_{[L]_{0}(\mathbb{R})} \\
& \lesssim\left\|\widehat{U}_{\infty}\right\|_{[L]_{0}(\mathbb{R})}^{p_{2}}\left\|\left\langle\nabla / h_{n}\right\rangle^{-2} \widehat{U}_{\infty}\right\|_{[L]_{0}(\mathbb{R})} \rightarrow 0
\end{aligned}
$$

since $\widehat{U}_{\infty} \in[H]_{2}^{\bullet} \cap[W]_{2}^{\bullet} \subset[L]_{0}$ by the homogeneous version of Lemma 4.3(1).

If $d \geq 5$, we introduce a new exponent

$$
G:=\frac{d-2}{d+2}\left(\frac{1}{d+1}, \frac{d+3}{2(d+1)}, 0\right) .
$$

Then $\operatorname{reg}^{0}(G)=1, \operatorname{str}^{0}(G)<0$ and

$$
\left(2^{\star}-1\right) G=W^{*(1)}-\frac{(1,0,1)}{2} .
$$

Hence

$$
\begin{aligned}
\left\|f^{\prime}\left(u_{(n)}\right)-f^{\prime}\left(u_{\langle n\rangle}\right)\right\|_{\left[W^{*(1)}\right]_{2}(I)} & \\
& \lesssim\left\|f^{\prime}\left(u_{(n)}\right)-f^{\prime}\left(u_{\langle n\rangle}\right)\right\|_{\left[W^{*(1)}\right]_{2}^{\bullet(\mathbb{R})}}+|I|^{1 / 2}\left\|f^{\prime}\left(u_{(n)}\right)-f^{\prime}\left(u_{\langle n\rangle}\right)\right\|_{\left[\left(2^{\star}-1\right) G\right]_{0}(I)},
\end{aligned}
$$

where the first term on the right is dominated by (the homogeneous version of (5-83)-(5-84))

$$
\begin{aligned}
\left\|u_{\langle n\rangle}\right\|_{\left[M_{\varepsilon}^{\sharp}\right]_{0}(\mathbb{R})}^{p_{2}}\left\|u_{(n)}-u_{\langle n\rangle}\right\|_{\left[W_{\varepsilon}\right]_{2}^{\bullet(\mathbb{R})}}+\left\|u_{(n)}-u_{\langle n\rangle}\right\|_{\left[M_{\varepsilon}^{\sharp}\right]_{0}(\mathbb{R})}^{\theta}\left\|\left(u_{\langle n\rangle}, u_{(n)}\right)\right\|_{\left[W_{\varepsilon}\right]_{2}^{\bullet}(\mathbb{R})}^{p_{2}-\theta} & \\
& \lesssim\left\|\widehat{U}_{\infty}\right\|_{\left[M_{\varepsilon}^{\sharp}\right]_{0}(\mathbb{R})}^{p_{2}}\left\|\left\langle\nabla / h_{n}\right\rangle^{-2} \widehat{U}_{\infty}\right\|_{\left[W_{\varepsilon}\right]_{2}^{\bullet}(\mathbb{R})}+\left\|\left\langle\nabla / h_{n}\right\rangle^{-2} \widehat{U}_{\infty}\right\|_{\left[M_{\varepsilon}^{\sharp}\right]_{0}(\mathbb{R})}^{\theta}\left\|\widehat{U}_{\infty}\right\|_{\left[W_{\varepsilon}\right]_{2}^{\bullet}(\mathbb{R})}^{p_{2}-\theta},
\end{aligned}
$$

where $\theta:=\min \left(p_{2}, 1\right)$. The right-hand side goes to 0 , since $\widehat{U}_{\infty} \in[H]_{2}^{\bullet} \cap\left[W_{\varepsilon}\right]_{2}^{\bullet} \subset\left[M_{\varepsilon}^{\sharp}\right]_{0}$ by the homogeneous version of Lemma 4.3(1). Similarly, the last term in (5-89) is bounded by

$$
\left\|u_{\langle n\rangle}\right\|_{[G]_{0}(\mathbb{R})}^{p_{2}}\left\|u_{(n)}-u_{\langle n\rangle}\right\|_{[G]_{0}(\mathbb{R})} \sim\left\|\widehat{U}_{\infty}\right\|_{[G]_{0}(\mathbb{R})}^{p_{2}}\left\|\left\langle\nabla / h_{n}\right\rangle^{-2} \widehat{U}_{\infty}\right\|_{[G]_{0}(\mathbb{R})} \rightarrow 0
$$

Thus it suffices to show

$$
\left\|f^{\prime}\left(\sum_{j<k} u_{\langle n\rangle}^{j}\right)-\sum_{j<k} f^{\prime}\left(u_{\langle n\rangle}^{j}\right)\right\|_{S T^{*}(I)} \rightarrow 0 .
$$

Now we define $\widehat{U}_{n, R}^{j}$ for any $R \gg 1$ by

$$
\hat{U}_{n, R}^{j}(t, x)=\chi_{R}(t, x) \hat{U}_{\infty}^{j}(t, x) \prod\left\{\left(1-\chi_{h_{n}^{j, l} R}\right)\left(t-t_{n}^{j, l}, x-x_{n}^{j, l}\right) \mid 1 \leq l<k, h_{n}^{l} R<h_{n}^{j}\right\},
$$

where $\chi_{R}$ and $\left(t_{n}^{j, l}, x_{n}^{j, l}, h_{n}^{j, l}\right)$ are as defined respectively in (1-23) and (5-21). Then $\widehat{U}_{n, R}^{j}$ is uniformly bounded in $[H]_{2}^{\bullet}(\mathbb{R}) \cap[W]_{2}^{\bullet}(\mathbb{R})$, and

$$
\widehat{U}_{n, R}^{j} \rightarrow \chi_{R} \widehat{U}_{\infty}^{j} \quad \text { in }\left[M^{\sharp}\right]_{0}(\mathbb{R}) \text { as } n \rightarrow \infty,
$$


because either $h_{n}^{j, l} \rightarrow 0$ or $\left|t_{n}^{j, l}\right|+\left|x_{n}^{j, l}\right| \rightarrow \infty$ by the orthogonality (5-5). Then by the homogeneous version of Lemma 4.3(2), it converges also in $[L]_{0}(\mathbb{R})$ (if $\left.d \leq 4\right),\left[W_{\varepsilon}\right]_{2}^{\bullet}(\mathbb{R})$ and $\left[M_{\varepsilon}^{\sharp}\right]_{0}(\mathbb{R})$. Moreover, we have $\chi_{R} \hat{U}_{\infty}^{j} \rightarrow \hat{U}_{\infty}^{j}$ as $R \rightarrow \infty$ in the same spaces.

Hence we may replace $u_{\langle n\rangle}^{j}$ by

$$
u_{\langle n\rangle, R}^{j}:=h_{n}^{j} T_{n}^{j} \widehat{U}_{n, R}^{j}\left(\left(t-t_{n}^{j}\right) / h_{n}^{j}\right),
$$

and then we get the desired result, since $\left\{\operatorname{supp}_{(t, x)} u_{\langle n\rangle, R}^{j}\right\}_{j<k}$ are mutually disjoint for large $n$, and so

$$
f^{\prime}\left(\sum_{j<k} u_{\langle n\rangle, R}^{j}\right)=\sum_{j<k} f^{\prime}\left(u_{\langle n\rangle, R}^{j}\right),
$$

which concludes the proof of (5-66).

The next lemma is the conclusion of this section.

Lemma 5.6. Assume that $f$ satisfies (1-36). Let $u_{n}$ be a sequence of local solutions of NLKG around $t=0$ in $\mathscr{K}^{+}$satisfying $\varlimsup_{n \rightarrow \infty} E\left(u_{n}\right)<m$. Suppose that in its nonlinear profile decomposition (5-58), every nonlinear profile $\vec{U}_{\infty}^{j}$ has finite global Strichartz and energy norms, i.e.

$$
\left\|\hat{U}_{\infty}^{j}\right\|_{S T_{\infty}^{j}(\mathbb{R})}+\left\|\vec{U}_{\infty}^{j}\right\|_{L_{t}^{\infty} L_{x}^{2}(\mathbb{R})}<\infty .
$$

Then $u_{n}$ is bounded for large $n$ in the Strichartz and the energy norms, i.e.

$$
\varlimsup_{n \rightarrow \infty}\left\|u_{n}\right\|_{S T(\mathbb{R})}+\left\|\vec{u}_{n}\right\|_{L_{t}^{\infty} L_{x}^{2}(\mathbb{R})}<\infty .
$$

Proof. We will apply the perturbation lemma to $u_{(n)}^{<k}+w_{n}^{k}$ as an approximate solution. First observe that

$$
\left\|\vec{u}_{n}(0)-\vec{u}_{(n)}^{<k}(0)-w_{n}^{k}(0)\right\|_{L_{x}^{2}} \leq \sum_{j<k}\left\|\vec{v}_{n}^{j}(0)-\vec{u}_{(n)}^{j}(0)\right\|_{L_{x}^{2}}=o(1)
$$

and

$$
\left\|\vec{u}_{n}(0)\right\|_{L^{2}}^{2}=\left\|\vec{v}_{n}\right\|_{L_{x}^{2}}^{2} \geq \sum_{j<k}\left\|\vec{v}_{n}^{j}\right\|_{L_{x}^{2}}^{2}+o(1)=\sum_{j<k}\left\|\vec{u}_{(n)}^{j}(0)\right\|_{L_{x}^{2}}^{2}+o(1),
$$

where $o(1) \rightarrow 0$ as $n \rightarrow \infty$. Hence except for a finite set $J \subset \mathbb{N}$, the energy of $u_{(n)}^{j}$ with $j \notin J$ is smaller than the iteration threshold, which implies

$$
\left\|u_{(n)}^{j}\right\|_{S T(\mathbb{R})} \lesssim\left\|\vec{u}_{(n)}^{j}(0)\right\|_{L_{x}^{2}} \quad(j \notin J) .
$$

Combining (5-65), (5-64), (5-99) and (5-98), we obtain, for any finite interval $I$,

$$
\sup _{k} \varlimsup_{n \rightarrow \infty}\left\|u_{(n)}^{<k}\right\|_{S T(I)}^{2} \lesssim \sum_{j \in J}\left\|\hat{U}_{\infty}^{j}\right\|_{S T_{\infty}^{j}}^{2}+\varlimsup_{n \rightarrow \infty}\left\|\vec{u}_{n}(0)\right\|_{L_{x}^{2}}^{2}<\infty .
$$

The equation of $u_{(n)}^{<k}$ is given by

$$
e q\left(u_{(n)}^{<k}\right)=\sum_{j<k}\left(\langle\nabla\rangle-\langle\nabla\rangle_{\infty}^{j}\right) \vec{u}_{(n)}^{j}+f^{\prime}\left(u_{(n)}^{<k}\right)-\sum_{j<k} f^{\prime}\left(u_{\langle n\rangle}^{j}\right),
$$


where $u_{\langle n\rangle}^{j}=\left(\langle\nabla\rangle_{\infty}^{j}\right)^{-1}\langle\nabla\rangle u_{(n)}^{j}$ as before. The nonlinear part goes to 0 by (5-66), while the linear part vanishes if $h_{\infty}^{j}=1$, and is dominated if $h_{\infty}^{j}=0$ by

$$
\begin{aligned}
\left\|(\langle\nabla\rangle-|\nabla|) \vec{u}_{(n)}^{j}\right\|_{L_{t}^{1} L_{x}^{2}(I)} & \lesssim|I|\left\|\langle\nabla\rangle^{-1} \vec{u}_{(n)}^{j}\right\|_{L_{t}^{\infty} L_{x}^{2}(\mathbb{R})} \\
& \sim|I|\left\|\left\langle\nabla / h_{n}^{j}\right\rangle^{-1} \vec{U}_{\infty}^{j}\right\|_{L_{t}^{\infty} L_{x}^{2}(\mathbb{R})} \rightarrow 0 \quad(n \rightarrow \infty),
\end{aligned}
$$

by continuity in $t$ for bounded $t$, and by the scattering of $\hat{U}_{\infty}^{j}$ for $|t| \rightarrow \infty$, which follows from $\left\|\widehat{U}_{\infty}^{j}\right\|_{[W]_{2}^{\bullet(\mathbb{R})}}<\infty$. Hence Lemma 4.4 gives for any 1 -admissible $Z$

$$
\sup _{k} \varlimsup_{n \rightarrow \infty}\left\|u_{(n)}^{<k}\right\|_{[Z]_{2}(\mathbb{R})}<\infty .
$$

On the other hand, by Lemma 4.3 we can extend the smallness of $w_{n}^{k}$ from $L_{t}^{\infty} B_{\infty, \infty}^{s}$ to the other spaces that we need for the nonlinear difference estimates, those being $[S]_{0},[L]_{0},[X]_{2},\left[H_{\varepsilon}\right]_{0},\left[M_{\varepsilon}^{\sharp}\right]_{0}$, and $\left[W_{\varepsilon}\right]_{2}$, depending on $d$ and $f$. In addition, in the exponential case (1-29), Lemmas 5.3 and 2.11 imply that $u_{(n)}^{<k}$ and $w_{n}^{k}$ are both in the subcritical regime for the Trudinger-Moser inequality. Putting them together with the above bounds on $u_{(n)}^{<k}$ in the nonlinear difference estimates (4-61)-(4-64) or (5-82)-(5-84), we get

$$
\lim _{k \rightarrow K} \varlimsup_{n \rightarrow \infty}\left\|f^{\prime}\left(u_{(n)}^{<k}+w_{n}^{k}\right)-f^{\prime}\left(u_{(n)}^{<k}\right)\right\|_{S T^{*}(I)}=0
$$

and so

$$
\lim _{k \rightarrow K} \varlimsup_{n \rightarrow \infty}\left\|e q\left(u_{(n)}^{<k}+w_{n}^{k}\right)\right\|_{S T^{*}(I)}=0 .
$$

Hence for $k$ sufficiently close to $K$ and $n$ large enough, the true solution $u_{n}$ and the approximate solution $u_{(n)}^{<k}+w_{n}^{k}$ satisfy all the assumptions of the perturbation Lemma 4.5. Hence $u_{n}$ is bounded in global Strichartz norms for large $n$.

\section{Extraction of a critical element}

In this section, we prove that if uniform global Strichartz bound fails strictly below the variational threshold $m$, then we have a global solution in $\mathcal{K}^{+}$with infinite Strichartz norm and with the minimal energy, which is called a critical element.

Let $E^{\star}$ be the threshold for the uniform Strichartz bound. More precisely,

$$
E^{\star}:=\sup \{A>0 \mid S(A)<\infty\},
$$

where $S(A)$ denotes the supremum of $\|u\|_{S T(I)}$ for any strong solution $u$ in $\mathcal{K}^{+}$on any interval $I$ satisfying $E(u) \leq A$.

The small energy scattering tells us $E^{\star}>0$, and the presence of the ground state tells us $E^{\star} \leq m$, at least in the subcritical case, and also in the other cases if we allow complex-valued solutions, because the stationary solutions with different masses yield standing wave solutions of the original NLKG. Anyway, we are going to prove $E^{\star} \geq m$ by contradiction.

We remark that there is an alternative threshold:

$$
E_{F S}^{\star}:=\sup \left\{\begin{array}{l|l}
A>0 & \begin{array}{l}
\text { if } u \text { is a solution in } \mathscr{K}^{+} \text {of } \mathrm{NLKG} \\
\text { with } E(u) \leq A, \text { then }\|u\|_{S T(\mathbb{R})}<\infty
\end{array}
\end{array} .\right.
$$


Obviously $E^{\star} \leq E_{F S}^{\star}$. Kenig and Merle [2008] chose this definition. The advantage of using $E^{\star}$ is that $E^{\star} \geq m$ implies uniform bound on the global Strichartz norms below $m$, which is very important in applications where we want to perturb the equation.

The next lemma is the conclusion of this section.

Lemma 6.1. Assume that $f$ satisfies (1-36), and let $u_{n}$ be a sequence of solutions of NLKG in $\mathscr{K}^{+}$on $I_{n} \subset \mathbb{R}$ satisfying

$$
E\left(u_{n}\right) \rightarrow E^{\star}<m, \quad\left\|u_{n}\right\|_{S T\left(I_{n}\right)} \rightarrow \infty \quad(n \rightarrow \infty) .
$$

Then there exists a global solution $u_{*}$ of $N L K G$ in $\mathscr{K}^{+}$satisfying

$$
E\left(u_{*}\right)=E^{\star}, \quad\left\|u_{*}\right\|_{S T(\mathbb{R})}=\infty .
$$

In addition, there are a sequence $\left(t_{n}, x_{n}\right) \in \mathbb{R} \times \mathbb{R}^{d}$ and $\varphi \in L^{2}\left(\mathbb{R}^{d}\right)$ such that along some subsequence,

$$
\left\|\vec{u}_{n}(0, x)-e^{-i\langle\nabla\rangle t_{n}} \varphi\left(x-x_{n}\right)\right\|_{L_{x}^{2}} \rightarrow 0 .
$$

We call such a global solution $u_{*}$ a critical element. Observe that by the definition of $E^{\star}$, we can find such a sequence $u_{n}$, once we have $E^{\star}<m$.

Proof. We can translate $u_{n}$ in $t$ so that $0 \in I_{n}$ for all $n$. Then we consider the linear and nonlinear profile decompositions of $u_{n}$, using Lemma 5.1 in the $H^{1}$ critical case (1-28) and Corollary 5.2 in the subcritical and exponential cases.

$$
\begin{aligned}
& e^{i\langle\nabla\rangle t} \vec{u}_{n}(0)=\sum_{j<k} \vec{v}_{n}^{j}+\vec{w}_{n}^{k}, \quad \vec{v}_{n}^{j}=e^{i\langle\nabla\rangle\left(t-t_{n}^{j}\right)} T_{n}^{j} \varphi^{j}, \\
& u_{(n)}^{<k}=\sum_{j<k} u_{(n)}^{j}, \quad \vec{u}_{(n)}^{j}=T_{n}^{j} \vec{U}_{\infty}^{j}\left(\left(t-t_{n}^{j}\right) / h_{n}^{j}\right), \\
& \left\|\vec{v}_{n}^{j}(0)-\vec{u}_{(n)}^{j}(0)\right\|_{L_{x}^{2}} \rightarrow 0 \quad(n \rightarrow \infty) .
\end{aligned}
$$

Lemma 5.6 precludes that all the nonlinear profiles $\vec{U}_{\infty}^{j}$ have finite global Strichartz norm. On the other hand, every solution of NLKG in $\mathscr{K}^{+}$with energy less than $E^{\star}$ has global finite Strichartz norm by the definition of $E^{\star}$. Hence by Lemma 5.3 we deduce that there is only one profile i.e. $K=1$, and moreover

$$
\tilde{E}\left(\vec{u}_{(n)}^{0}\right)=E^{\star}, \quad \vec{u}_{(n)}^{0}(0) \in \widetilde{\mathscr{K}}^{+}, \quad\left\|\widehat{U}_{\infty}^{0}\right\|_{S T_{\infty}^{0}(\mathbb{R})}=\infty, \quad \lim _{n \rightarrow \infty}\left\|\vec{w}_{n}^{1}\right\|_{L_{t}^{\infty} L_{x}^{2}}=0 .
$$

If $h_{n}^{0} \rightarrow 0$ in the critical case, then $\hat{U}_{\infty}^{0}=|\nabla|^{-1} \operatorname{Re} \vec{U}_{\infty}^{0}$ solves the massless equation

$$
\left(\partial_{t}^{2}-\Delta\right) \hat{U}_{\infty}^{0}=f^{\prime}\left(\hat{U}_{\infty}^{0}\right)
$$

and satisfies

$$
E^{0}\left(\hat{U}_{\infty}^{0}\right)=E^{\star}<m=J^{(0)}(Q), \quad K^{w}\left(\hat{U}_{\infty}^{0}(0)\right) \geq 0, \quad\left\|\hat{U}_{\infty}^{0}\right\|_{[W]_{2}^{\bullet}}=\infty,
$$

where $Q$ is the massless ground state and $K^{w}$ is the massless version of $K$. However, there is no such solution, by [Kenig and Merle 2008]. ${ }^{5}$ Hence $h_{n}^{0} \equiv 1$ in all cases, and we obtain (6-5).

\footnotetext{
${ }^{5}$ That reference is restricted to the dimensions $d \leq 5$ for simplicity of the perturbation argument, but the elimination of critical elements works in any higher dimensions.
} 
Hence $h_{n}^{0} \equiv 1$ in all cases, and we obtain (6-5).

It remains to prove that $\widehat{U}_{\infty}^{0}=\langle\nabla\rangle^{-1} \operatorname{Re} \vec{U}_{\infty}^{0}$ is a global solution. Suppose not. Then we can choose a sequence $t_{n} \in \mathbb{R}$ approaching the maximal existence time. Since the sequence of solutions $\hat{U}_{\infty}^{0}\left(t+t_{n}\right)$ satisfies the assumption of this lemma, we may apply the above argument to it. In particular, from (6-5) we obtain

$$
\left\|\vec{U}_{\infty}^{0}\left(t_{n}\right)-e^{-i\langle\nabla\rangle t_{n}^{\prime}} \psi\left(x-x_{n}^{\prime}\right)\right\|_{L_{x}^{2}} \rightarrow 0
$$

for some $\psi \in L_{x}^{2}$ and another sequence $\left(t_{n}^{\prime}, x_{n}^{\prime}\right) \in \mathbb{R} \times \mathbb{R}^{d}$. Let $\vec{v}:=e^{i\langle\nabla\rangle t} \psi$. Since it is a free solution, for any $\varepsilon>0$ there is $\delta>0$ such that for any interval $I$ satisfying $|I| \leq 2 \delta$, we have $\left\|\langle\nabla\rangle^{-1} \vec{v}\right\|_{S T(I)} \leq \varepsilon$, where $S T=[W]_{2} \cap[K]_{2}$ as in (5-59). Then (6-10) implies that

$$
\varlimsup_{n \rightarrow \infty}\left\|\langle\nabla\rangle^{-1} e^{i\langle\nabla\rangle t} \vec{U}_{\infty}^{0}\left(t_{n}\right)\right\|_{S T(-\delta, \delta)} \leq \varepsilon .
$$

If $\varepsilon>0$ is small enough, this implies that the solution $\hat{U}_{\infty}^{0}$ exists on $\left(t_{n}-\delta, t_{n}+\delta\right)$, by the iteration argument, for large $n$. This contradicts the choice of $t_{n}$. Hence $\widehat{U}_{\infty}^{0}$ is global and so a critical element.

\section{Extinction of the critical element}

In this section, we prove that the critical element can not exist by deriving a contradiction from a few properties of it. The main idea follows [Kenig and Merle 2006; 2008]. Let $u_{c}$ be a critical element given by Lemma 6.1. Since NLKG is symmetric in $t$, we may assume that $\left\|u_{c}\right\|_{S T(0, \infty)}=\infty$. We call such $u$ a forward critical element. Note that since the critical element is in $\mathscr{K}^{+}$, we have $E_{(u ; t)}^{Q_{(}} \sim E(u)$ uniformly, by Lemma 2.10 .

Compactness. First we show that the trajectory of a forward critical element is precompact for positive time in the energy space modulo spatial translations.

Lemma 7.1. Assume that $f$ satisfies (1-36), and let $u_{c}$ be a forward critical element. Then there exists $c:(0, \infty) \rightarrow \mathbb{R}^{d}$ such that the set

$$
\{(u, \dot{u})(t, x-c(t)) \mid 0<t<\infty\}
$$

is precompact in $H^{1}\left(\mathbb{R}^{d}\right) \times L^{2}\left(\mathbb{R}^{d}\right)$.

Proof. The proof in [Kenig and Merle 2008] can be adapted verbatim, but we give a sketch for the sake of completeness. Recall the convention $u \leftrightarrow \vec{u}$ defined on page 427 .

It suffices to prove precompactness of $\left\{\vec{u}\left(t_{n}\right)\right\}$ in $L_{x}^{2}$ for any $t_{1}, t_{2}, \cdots>0$. If $t_{n}$ converges, then it is trivial from the continuity in $t$. Hence we may assume that $t_{n} \rightarrow \infty$. Applying Lemma 6.1 to the sequence of solutions $u\left(t+t_{n}\right)$, we get another sequence $\left(t_{n}^{\prime}, x_{n}^{\prime}\right) \in \mathbb{R}^{1+d}$ and $\varphi \in L^{2}$ such that

$$
\vec{u}\left(t_{n}, x\right)-e^{-i\langle\nabla\rangle t_{n}^{\prime}} \varphi\left(x-x_{n}^{\prime}\right) \rightarrow 0 \text { in } L_{x}^{2} \quad(n \rightarrow \infty) .
$$

If $t_{n}^{\prime} \rightarrow-\infty$, then we have

$$
\left\|e^{i\langle\nabla\rangle t} \vec{u}\left(t_{n}\right)\right\|_{S T(0, \infty)}=\left\|e^{i\langle\nabla\rangle t} \varphi\right\|_{S T\left(-t_{n}^{\prime}, \infty\right)}+o(1) \rightarrow 0,
$$

so that we can solve NLKG of $u$ for $t>t_{n}$ with large $n$ globally by iteration with small Strichartz norms, contradicting its forward criticality. 
If $t_{n}^{\prime} \rightarrow+\infty$, then we have

$$
\left\|e^{i\langle\nabla\rangle t} \vec{u}\left(t_{n}\right)\right\|_{S T(-\infty, 0)}=\left\|e^{i\langle\nabla\rangle t} \varphi\right\|_{S T\left(-\infty,-t_{n}^{\prime}\right)}+o(1) \rightarrow 0,
$$

so that we can solve NLKG of $u$ for $t<t_{n}$ with large $n$ with diminishing Strichartz norms, which implies $u=0$ by taking the limit, a contradiction.

Thus $t_{n}^{\prime}$ is precompact, so is $\vec{u}\left(t_{n}, x+x_{n}^{\prime}\right)$ in $L_{x}^{2}$ by (7-2).

As a consequence, the energy of $u$ stays within a fixed radius for all positive time, modulo arbitrarily small rest. More precisely, we define the exterior energy by

$$
E_{R, c}(u ; t)=\int_{|x-c| \geq R}\left|u_{t}\right|^{2}+|\nabla u|^{2}+|u|^{2}+|f(u)|+\left|u f^{\prime}(u)\right| d x,
$$

for any $R>0$ and $c \in \mathbb{R}^{d}$. Then we have

Corollary 7.2. Let $u$ be a forward critical element. Then for any $\varepsilon>0$, there exist $R_{0}(\varepsilon)>0$ and $c(t):(0, \infty) \rightarrow \mathbb{R}^{d}$ such that at any $t>0$ we have

$$
E_{R_{0}, c(t)}(u ; t) \leq \varepsilon E(u) .
$$

Zero momentum and non-propagation. Next we observe that the critical element can not move with any positive speed in the sense of energy. For that we first need to see that the (conserved) momentum

$$
P(u):=\int_{\mathbb{R}^{d}} u_{t} \nabla u d x \in \mathbb{R}^{d}
$$

is zero for any critical element $u$.

Lemma 7.3. For any critical element $u$, we have $P(u)=0$.

Proof. For $j=1, \ldots, d$ and $\lambda \in \mathbb{R}$, we define the operator $L_{j}^{\lambda}$ of Lorentz transformation:

$$
\begin{gathered}
L_{j}^{\lambda} u\left(x_{0}, \ldots, x_{d}\right)=u\left(y_{0}, \ldots, y_{d}\right), \\
y_{0}=x_{0} \cosh \lambda+x_{j} \sinh \lambda, \quad y_{j}=x_{0} \sinh \lambda+x_{j} \cosh \lambda, \quad y_{k}=x_{k}(k \neq 0, j) .
\end{gathered}
$$

Then $L_{j}^{\alpha} L_{j}^{\beta}=L_{j}^{\alpha+\beta}$. Since $\partial_{\lambda} y_{0}=y_{j}$ and $\partial_{\lambda} y_{j}=y_{0}$, we have

$$
\partial_{\lambda} L_{j}^{\lambda} u=L_{j}^{\lambda}\left[\left(x_{j} \partial_{t}+t \partial_{j}\right) u\right]
$$

Also we have

$$
\begin{array}{ll}
\partial_{t} L_{j}^{\lambda}=L_{j}^{\lambda}\left(s \partial_{t}+c \partial_{j}\right), & \partial_{t t} L_{j}^{\lambda}=L_{j}^{\lambda}\left(s^{2} \partial_{t t}+2 s c \partial_{t j}+c^{2} \partial_{j j}\right), \\
\partial_{j} L_{j}^{\lambda}=L_{j}^{\lambda}\left(c \partial_{t}+s \partial_{j}\right), & \partial_{j j} L_{j}^{\lambda}=L_{j}^{\lambda}\left(c^{2} \partial_{t t}+2 s c \partial_{t j}+s^{2} \partial_{j j}\right),
\end{array}
$$

where $s:=\sinh \lambda$ and $c:=\cosh \lambda$. In particular $\left[\partial_{t}^{2}-\Delta, L_{j}^{\lambda}\right]=0$, and so $L_{j}^{\lambda}$ maps global solutions to themselves. For the space-time norm, we have

$$
\iint L_{j}^{\lambda} v d t d x_{j}=\iint v\left|\left(\begin{array}{ll}
c & s \\
s & c
\end{array}\right)\right| d t d x_{j}=\iint v d t d x_{j}
$$


hence $L_{j}^{\lambda}$ preserves all $L_{t, x}^{p}\left(\mathbb{R}^{1+d}\right)$ norm. For any solution $u$, we have

$$
\begin{aligned}
\partial_{\lambda}^{0} E\left(L_{j}^{\lambda} u\right) & =\left\langle u_{t} \mid \partial_{\lambda}^{0} \partial_{t} L_{j}^{\lambda} u\right\rangle+\left\langle\nabla u \mid \partial_{\lambda}^{0} \nabla L_{j}^{\lambda} u\right\rangle+\left\langle u-f^{\prime}(u) \mid \partial_{\lambda}^{0} L_{j}^{\lambda} u\right\rangle \\
& =\left\langle u_{t} \mid x_{j} u_{t t}+t u_{t j}+u_{j}\right\rangle+\left\langle u_{k} \mid x_{j} u_{k t}+t u_{k j}+\delta_{k j} u_{t}\right\rangle+\left\langle u-f^{\prime}(u) \mid x_{j} u_{t}+t u_{j}\right\rangle \\
& =\left\langle x_{j} u_{t} \mid \Delta u\right\rangle+2\left\langle u_{t} \mid u_{j}\right\rangle-\left\langle x_{j} u_{k t} \mid u_{k}\right\rangle=\left\langle u_{t} \mid u_{j}\right\rangle=P(u),
\end{aligned}
$$

where $\partial_{\lambda}^{0}:=\left.\partial_{\lambda}\right|_{\lambda=0}$. If $P_{j}(u) \neq 0$ for some $j$, then we obtain another global solution $L_{j}^{\lambda} u$, which has smaller energy and infinite Strichartz norm. It also belongs to $\mathscr{K}^{+}$, by continuity. More precisely, the continuity of $L_{j}^{\lambda} u$ in $\lambda$ in the energy space easily follows from the local wellposedness if $u$ has compactly supported initial data. Then the original solution is approximated by smooth cut-off using the finite propagation property. Thus we obtain another critical element with less energy, a contradiction. Hence $P(u)=0$.

Next we see stillness of critical elements in terms of the energy propagation. For any $R>0$, we define the localized center of energy $X_{R}(t) \in \mathbb{R}^{d}$ by

$$
X_{R}(u ; t):=\int \chi_{R}(x) x e(u)(t, x) d x,
$$

where $\chi_{R}$ is as defined in (1-23), and $e(u)$ denote the energy density of $u$, namely

$$
e(u)=\left(\left|u_{t}\right|^{2}+|\nabla u|^{2}+|u|^{2}\right) / 2-f(u) .
$$

From the energy identity $\dot{e}(u)=\nabla \cdot\left(u_{t} \nabla u\right)$, we get for any solution $u$

$$
\frac{d}{d t} X_{R}(u ; t)=-d P(u)+\int\left[d\left(1-\chi_{R}(x)\right)+\left(r \partial_{r}\right) \chi_{R}(x)\right] u_{t} \nabla u
$$

If $u$ is a critical element, the first term disappears by the above lemma, so we have

$$
\left|\frac{d}{d t} X_{R}(u ; t)\right| \lesssim E_{R, 0}(u ; t)
$$

Moreover, since $u$ is in $\mathscr{K}^{+}$, by Lemma 2.12 there exists $\delta_{0} \in(0,1)$ such that

$$
K_{1,0}(u(t)) \geq \delta_{0}\|u(t)\|_{H^{1}}^{2} \quad \text { for all } t \in \mathbb{R} .
$$

Lemma 7.4. Let $u$ be a forward critical element, and let $R_{0}(\varepsilon)>0, c(t) \in \mathbb{R}^{d}$ and $\delta_{0}>0$ be as in (7-6) and (7-17). If $0<\varepsilon \ll \delta_{0}$ and $R \gg R_{0}(\varepsilon)$ then we have

$$
|c(t)-c(0)| \leq R-R_{0}(\varepsilon),
$$

for $0<t<t_{0}$ till some $t_{0} \gtrsim \delta_{0} R / \varepsilon$.

Proof. By translation in $x$, we may assume that $c(0)=0$. Let $t_{0}$ be the final time for the above property

$$
t_{0}=\inf \left\{t>0|| c(t) \mid \geq R-R_{0}\right\} .
$$


Then the finite speed of propagation implies that $t_{0}>0$. For any $0<t<t_{0}$ we have $|c(t)| \leq R-R_{0}$, hence by (7-6) we have $E_{R, 0} \leq \varepsilon E(u)$, and so by (7-16) we get

$$
\left|\frac{d}{d t} X_{R}(u ; t)\right| \lesssim \varepsilon E(u) .
$$

Next we expand it around $c$ :

$$
c(t) \cdot X_{R}(u ; t)=|c(t)|^{2} \int \chi_{R}(x) e(u) d x+\int \chi_{R}(x) c \cdot(x-c) e(u) d x,
$$

where the first term on the right is bounded from below by

$$
\begin{aligned}
E(u)-\int\left(1-\chi_{R}(x)\right) e(u) d x & \geq\|\dot{u}(t)\|_{L_{x}^{2}}^{2} / 2+K_{1,0}(u(t))-C E_{R, 0}(t) \\
& \geq \delta_{0} E(u)-C \varepsilon E(u) \gtrsim \delta_{0} E(u),
\end{aligned}
$$

since $\varepsilon \ll \delta_{0}$. The second term of (7-21) is dominated by splitting the integral into $|x-c| \leq R_{0}$ and $|x-c| \geq R_{0}$. In the interior it is bounded by using the energy bound, and in the exterior it is bounded by using (7-6). Thus we obtain

$$
\left|\int \chi_{R}(x) c \cdot(x-c) e(u) d x\right| \lesssim\left(R_{0}+R \varepsilon\right) E(u)|c| .
$$

In the same way we have $\left|X_{R}(u ; 0)\right| \lesssim\left(R_{0}+R \varepsilon\right) E(u)$, since $c(0)=0$. Thus we get

$$
\delta_{0} E(u)|c(t)| \lesssim\left(R_{0}+R \varepsilon+\varepsilon t\right) E(u),
$$

and sending $t \rightarrow t_{0}$, we get $\delta_{0} R \lesssim \varepsilon t_{0}$.

Dispersion and contradiction. Finally we use the localized virial identity to see dispersion of the critical element, which will contradict the above non-propagation property. For any $R>0$, we define the localized virial $V_{R}(u ; t) \in \mathbb{R}$ by

$$
V_{R}(u ; t):=\left\langle\chi_{R}(x) u_{t} \mid(x \cdot \nabla+\nabla \cdot x) u\right\rangle,
$$

where $\chi_{R}$ is as defined in (1-23). Then we have for any solution $u$,

$$
\begin{aligned}
\frac{d}{d t} V_{R}(u ; t)= & -\int \chi_{R}(x)\left[2|\nabla u|^{2}-d(D-2) f(u)\right]+\frac{d}{2}|u|^{2} \Delta \chi_{R}(x) d x \\
& \quad-\int r \partial_{r} \chi_{R}(x)\left[\left|u_{t}\right|^{2}+2\left|u_{r}\right|^{2}-|\nabla u|^{2}-|u|^{2}+2 f(u)\right] d x \\
\leq- & K_{d,-2}(u(t))+C E_{R, 0}(u ; t) .
\end{aligned}
$$

If $u$ is a critical element, then $u \in \mathscr{K}^{+}$and hence by Lemma 2.12, there exists $\delta_{2} \in(0,1)$ such that

$$
K_{d,-2}(u(t)) \geq \delta_{2}\|\nabla u(t)\|_{L_{x}^{2}}^{2}
$$

for all $t>0$. Thus we obtain, integrating in $t$,

$$
V_{R}\left(u ; t_{0}\right) \leq V_{R}(u ; 0)-\delta_{2} \int_{0}^{t_{0}}\|\nabla u(t)\|_{L_{x}^{2}}^{2} d t+C \varepsilon E(u) t_{0} .
$$


Now by the compactness Lemma 7.1, we have:

Lemma 7.5. Let $u$ be a forward critical element. Then for any $\varepsilon>0$ there exists $C>0$ such that

$$
\|u(t)\|_{L_{x}^{2}}^{2} \leq C\|\nabla u(t)\|_{L_{x}^{2}}^{2}+\varepsilon\|\dot{u}(t)\|_{L_{x}^{2}}^{2}
$$

for all $t>0$.

Proof. Otherwise there exists a sequence $t_{n}>0$ such that

$$
\left\|u\left(t_{n}\right)\right\|_{L_{x}^{2}}^{2}>n\left\|\nabla u\left(t_{n}\right)\right\|_{L_{x}^{2}}^{2}+\varepsilon\left\|\dot{u}\left(t_{n}\right)\right\|_{L_{x}^{2}}^{2} .
$$

Since $u$ is $L_{x}^{2}$ bounded, it follows that $\left\|\nabla u\left(t_{n}\right)\right\|_{L_{x}^{2}} \rightarrow 0$. Then Lemma 7.1 implies that, after passing to a subsequence, $u\left(t_{n}\right) \rightarrow 0$ strongly in $H_{x}^{1}$, then the above inequality implies that $\dot{u}\left(t_{n}\right) \rightarrow 0$ too. Hence $E^{Q}\left(u ; t_{n}\right) \rightarrow 0$, which contradicts the energy equivalence, Lemma 2.10 .

Multiplying the equation with $u$, and then applying the above lemma with $\varepsilon=\frac{1}{4}$, we obtain

$$
\partial_{t}\langle u \mid \dot{u}\rangle=\int_{\mathbb{R}^{d}}|\dot{u}|^{2}-|\nabla u|^{2}-|u|^{2}+D f(u) d x \geq \int_{\mathbb{R}^{d}}|\dot{u}|^{2} / 2+|u|^{2}-C|\nabla u|^{2} d x,
$$

with some $C>0$. Hence

$$
\int_{0}^{t_{0}}\|\dot{u}\|_{L_{x}^{2}}^{2}+\|u\|_{L_{x}^{2}}^{2} d t \lesssim E(u)+\int_{0}^{t_{0}}\|\nabla u\|_{L_{x}^{2}}^{2} d t
$$

and so

$$
t_{0} E(u) \leq \int_{0}^{t_{0}} E^{Q}(u ; t) d t \lesssim E(u)+\int_{0}^{t_{0}}\|\nabla u\|_{L_{x}^{2}}^{2} d t
$$

Now we choose positive $\varepsilon \ll \delta_{2} \delta_{0}$ and $R \gg R_{0}(\varepsilon)$. Then by Lemma 7.4 there exists $t_{0} \sim \delta_{0} R / \varepsilon$ such that $E_{R, 0}(u ; t) \leq \varepsilon E(u)$ for $0<t<t_{0}$. Then from (7-28) and (7-33), we have

$$
-V_{R}\left(u ; t_{0}\right)+V_{R}(u ; 0) \gtrsim\left[\delta_{2} t_{0}-C \varepsilon t_{0}-C\right] E(u) \gtrsim \delta_{2} t_{0} E(u) \sim \frac{\delta_{2} \delta_{0} R}{\varepsilon} E(u),
$$

while the left-hand side is dominated by $R E(u)-$ a contradiction when $\varepsilon / \delta_{2} \delta_{0}$ is small enough.

\section{Appendix: The range of scaling exponents}

In Section 2, we have shown that $m_{\alpha, \beta}$ in (1-17) is positive and achieved (after modification of the mass in the critical and exponential cases) if $(\alpha, \beta)$ satisfies (1-16). Here we see that it is also necessary, modulo the obvious symmetry $(\alpha, \beta) \rightarrow(-\alpha,-\beta)$. For simplicity, we consider only the pure power nonlinearity.

Proposition A.1. Assume that neither $(\alpha, \beta) \in \mathbb{R}^{2}$ nor $(-\alpha,-\beta)$ satisfies (1-16). There exists $q \in$ $\left(2_{\star}, 2^{\star}\right)$ such that $m_{\alpha, \beta}=-\infty$ for $f(\varphi)=|\varphi|^{q}$.

Proof. By symmetry with respect to $(\alpha, \beta) \rightarrow(-\alpha,-\beta)$, we may assume that $\beta>0$ and $\bar{\mu}=2 \alpha+d \beta>0$.

First we consider the case $\alpha<0$ and $\underline{\mu}>0$, which implies that $d \geq 2$. Let $\left(2_{\star}, 2^{\star}\right) \ni q=2+p$, then we have

$$
\alpha p+\bar{\mu} \geq d \underline{\mu} /(d-2)>0
$$


Decompose $K(\varphi)$ by setting

$$
K=K_{1}+K_{2}, \quad K_{1}(\varphi)=\underline{\mu} \frac{\|\nabla \varphi\|_{L^{2}}^{2}}{2}, \quad K_{2}(\varphi)=\bar{\mu} \frac{\|\varphi\|_{L^{2}}^{2}}{2}-(\alpha p+\bar{\mu}) F(\varphi) .
$$

Suppose that $0 \neq \varphi \in H^{1}\left(\mathbb{R}^{d}\right)$ satisfies $K_{2}(\varphi)=0$. If there is no such $\varphi$, then $K$ is positive definite and the minimization set in (1-17) becomes empty. Let $1<v \rightarrow 1+0$. Then

$$
0>K_{2}(v \varphi) \rightarrow K_{2}(\varphi)=0, \quad K_{1}(v \varphi) \rightarrow K_{1}(\varphi)>0 .
$$

Now let $\lambda(v)>0$ solve

$$
0=K(\nu \varphi(x / \lambda))=\lambda^{d-2} K_{1}(\nu \varphi)+\lambda^{d} K_{2}(v \varphi)
$$

in other words $\lambda(v)=\left[-K_{2}(v \varphi) / K_{1}(v \varphi)\right]^{1 / 2}$. Then $\lambda(v) \rightarrow \infty$ as $v \rightarrow 1+0$ due to (A-3). Since

$$
\bar{\mu} J(\psi)=K(\psi)+\beta\|\nabla \psi\|_{L^{2}}^{2}+\alpha p F(\psi),
$$

we obtain

$$
\bar{\mu} J(v \varphi(x / \lambda))=\beta v^{2} \lambda^{d-2}\|\nabla \varphi\|_{L^{2}}^{2}+\alpha p \lambda^{d} F(v \varphi) \rightarrow-\infty,
$$

which implies that $m=-\infty$.

Next, if $\bar{\mu}=0>\alpha$, which implies $d \geq 2$, then for any nonzero $\varphi \in H^{1}\left(\mathbb{R}^{d}\right)$ satisfying $K(\varphi)=0$ we have

$$
K(\varphi(x / \lambda))=\lambda^{d} K(\varphi)=0
$$

and similarly as above,

$$
J(\varphi(x / \lambda))=O\left(-\lambda^{d}\right) \rightarrow \infty \quad \text { as } \lambda \rightarrow \infty .
$$

Finally consider the case $\underline{\mu}<0<\bar{\mu}$. Then $\alpha p+2 \beta=0$ has a solution $p \in\left(4 / d, 2^{\star}-2\right)$. Since $\alpha p+\bar{\mu}=\alpha p+2 \beta+\underline{\mu}$, there exists $p \in\left(4 / d, 2^{\star}-2\right)$ such that

$$
\alpha p+\bar{\mu}<0<\alpha p+2 \beta .
$$

Then $K^{N}(\varphi)=-(\alpha p+\bar{\mu}) F(\varphi)$ is positive and so for any $\varphi \in H^{1}\left(\mathbb{R}^{d}\right), K(v \varphi) \geq 0$ if $v \gg 1$. Since the kinetic term in $K$ is negative, there exists $\xi(v) \in \mathbb{R}^{d}$ such that $K\left(e^{i \xi x} v \varphi\right)=0$. Since

$$
-\underline{\mu} J(\psi)=-K(\psi)+2 \beta \frac{\|\varphi\|_{L^{2}}^{2}}{2}-(\alpha p+2 \beta) F(\psi)
$$

we obtain

$$
-\underline{\mu} J\left(e^{i \xi x} \nu \varphi\right)=2 \beta v^{2} \frac{\|\varphi\|_{L^{2}}^{2}}{2}-(\alpha p+2 \beta) F(\nu \psi) \rightarrow-\infty,
$$

which implies that $m=-\infty$.

The above proof shows that if $\alpha<0$ and $\underline{\mu} \geq 0$ then $m=-\infty$ for all $q \in\left(2,2^{\star}\right]$. The choice of $q$ was needed only in the other region.

\section{Acknowledgments}

The authors thank Guixiang Xu for pointing out several mistakes in the first manuscript. S. Ibrahim is partially supported by NSERC\# 371637-2009 grant and a start up fund from University of Victoria. 


\section{Table of Notation}

Notation applies to any $s \in \mathbb{R}, v \geq 0,(\alpha, \beta) \in \mathbb{R}^{2}, j, k \in \mathbb{Z}, Z \in \mathbb{R}^{3}, I \subset \mathbb{R}, \varphi, \psi \in H^{1}\left(\mathbb{R}^{d}\right)$, $u \in C_{t}\left(H_{x}^{1}\left(\mathbb{R}^{d}\right)\right)$, any suffix $\diamond, \bigcirc$, any sequence $\varphi_{n} \in H^{1}\left(\mathbb{R}^{d}\right)$, and any functional $G$ on $H^{1}\left(\mathbb{R}^{d}\right)$.

\begin{tabular}{|c|c|c|}
\hline Dimension and scaling & $\begin{array}{l}d \in \mathbb{N}, 2_{\star}, 2^{\star}>0 \text { : space dimension and critical powers } \\
\alpha, \beta \in \mathbb{R}, \bar{\mu} \geq \underline{\mu} \geq 0 \text { : scaling exponents and their functions } \\
\varphi_{\alpha, \beta}^{\lambda}, \mathscr{L}_{\alpha, \beta} G: \text { rescaled family and scaling derivative } \\
\quad\left(\text { subscript } \diamond_{\alpha, \beta} \text { and } \bigcirc_{\alpha, \beta} \text { are often written as } \diamond \text { and } \varnothing\right)\end{array}$ & $\begin{array}{r}(1-3) \\
(2-1) \\
(1-13)(1-14)\end{array}$ \\
\hline 1st order representation & $\vec{u} \leftrightarrow u$ : linked with each other by & $(4-1)$ \\
\hline Nonlinearity & $\begin{array}{l}F(\varphi), f(s) \geq 0 \text { : nonlinear energy and its density } \\
f_{S}(s), f_{L}(s) \geq 0 \text { : small and large parts of } f \\
p_{1}, p_{2}>0, \kappa_{0} \geq 0 \text { : leading powers of } f_{S} \text { and } f_{L}\end{array}$ & $\begin{array}{r}(1-11) \\
(1-24) \\
(1-26)(1-29)\end{array}$ \\
\hline Functionals & 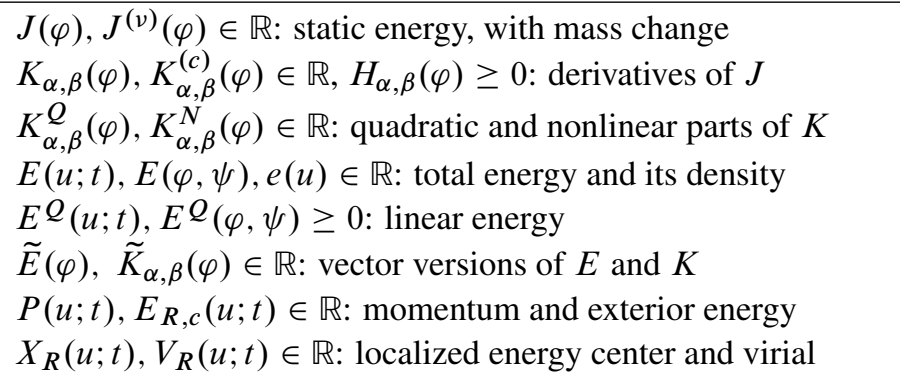 & $\begin{array}{r}(1-11)(1-12) \\
(1-15)(2-26) \\
(2-2) \\
(1-5)(7-14) \\
(1-37) \\
(4-3) \\
(7-7)(7-5) \\
(7-13)(7-25)\end{array}$ \\
\hline Variational splittings & $\begin{array}{l}m_{\alpha, \beta}, E^{\star} \geq 0: \text { static and scattering energy thresholds } \\
\mathscr{K}_{\alpha, \beta}^{ \pm}, \widetilde{\mathscr{K}}_{\alpha, \beta}^{+}: \text {splitting below the threshold } \\
C_{\mathrm{TM}}^{v}(G), C_{\mathrm{TM}}^{\star}(G) \in[0, \infty]: \text { Trudinger-Moser ratio } \\
\mathfrak{M}(G) \in[0, \infty]: \text { Trudinger-Moser threshold on } \dot{H}^{1} \\
\text { conc } G\left(\left(\varphi_{n}\right)_{n}\right) \in \mathbb{R}: \text { concentration at } x=0\end{array}$ & $\begin{array}{r}(1-17)(6-1) \\
(1-18)(4-5) \\
(2-47)(2-49) \\
(2-48) \\
(2-51) \\
\end{array}$ \\
\hline $\begin{array}{c}\text { Function spaces } \\
\text { and exponents }\end{array}$ & $\begin{array}{l}{[Z]_{v}(I),[Z]_{0}(I),[Z]_{v}^{\bullet}(I): \text { Lebesgue-Besov spaces on } I \times \mathbb{R}^{d}} \\
Z^{s}, Z^{*(s)} \in \mathbb{R}^{3}: \text { regularity change and dual of exponents } \\
\operatorname{reg}^{\theta}(Z), \operatorname{str}^{\theta}(Z), \operatorname{dec}^{\theta}(Z) \in \mathbb{R}: \text { regularity and decay indexes } \\
H, W, K, M^{\sharp}, V \in \mathbb{R}^{3}: \text { exponents for } d \in \mathbb{N} \\
X, S, L \in \mathbb{R}^{3}: \text { exponents for } d \leq 4 \\
\widetilde{M}, M, \hat{M}, \widetilde{N}, N, Q, P, Y, R, G \in \mathbb{R}^{3}: \text { exponents for } d \geq 5 \\
H_{\varepsilon}, W_{\varepsilon}, M_{\varepsilon}^{\sharp} \in \mathbb{R}^{3}: \text { exponents for } d \geq 5 \\
H_{v}^{1}, M \mathcal{M}: H^{1}\left(\mathbb{R}^{2}\right) \text { and a set of Fourier multipliers on } \mathbb{R}^{d} \\
\mathscr{X}, \mathscr{Y}, \mathscr{Y}_{0}, \widetilde{\mathscr{Y}}, \mathscr{Y}_{0}^{*}, \mathscr{Y}^{*}: \text { Strichartz-type spaces } \\
S T(I), S T^{*}(I), S T_{\infty}^{\diamond}(I): \text { Strichartz-type spaces on } I \times \mathbb{R}^{d}\end{array}$ & $\begin{array}{r}(4-7) \\
(4-9) \\
(4-8) \\
(4-31)(5-68) \\
(4-31)(4-54) \\
(4-69)(5-87) \\
(5-81) \\
(4-38)(5-2) \\
(4-54)(4-77) \\
(5-59)(5-60)\end{array}$ \\
\hline Profile decomposition & 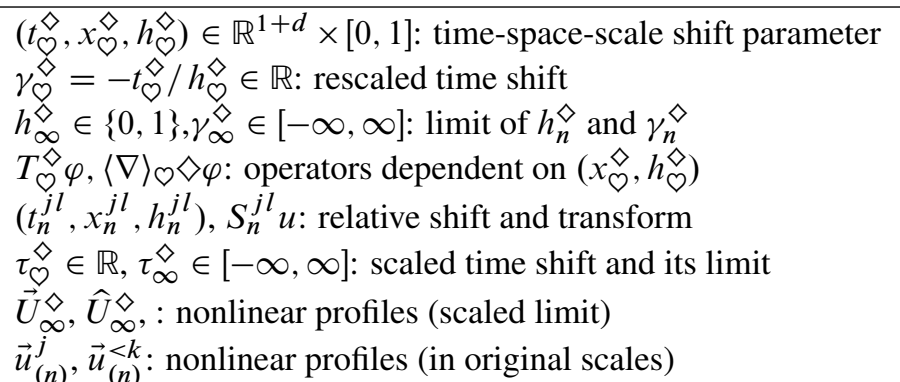 & $\begin{array}{r}\text { page } 438 \\
\\
(5-1) \\
(5-21)(5-20) \\
(5-1) \\
(5-52)(5-53) \\
(5-54)(5-58)\end{array}$ \\
\hline
\end{tabular}




\section{References}

[Adachi and Tanaka 2000] S. Adachi and K. Tanaka, "Trudinger type inequalities in $\mathbf{R}^{N}$ and their best exponents", Proc. Amer. Math. Soc. 128:7 (2000), 2051-2057. MR 2000m:46069 Zbl 0980.46020

[Adimurthi 1990] Adimurthi, "Existence of positive solutions of the semilinear Dirichlet problem with critical growth for the n-Laplacian”, Ann. Scuola Norm. Sup. Pisa Cl. Sci. (4) 17:3 (1990), 393-413. MR 91j:35016 Zbl 0732.35028

[Adimurthi and Struwe 2000] Adimurthi and M. Struwe, "Global compactness properties of semilinear elliptic equations with critical exponential growth", J. Funct. Anal. 175:1 (2000), 125-167. MR 2001g:35063 Zbl 0956.35045

[Akahori and Nawa 2010] T. Akahori and H. Nawa, "Blowup and scattering problems for the nonlinear Schrödinger equations", preprint, 2010. arXiv 1006.1485

[Bahouri and Gérard 1999] H. Bahouri and P. Gérard, "High frequency approximation of solutions to critical nonlinear wave equations", Amer. J. Math. 121:1 (1999), 131-175. MR 2000i:35123 Zbl 0919.35089

[Bahouri and Shatah 1998] H. Bahouri and J. Shatah, "Decay estimates for the critical semilinear wave equation", Ann. Inst. H. Poincaré Anal. Non Linéaire 15:6 (1998), 783-789. MR 99h:35136 Zbl 0924.35084

[Brenner 1984] P. Brenner, "On space-time means and everywhere defined scattering operators for nonlinear Klein-Gordon equations", Math. Z. 186:3 (1984), 383-391. MR 85h:35183 Zbl 0524.35084

[Carleson and Chang 1986] L. Carleson and S.-Y. A. Chang, "On the existence of an extremal function for an inequality of J. Moser”, Bull. Sci. Math. (2) 110:2 (1986), 113-127. MR 88f:46070

[Côte et al. 2008] R. Côte, C. E. Kenig, and F. Merle, "Scattering below critical energy for the radial 4D Yang-Mills equation and for the 2D corotational wave map system", Comm. Math. Phys. 284:1 (2008), 203-225. MR 2010k:35312 Zbl 1170.35064

[Duyckaerts et al. 2008] T. Duyckaerts, J. Holmer, and S. Roudenko, "Scattering for the non-radial 3D cubic nonlinear Schrödinger equation”, Math. Res. Lett. 15:6 (2008), 1233-1250. MR 2010e:35257

[Figueiredo et al. 1995] D. G. de Figueiredo, O. H. Miyagaki, and B. Ruf, "Elliptic equations in $\mathbf{R}^{2}$ with nonlinearities in the critical growth range", Calc. Var. Partial Differential Equations 3:2 (1995), 139-153. MR 97c:35063a Zbl 0820.35060

[Flucher 1992] M. Flucher, "Extremal functions for the Trudinger-Moser inequality in 2 dimensions", Comment. Math. Helv. 67:3 (1992), 471-497. MR 93k:58073 Zbl 0763.58008

[Ginibre and Velo 1985a] J. Ginibre and G. Velo, "The global Cauchy problem for the nonlinear Klein-Gordon equation", Math. Z. 189:4 (1985), 487-505. MR 86f:35149 Zbl 0549.35108

[Ginibre and Velo 1985b] J. Ginibre and G. Velo, "Time decay of finite energy solutions of the nonlinear Klein-Gordon and Schrödinger equations”, Ann. Inst. H. Poincaré Phys. Théor. 43:4 (1985), 399-442. MR 87g:35208

[Ibrahim et al. 2007] S. Ibrahim, M. Majdoub, and N. Masmoudi, "Double logarithmic inequality with a sharp constant", Proc. Amer. Math. Soc. 135:1 (2007), 87-97. MR 2008a:46034 Zbl 1130.46018

[Ibrahim et al. 2009] S. Ibrahim, M. Majdoub, N. Masmoudi, and K. Nakanishi, "Scattering for the two-dimensional energycritical wave equation", Duke Math. J. 150:2 (2009), 287-329. MR 2010k:35313 Zbl 1206.35175

[Ibrahim et al. 2011] S. Ibrahim, N. Masmoudi, and K. Nakanishi, "Trudinger-Moser inequality on the whole plane with the exact growth condition", preprint, 2011. arXiv 1110.1712

[Jeanjean and Le Coz 2009] L. Jeanjean and S. Le Coz, "Instability for standing waves of nonlinear Klein-Gordon equations via mountain-pass arguments", Trans. Amer. Math. Soc. 361:10 (2009), 5401-5416. MR 2010h:35269 Zbl 1176.35156

[Kenig and Merle 2006] C. E. Kenig and F. Merle, "Global well-posedness, scattering and blow-up for the energy-critical, focusing, non-linear Schrödinger equation in the radial case”, Invent. Math. 166:3 (2006), 645-675. MR 2007g:35232

[Kenig and Merle 2008] C. E. Kenig and F. Merle, "Global well-posedness, scattering and blow-up for the energy-critical focusing non-linear wave equation”, Acta Math. 201:2 (2008), 147-212. MR 2011a:35344 Zbl 1183.35202

[Killip et al. 2008] R. Killip, M. Visan, and X. Zhang, "The mass-critical nonlinear Schrödinger equation with radial data in dimensions three and higher", Anal. PDE 1:2 (2008), 229-266. MR 2011b:35487

[Killip et al. 2009] R. Killip, T. Tao, and M. Visan, "The cubic nonlinear Schrödinger equation in two dimensions with radial data”, J. Eur. Math. Soc. (JEMS) 11:6 (2009), 1203-1258. MR 2010m:35487

[Krieger and Schlag 2009] J. Krieger and W. Schlag, "Concentration compactness for critical wave maps", preprint, 2009. arXiv 0908.2474 
[Machihara et al. 2002] S. Machihara, K. Nakanishi, and T. Ozawa, "Nonrelativistic limit in the energy space for nonlinear Klein-Gordon equations", Math. Ann. 322:3 (2002), 603-621. MR 2003b:35199 Zbl 0991.35080

[Moser 1971] J. Moser, "A sharp form of an inequality by N. Trudinger", Indiana Univ. Math. J. 20 (1971), 1077-1092. MR 46 \#662 Zbl 0213.13001

[Nakanishi 1999a] K. Nakanishi, "Scattering theory for the nonlinear Klein-Gordon equation with Sobolev critical power", Internat. Math. Res. Notices 1 (1999), 31-60. MR 2000a:35174 Zbl 0933.35166

[Nakanishi 1999b] K. Nakanishi, "Energy scattering for nonlinear Klein-Gordon and Schrödinger equations in spatial dimensions 1 and 2", J. Funct. Anal. 169:1 (1999), 201-225. MR 2000m:35141

[Nakanishi 2001] K. Nakanishi, "Remarks on the energy scattering for nonlinear Klein-Gordon and Schrödinger equations", Tohoku Math. J. (2) 53:2 (2001), 285-303. MR 2002e:35220

[Ohta and Todorova 2007] M. Ohta and G. Todorova, "Strong instability of standing waves for the nonlinear Klein-Gordon equation and the Klein-Gordon-Zakharov system", SIAM J. Math. Anal. 38:6 (2007), 1912-1931. MR 2008a:35198 Zbl 1128.35074

[Payne and Sattinger 1975] L. E. Payne and D. H. Sattinger, "Saddle points and instability of nonlinear hyperbolic equations", Israel J. Math. 22:3-4 (1975), 273-303. MR 53 \#6112 Zbl 0317.35059

[Ruf 2005] B. Ruf, "A sharp Trudinger-Moser type inequality for unbounded domains in $\mathbb{R}^{2}$ ", J. Funct. Anal. 219:2 (2005), 340-367. MR 2005k:46082 Zbl 1119.46033

[Shatah 1985] J. Shatah, "Unstable ground state of nonlinear Klein-Gordon equations", Trans. Amer. Math. Soc. 290:2 (1985), 701-710. MR 86k:35088 Zbl 0617.35072

[Sterbenz and Tataru 2010] J. Sterbenz and D. Tataru, "Regularity of wave-maps in dimension $2+1$ ", Comm. Math. Phys. 298:1 (2010), 231-264. MR 2011h:58026 Zbl 1218.35057

[Tao 2008a] T. Tao, "Global regularity of wave maps, III: Large energy from $\mathbb{R}^{1+2}$ to hyperbolic spaces", preprint, 2008. arXiv 0805.4666

[Tao 2008b] T. Tao, "Global regularity of wave maps, IV: Absence of stationary or self-similar solutions in the energy class", preprint, 2008. arXiv 0806.3592

[Tao 2008c] T. Tao, "Global regularity of wave maps, V: Large data local wellposedness in the energy class", preprint, 2008. arXiv 0808.0368

[Tao 2009a] T. Tao, “Global regularity of wave maps, VI: Abstract theory of minimal-energy blowup solutions”, preprint, 2009. arXiv 0906.2833

[Tao 2009b] T. Tao, "Global regularity of wave maps, VII: Control of delocalised or dispersed solutions", preprint, 2009. arXiv 0908.0776

[Tao and Visan 2005] T. Tao and M. Visan, "Stability of energy-critical nonlinear Schrödinger equations in high dimensions", Electron. J. Differential Equations (2005), art. no. 118. MR 2006e:35307

[Tao et al. 2007] T. Tao, M. Visan, and X. Zhang, "The nonlinear Schrödinger equation with combined power-type nonlinearities", Comm. Partial Differential Equations 32:7-9 (2007), 1281-1343. MR 2009f:35324

[Zhang 2002] J. Zhang, "Sharp conditions of global existence for nonlinear Schrödinger and Klein-Gordon equations", Nonlinear Anal. A: Theory Methods 48:2 (2002), 191-207. MR 2002h:35303

Received 28 Jan 2010. Revised 11 May 2010. Accepted 8 Jun 2010.

SLIM IBRAHIM: ibrahim@math.uvic.ca

Department of Mathematics and Statistics, University of Victoria, PO Box 3060 STN CSC, Victoria V8P 5C3, Canada http://www.math.uvic.ca/ ibrahim/

NADER MASMOUDI: masmoudi@courant.nyu.edu

Courant Institute for Mathematical Sciences, New York University, New York, NY 10012-1185, United States

http://www.math.nyu.edu/faculty/masmoudi

KENJI NAKANISHI: n-kenji@math.kyoto-u.ac.jp

Department of Mathematics, Kyoto University, Kyoto 606-8502, Japan 


\section{Analysis \& PDE}

pjm.math.berkeley.edu/apde

EDITORS

EDITOR-IN-CHIEF

Maciej Zworski

University of California

Berkeley, USA

BOARD OF EDITORS

\begin{tabular}{|c|c|c|c|}
\hline Michael Aizenman & $\begin{array}{l}\text { Princeton University, USA } \\
\text { aizenman@math.princeton.edu }\end{array}$ & Nicolas Burq & $\begin{array}{l}\text { Université Paris-Sud 11, France } \\
\text { nicolas.burq@math.u-psud.fr }\end{array}$ \\
\hline Luis A. Caffarelli & $\begin{array}{l}\text { University of Texas, USA } \\
\text { caffarel@ math.utexas.edu }\end{array}$ & un-Yung Alice Chang & $\begin{array}{l}\text { Princeton University, USA } \\
\text { chang@math.princeton.edu }\end{array}$ \\
\hline Michael Christ & $\begin{array}{l}\text { University of California, Berkeley, USA } \\
\text { mchrist@math.berkeley.edu }\end{array}$ & Charles Fefferman & $\begin{array}{l}\text { Princeton University, USA } \\
\text { cf@math.princeton.edu }\end{array}$ \\
\hline Ursula Hamenstaedt & $\begin{array}{l}\text { Universität Bonn, Germany } \\
\text { ursula@math.uni-bonn.de }\end{array}$ & Nigel Higson & $\begin{array}{l}\text { Pennsylvania State Univesity, USA } \\
\text { higson@math.psu.edu }\end{array}$ \\
\hline Vaughan Jones & $\begin{array}{l}\text { University of California, Berkeley, USA } \\
\text { vfr@math.berkeley.edu }\end{array}$ & Herbert Koch & $\begin{array}{l}\text { Universität Bonn, Germany } \\
\text { koch@math.uni-bonn.de }\end{array}$ \\
\hline Izabella Laba & $\begin{array}{l}\text { University of British Columbia, Canada } \\
\text { ilaba@math.ubc.ca }\end{array}$ & Gilles Lebeau & $\begin{array}{l}\text { Université de Nice Sophia Antipolis, France } \\
\text { lebeau@unice.fr }\end{array}$ \\
\hline László Lempert & $\begin{array}{l}\text { Purdue University, USA } \\
\text { lempert@math.purdue.edu }\end{array}$ & Richard B. Melrose & $\begin{array}{l}\text { Massachussets Institute of Technology, USA } \\
\text { rbm@math.mit.edu }\end{array}$ \\
\hline Frank Merle & $\begin{array}{l}\text { Université de Cergy-Pontoise, France } \\
\text { Frank.Merle@u-cergy.fr }\end{array}$ & William Minicozzi II & $\begin{array}{l}\text { Johns Hopkins University, USA } \\
\text { minicozz@ math.jhu.edu }\end{array}$ \\
\hline Werner Müller & $\begin{array}{l}\text { Universität Bonn, Germany } \\
\text { mueller@math.uni-bonn.de }\end{array}$ & Yuval Peres & $\begin{array}{l}\text { University of California, Berkeley, USA } \\
\text { peres@stat.berkeley.edu }\end{array}$ \\
\hline Gilles Pisier & $\begin{array}{l}\text { Texas A\&M University, and Paris } 6 \\
\text { pisier@math.tamu.edu }\end{array}$ & Tristan Rivière & $\begin{array}{l}\text { ETH, Switzerland } \\
\text { riviere@ math.ethz.ch }\end{array}$ \\
\hline Igor Rodnianski & $\begin{array}{l}\text { Princeton University, USA } \\
\text { irod@math.princeton.edu }\end{array}$ & Wilhelm Schlag & $\begin{array}{l}\text { University of Chicago, USA } \\
\text { schlag@math.uchicago.edu }\end{array}$ \\
\hline Sylvia Serfaty & $\begin{array}{l}\text { New York University, USA } \\
\text { serfaty@ cims.nyu.edu }\end{array}$ & Yum-Tong Siu & $\begin{array}{l}\text { Harvard University, USA } \\
\text { siu@math.harvard.edu }\end{array}$ \\
\hline Terence Tao & $\begin{array}{l}\text { University of California, Los Angeles, USA } \\
\text { tao@math.ucla.edu }\end{array}$ & Michael E. Taylor & $\begin{array}{l}\text { Univ. of North Carolina, Chapel Hill, USA } \\
\text { met@math.unc.edu }\end{array}$ \\
\hline Gunther Uhlmann & $\begin{array}{l}\text { University of Washington, USA } \\
\text { gunther@math.washington.edu }\end{array}$ & András Vasy & $\begin{array}{l}\text { Stanford University, USA } \\
\text { andras@math.stanford.edu }\end{array}$ \\
\hline an Virgil Voiculescu & $\begin{array}{l}\text { University of California, Berkeley, USA } \\
\text { dvv@ math.berkeley.edu }\end{array}$ & Steven Zelditch & $\begin{array}{l}\text { Northwestern University, USA } \\
\text { zelditch@math.northwestern.edu }\end{array}$ \\
\hline
\end{tabular}

PRODUCTION

contact@msp.org

Silvio Levy, Scientific Editor

Sheila Newbery, Senior Production Editor

See inside back cover or pjm.math.berkeley.edu/apde for submission instructions.

The subscription price for 2011 is US \$120/year for the electronic version, and \$180/year for print and electronic. Subscriptions, requests for back issues from the last three years and changes of subscribers address should be sent to Mathematical Sciences Publishers, Department of Mathematics, University of California, Berkeley, CA 94720-3840, USA.

Analysis \& PDE, at Mathematical Sciences Publishers, Department of Mathematics, University of California, Berkeley, CA 94720-3840 is published continuously online. Periodical rate postage paid at Berkeley, CA 94704, and additional mailing offices.

APDE peer review and production are managed by EditFLOW ${ }^{\mathrm{TM}}$ from Mathematical Sciences Publishers.

PUBLISHED BY

mathematical sciences publishers

http://msp.org/

A NON-PROFIT CORPORATION

Typeset in IATEX

Copyright $(2011$ by Mathematical Sciences Publishers 


\section{ANALYSIS \& PDE}

\section{Volume $4 \quad$ No. $3 \quad 2011$}

Regularity of weak solutions of a complex Monge-Ampère equation

GÁBOR SZÉKELYHIDI and VALENTINO TOSATTI

Traveling waves for the cubic Szegó equation on the real line

OANA POCOVNICU

Scattering threshold for the focusing nonlinear Klein-Gordon equation

405

Slim IBRAHIM, NADER MASMOUdi and KenJi NAKANISHI

Rayleigh-type surface quasimodes in general linear elasticity

461

SÖNKE HANSEN 